\title{
A TWO-PHASE SHALLOW DEBRIS FLOW MODEL WITH ENERGY BALANCE
}

\author{
F. Bouchut ${ }^{1}$, E.D. Fernández-Nieto ${ }^{2}$, A. Mangeney ${ }^{3,4}$ \\ AND G. NARBONA-REINA ${ }^{5}$
}

\begin{abstract}
This paper proposes a thin layer depth-averaged two-phase model provided by a dissipative energy balance to describe avalanches of solid-fluid mixtures. This model is derived from a 3D two-phase model based on the equations proposed by Jackson [The Dynamics of Fluidized Particles. Cambridges Monographs on Mechanics (2000)] which takes into account the force of buoyancy and the forces of interaction between the solid and fluid phases. Jackson's model is based on mass and momentum conservation within the two phases, i.e. two vector and two scalar equations. This system has five unknowns: the solid volume fraction, the solid and fluid pressures and the solid and fluid velocities, i.e. three scalars and two vectors. As a result, an additional equation is necessary to close the system. Surprisingly, this issue is inadequately accounted for in the models that have been developed on the basis of Jackson's work. In particular, Pitman and Le [Philos. Trans. R. Soc. A 363 (2005) 799-819] replaced this closure simply by imposing an extra boundary condition. If the pressure is assumed to be hydrostatic, this condition can be considered as a closure condition. However, the corresponding model cannot account for a dissipative energy balance. We propose here a closure equation to complete Jackson's model, imposing incompressibility of the solid phase. We prove that the resulting whole 3D model is compatible with a dissipative energy balance. From this model, we deduce a 2D depth-averaged model and we also prove that the energy balance associated with this model is dissipative. Finally, we propose a numerical scheme to approximate the depth-averaged model. We present several numerical tests for the 1D case that are compared to the results of the model proposed by Pitman and Le.
\end{abstract}

Mathematics Subject Classification. 65C20, 81T80, 91B74, 97M10.

Received April 9, 2013. Revised May 5, 2014.

Published online January 14, 2015.

\footnotetext{
Keywords and phrases. Granular flows, two-phase flows, thin layer approximation, energy balance, non-conservative systems, projection method, finite volume schemes.

1 Université Paris-Est, Laboratoire d'Analyse et de Mathématiques Appliquées (UMR 8050), CNRS, UPEMLV, UPEC, 77454 Marne-la-Vallée, France. francois.bouchut@univ-mlv.fr

2 Departamento de Matemática Aplicada I, Universidad de Sevilla. E.T.S. Arquitectura. Avda, Reina Mercedes, s/n. 41012 Sevilla, Spain. edofer@us.es

3 Université Paris Diderot, Sorbone Paris Cité, Institut de Physique du Globe de Paris, Seismology group, 1 rue Jussieu, 75005 Paris, France. mangeney@ipgp.fr

4 ANGE group INRIA, Jacques Louis Lions, CETMEF

5 Departamento de Matemática Aplicada I, Universidad de Sevilla. E.T.S. Arquitectura. Avda, Reina Mercedes, s/n. 41012

Sevilla, Spain. gnarbona@us.es
} 

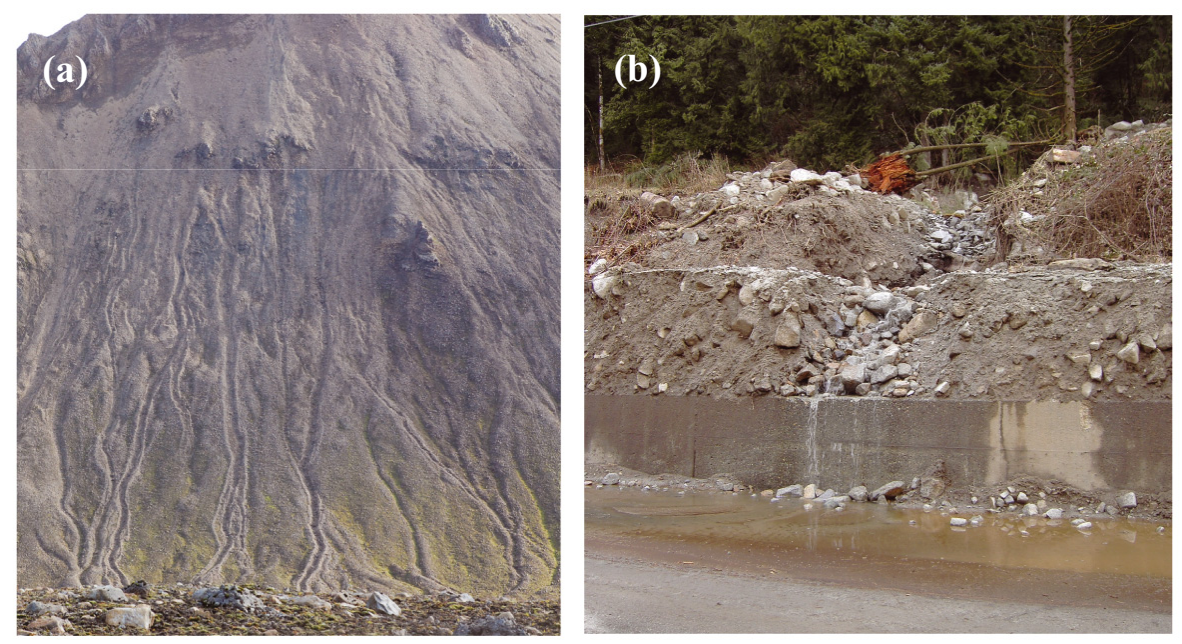

Figure 1. (a) Deposits of several debris flows in Iceland. (b) Close-up of a cross-section of the deposit of a debris flow covering a road in Canada.

\section{INTRODUCTION}

Landslides, debris avalanches or debris flows play a key role in erosion processes on the surface of the Earth and other telluric planets. On Earth, they represent one of the major natural hazards. Gravitational instabilities are also closely related to volcanic, seismic and climatic activity and thus represent potential precursors or proxies for the change of these activities with time. Research involving the dynamic analysis of gravitational mass flows is advancing rapidly. One of its ultimate goals is to produce tools for detection of natural instabilities and for prediction of velocity and runout extent of rapid landslides. The theoretical description and physical understanding of these processes in a natural environment are still open and extremely challenging problems for earth scientists, giving rise to equally challenging mechanical, mathematical and numerical issues. In recent years, significant progress in the mathematical, physical and numerical modelling of gravitational flows has made it possible to develop and use numerical models to investigate geomorphological processes and assess risks related to such natural hazards. However, key questions still remain unanswered, for instance concerning the reason for the high mobility of natural landslides (e.g. [21,22]). Severe limitations prevent a full understanding of physical processes involved in landslide dynamics and the development of tools for detection of instabilities and prediction of their velocity and extent. Indeed, numerical models do not take into account complex natural phenomena such as the static/flowing transition in granular flows or the co-existence and interaction of fluid (water, gas) (e.g. $[7,8,14,18,23,26-28,42,43])$. Water is almost always involved in natural landslides (e.g. [15,16,29]) (Fig. 1b). Interaction forces between the solid and fluid (water) phases may play an important role in flow mobility and deposit extent. Different approaches can be used to simulate fluid-solid mixtures, extending from discrete element models based for example on contact dynamics or molecular dynamics (e.g. [32,47]), and taking into account individual particles, to continuum models that deal with a fluid phase and a solid phase. The discrete element approach is hard to use in geophysical applications due to the high computational costs required to take into account the broad-size distribution of particles in real flows, which is critical in such simulations.

Existing models used to describe the behaviour of fluid-solid mixtures are mainly based on Jackson's model [19]. This model takes into account solid and fluid stresses, the interaction force between the fluid and solid phases and the buoyancy force, through mass and momentum conservation within the two phases. This model thus involves four equations (two scalar and two vector equations). However, the system has five unknowns: the solid volume fraction, the solid and fluid pressures and the solid and fluid velocities (three scalars 
and two vectors). As a result, an additional equation is necessary to close the system. Surprisingly, this issue is inadequately accounted for in the models that have been developed on the basis of Jackson's work.

Solving the 3D two-phase equations leads to high computational costs. For this reason, mostly depth-averaged models have been proposed to deal with natural geophysical flows (e.g. [10,35,36,38]). Iverson [15] was the first to address the need to include interstitial fluid effects in the constitutive behaviour of the mass flow and developed a thin layer model for a solid-fluid mixture moving on realistic terrain, under the simplifying assumptions of constant porosity and equality of the fluid and solid velocity. The flow is described by a single set of equations for the density and momentum of the mixture, which is formally represented by a single-phase model with a stress term accounting for contributions from the two constituents. Due to the lack of an explicit equation for the pore fluid pressure in this model, a pore pressure advection-diffusion equation was added based on experimental measurements. Various versions and applications of this grain-fluid mixture model have since been presented (e.g. Pudasaini et al. [39]; Georges and Iverson, [10]).

Taking another step forward, Pitman and Le proposed in [38] a novel depth-averaged two-fluid model for debris flows, based on Jackson's model, that contains mass and momentum equations for both the fluid and solid phases, thus providing equations for the velocities of the two phases and for solid volume fraction. In the model proposed by Pitman and Le and the modified version proposed by Pelanti et al. [36], the authors do not provide a closure equation for the two-phase model. On the other hand, they impose two boundary conditions involving vanishing surface tension conditions at the free surface, i.e. the pressure of both the solid and the fluid phases vanish at the free surface. Two kinematic boundary conditions are also imposed at the free surface, because the two phases are assumed to fill a common domain, this gives an overdetermined problem at the free surface. However, in the thin layer approximation, because of the hydrostatic pressure assumption, the extra boundary condition makes it possible to express a depth-averaged model, even though no closure relation for the whole system is provided. However, boundary conditions obviously do not replace a closure equation inside the domain. This artificial compensation of the missing closure equation by overdetermined boundary conditions leads to a physically irrelevant energy equation in the Pitman-Le model (see Sect. 4.1).

A physically meaningful energy equation is essential to obtain realistic models. A key issue in two-phase flow models is thus to propose a suitable closure relation that is compatible with the energy balance. Some new and very useful ways to close the system of equations have been proposed by Roux and Radjai [44], Pailha and Pouliquen [35] and George and Iverson [10]. The general idea is to take into account the dilation/compression of the granular phase and its interaction with the pressure of the fluid filling the pores of the granular material. Indeed, these effects have been shown to be crucial at the initiation of mass destabilization and to have a strong impact on the generated flow dynamics [e.g. $[17,40]]$.

Roux and Radjai [44] proposed an equation to describe the evolution of the volume fraction and of the shear stress in a granular material in terms of the shear-induced dilatancy (a property of the granular material related to its dilation when the material is submitted to a shear force). Pailha and Pouliquen used this equation to close their model, based on the two-phase approach proposed by Jackson [35]. They also imposed that both the solid and fluid pressures vanish at the free surface. Moreover, they introduced a closure equation, related to dilatancy effects (Eq. (3.18) in [35]). But, as the resulting system is overdetermined, a condition had to be relaxed. Indeed, they relaxed mass conservation for one of the two phases, that they justified by assuming that the thickness of the flowing mixture is nearly constant.

Alternatively, George and Iverson [10] derived a model using the mass and momentum equations of the mixture. In their model, the unknowns are the total height and velocity of the mixture, the solid volume fraction and the pore fluid pressure. As a closure relation, they used a slightly different equation than that proposed in [44] to describe dilatancy effects, that includes the time derivative of the effective normal stress and the pore fluid pressure. This relation is derived from the mass conservation of the solid phase by assuming that the averaged mixture velocity is equal to the averaged solid velocity (Eqs. (6) and (7) of their paper) and a Darcy law. However, the final model does not impose explicitly the mass conservation of the solid phase.

We propose here to solve the mass and momentum equations of both phases, together with the relevant number of boundary conditions and a closure equation that provides a possibly physically relevant energy 
equation. In a first step toward this objective, we use the simplest closure equation (i.e. incompressibility of the solid phase). We impose a vanishing stress condition at the free surface for the mixture (not for each phase) and kinematic surface boundary conditions (the two phases are supposed to fill the same domain), forming a well-posed 3D system. The analysis of the hydrostatic approximation suggests that a variable related to the pressure field remains in the thin-layer asymptotics. On choosing a static constraint as a closure relation, this extra variable can be determined as the associated Lagrange multiplier. The resulting model has a built-in energy balance.

\section{ThE 3D TWO-PHASE MODEL}

In this section we present the three-dimensional model used to describe the mixture of solid and fluid materials. Note that we do not consider here the role of the air (i.e. a third phase) that can be critical in some cases due to capillary forces, especially at the laboratory scale [16]. As a result, these equations are only valid when the granular media is saturated with fluid so that there is no air within the pores of the granular material. In Section 2.1, the mass and momentum equations of Jackson's model are presented and a closure equation is proposed. In Section 2.2, the boundary conditions are described. In Sections 2.3 and 2.4, we express the drag force and the assumptions concerning the stress tensor. Finally, in Section 2.5, we express the complete model in local coordinates.

\subsection{Mass and momentum equations}

We consider geophysical mass flows made of a mixture of solid and fluid materials. The two fluid model presented below is derived in the Jackson's book [19]. It is based on the dynamics of an assembly of solid particles immersed in a Newtonian fluid. The two-fluid model is obtained by averaging in the whole region the fundamental equations for both components, the fluid and particles. Namely, the Navier-Stokes equation for the motion of the fluid and the equations of linear and angular momentum for each particle for the solid part. These equations are coupled by the no-slip boundary condition imposed on the surface of each particle. The mass and momentum conservation equations for fluid and particle phases are deduced by an averaging procedure. But, some terms linked to the microscopic level of the individual particles are neglected. Consequentely, after the averaging procedure there are more unknowns than equations in the derived system. And a closure for the system must be set.

The two-phase model is defined by the following mass and momentum equations for the solid and fluid phases:

$$
\begin{gathered}
\partial_{t}\left(\rho_{s} \varphi\right)+\nabla \cdot\left(\rho_{s} \varphi v\right)=0, \\
\partial_{t}\left(\rho_{f}(1-\varphi)\right)+\nabla \cdot\left(\rho_{f}(1-\varphi) u\right)=0, \\
\rho_{s} \varphi\left(\partial_{t} v+(v \cdot \nabla) v\right)=-\nabla \cdot T_{s}+f_{0}+\rho_{s} \varphi \mathbf{g}, \\
\rho_{f}(1-\varphi)\left(\partial_{t} u+(u \cdot \nabla) u\right)=-\nabla \cdot T_{f}-f_{0}+\rho_{f}(1-\varphi) \mathbf{g},
\end{gathered}
$$

where the subscript " $s$ " refers to the solid phase and the subscript " $f$ " refers to the fluid phase. The velocities are $v$ for the solid phase and $u$ for the fluid phase. $T$ denotes the stress tensor and $\rho$ the density. Acceleration due to gravity is denoted by $\mathrm{g}$ and $f_{0}$ represents the average value of the resultant force exerted by the fluid on a solid particle. The solid volume fraction is $\varphi$. For monodisperse beads, the maximal volume fraction is $\varphi_{\max } \simeq 0.6$, while it can be higher than 0.9 for highly polydisperse materials because the small particles can fill the pore space between larger particles $([3,13,48])$. The solid fraction is practically never equal to 1 . The case of dry granular flows can be obtained by setting all the variables related to the fluid phase (fluid stress and $f_{0}$ ) 
to zero and volume fraction to one in equations (2.1a) and (2.2a). The minus sign on the stress tensor terms agrees with the sign convention used in soil mechanics, where stress is defined as positive in compression.

Note that both the grain density $\rho_{s}$ and the fluid density $\rho_{f}$ are constant, so that each material is incompressible. However, the density of the solid phase $\varphi \rho_{s}$ (i.e. density of the total amount of grains per unit volume) and the density of the fluid phase $(1-\varphi) \rho_{f}$ (density of the total amount of fluid filling the pores of the granular assembly per unit volume) can change because $\varphi$ varies with space and time. In this sense, the solid and fluid phase could be compressible. Note that the combination of (2.1a) and (2.1b) defines mass conservation for the mixture:

$$
\partial_{t}\left(\rho_{m}\right)+\nabla \cdot\left(\rho_{m} v_{m}\right)=0
$$

where

$$
\rho_{m}=\rho_{s} \varphi+\rho_{f}(1-\varphi) \quad \text { and } \quad v_{m}=\frac{\rho_{s} \varphi v+\rho_{f}(1-\varphi) u}{\rho_{s} \varphi+\rho_{f}(1-\varphi)}
$$

are respectively the density and velocity of the mixture. Multiplying (2.1a) by $\rho_{f}$ and (2.1b) by $\rho_{s}$ gives:

$$
\nabla \cdot(\varphi v+(1-\varphi) u)=0 .
$$

This relation is different from the one expressing incompressibility of the mixture because it does not imply that $\nabla \cdot v_{m}$ is equal to zero.

The averaged value of the interaction force between fluid and particle is collected in $f_{0}$. This force is decomposed into the sum of the buoyancy force $f_{B}$ and all remaining contribution $f$. The main components of this force $f$ are a term depending on the particle concentration and the relative velocity $(u-v)$ - the drag force - , a term depending on the concentration and the relative acceleration - the virtual mass force - and the third contribution due to the force normal to the direction $(u-v)$ - the lift force - .

Several expressions for the buoyancy force are discussed in [19]. In the simplest case this force is written as $f_{B}=-\varphi \nabla p_{f}$ with $p_{f}$ the fluid pressure that resume the force exerted by the fluid at rest on an immersed body that is also at rest. The generalization to more general motions leads us to the expression $f_{B}=-\varphi \nabla T_{f}$, however the approximation of $-\varphi \nabla p_{f}$ gives equivalent equations assuming that the action on the particles due to the gradient of the deviatoric part of $T_{f}$ is collected by the other terms of the total force $f$. So we write

$$
f_{0}=f_{B}+f=-\varphi \nabla p_{f}+f .
$$

In the case when the inertia associated to the relative motion of fluid and particles can be neglected, referred to as the short relaxation time approximation, the virtual mass force may be neglected compared to the drag force in the equations of motion. Regarding to the lift force, the algebraic expression for this contribution normal to the relative velocity is uncertain because it takes quite different forms for different flow regimes. This force will not be considered in this paper. Thus, we assume that $f$ can be expressed simply by the drag force. The drag force acts in the direction of the relative velocity $(u-v)$ and also depends on the particle concentration. So in general it can by written as:

$$
\beta(\varphi,|u-v|)(u-v) .
$$

For small values of $|u-v|$, this force is proportional to the relative velocity so we can write

$$
f=\tilde{\beta}(u-v)
$$

$\tilde{\beta}$ being the drag coefficient (see Sect. 2.3). The notation $\tilde{\beta}$ is used to distinguish this coefficient from the drag coefficients denoted by $\beta$ in some other publications (see for example [35]).

The effective stress tensors $T_{s}$ and $T_{f}$ are related to the interactions between fluid and particles and between particles themselves. In [19] is pointed out the difficulty of writing them in terms of averaged variables in order to close the system. Furthermore an extensive discussion is included to give some explicit and empirical closures for different regimes in terms of the Stokes number. In this work, we take a symmetric solid stress tensor $T_{s}$ and denote $p_{s}$ its first invariant, i.e., the pressure of the solid phase (see Sect. 2.4). 
The viscosity of the fluid acts at the "macroscopic" scale through viscous terms of order $\mu U / L^{2}$, where $U$ and $L$ are characteristic values of respectively the fluid velocity and flow length. On the other hand, the fluid viscosity acts at the "microscopic" scale during the relative motion between the fluid phase and the granular porous media commonly described by the Darcy law. This microscopic contribution is of the order of $\mu \Delta U / \kappa$, where $\kappa$ is the intrinsic hydraulic permeability of the granular media and $\Delta U$ is the typical relative velocity of the fluid phase with respect to the solid phase. Here we assume that the "macroscopic" viscous forces related to the fluid are negligible, so that the fluid stress tensor reduces to the pressure term,

$$
\nabla \cdot T_{f}=\nabla p_{f} .
$$

By substituting these expressions into (2.2a) and (2.2b), we obtain the system (2.1a), (2.1b), and

$$
\begin{aligned}
\rho_{s} \varphi\left(\partial_{t} v+(v \cdot \nabla) v\right) & =-\nabla \cdot T_{s}-\varphi \nabla p_{f}+f+\rho_{s} \varphi \mathbf{g}, \\
\rho_{f}(1-\varphi)\left(\partial_{t} u+(u \cdot \nabla) u\right) & =-(1-\varphi) \nabla p_{f}-f+\rho_{f}(1-\varphi) \mathbf{g} .
\end{aligned}
$$

This system of equations is the same as the system considered in $[16,38]$. Only the boundary conditions are different from those used here.

As discussed above, this system of four equations (2.1a), (2.1b), (2.8a), (2.8b) has five unknowns $\varphi, T_{s}, p_{f}$, $u$ and $v$.

To close the system, we propose to add a supplementary scalar equation, based on the physical processes involved. Starting from the simplest closure relation, we propose to impose the incompressibility of the solid phase:

$$
\nabla \cdot v=0 .
$$

In real granular materials the dilatancy effect may induce changes of the volume of the solid phase, even if the mass of the granular material remains constant. This means that the divergence of the velocity of the solid phase $v$ may not be zero (see [11]). The compression/dilation of the granular phase changes the interstitial fluid pressure that in turn couples with the solid momentum equations. This coupling appears in the non-hydrostatic pressure terms (see [30]), not included in the approximations made in this work.

The consistency of the whole model can be evaluated by the local energy balance equation. To obtain it, we multiply (2.8a), (2.8b) by $v$ and $u$ respectively, combine with (2.1a) and (2.1b), and add the results. This yields

$$
\begin{aligned}
& \partial_{t}\left(\rho_{s} \varphi \frac{|v|^{2}}{2}+\rho_{f}(1-\varphi) \frac{|u|^{2}}{2}\right)+\nabla \cdot\left(\rho_{s} \varphi \frac{|v|^{2}}{2} v+\rho_{f}(1-\varphi) \frac{|u|^{2}}{2} u\right) \\
& =-v \cdot\left(\nabla \cdot T_{s}\right)-(\varphi v+(1-\varphi) u) \cdot \nabla p_{f}+f \cdot(v-u)+\left(\rho_{s} \varphi v+\rho_{f}(1-\varphi) u\right) \cdot \mathbf{g} .
\end{aligned}
$$

Denoting $X$ the space position and once again using (2.1a) and (2.1b) along with (2.4), we obtain

$$
\begin{aligned}
& \partial_{t}\left(\rho_{s} \varphi \frac{|v|^{2}}{2}+\rho_{f}(1-\varphi) \frac{|u|^{2}}{2}-(\mathbf{g} \cdot X)\left(\rho_{s} \varphi+\rho_{f}(1-\varphi)\right)\right) \\
& +\nabla \cdot\left(\rho_{s} \varphi \frac{|v|^{2}}{2} v+\rho_{f}(1-\varphi) \frac{|u|^{2}}{2} u-(\mathbf{g} \cdot X)\left(\rho_{s} \varphi v+\rho_{f}(1-\varphi) u\right)\right. \\
& \left.+p_{f}(\varphi v+(1-\varphi) u)+T_{s} v\right) \\
= & \left(T_{s}-p_{s} \mathrm{Id}\right): \nabla v+p_{s} \nabla \cdot v+f \cdot(v-u),
\end{aligned}
$$

where $p_{s}$ denotes the solid particles pressure.

From equation (2.6), the drag contribution $f \cdot(v-u)$ is non-positive. With the assumption that the solid phase is incompressible, the second term on the right-hand side $p_{s} \nabla \cdot v$ is equal to zero and it is natural to assume that the friction dissipation $\left(T_{s}-p_{s} \mathrm{Id}\right): \nabla v$ is non-positive. As a result, the sum of the three terms in the right-hand side of (2.11) is non-positive. 
The model defined by (2.1a), (2.1b), (2.8a), (2.8b) with closure (2.9) has a locally dissipative energy balance (2.11). Note that in the initial system considered by Pitman and Le, the term $p_{s} \nabla \cdot v$ does not vanish and we cannot ensure the non-positiveness of the right-hand side term in (2.11). We will show in Section 4.1 that the term resulting from the closure equation also makes it possible to obtain a dissipative energy balance in the model.

\subsection{Boundary conditions}

\subsubsection{At the free surface}

We consider the usual geometric setting, which is that the mixture lies in a spatial domain limited by a fixed topography at the bottom and by a free surface at the top.

We assume that the fluid and the solid fill the same domain that is moving with the velocity of both. This gives the simultaneous kinematic conditions

$$
(1, u) \cdot N=0, \quad(1, v) \cdot N=0 \quad \text { at the free surface, }
$$

where $N=\left(N_{t}, N_{X}\right)$ is the time-space normal. It can be rewritten

$$
u \cdot N_{X}=v \cdot N_{X}=-N_{t} \quad \text { at the free surface. }
$$

Note that this is a strong assumption that plays a key role in the derivation of the equations and in the resulting model presented below. In $[16,35,38]$, both the fluid and the solid pressures are set to zero at the free surface. However, as discussed in the introduction, only one dynamic boundary condition can be imposed at the free surface of the mixture:

$$
\left(T_{s}+p_{f} \mathrm{Id}\right) N_{X}=0 \quad \text { at the free surface. }
$$

Remark 2.1 (about the total stress tensor). To obtain the total stress for the mixture we can combine equations $(2.8 \mathrm{a})$ and $(2.8 \mathrm{~b})$. From here the total stress for the mixture becomes more complicated than the sum of the two stress tensors for each phase. Namely, it can be written as $T=T_{s}+T_{f}+T^{\prime}=T_{s}+p_{f} \operatorname{Id}+T^{\prime}$, with $T^{\prime}$ a contribution coming from the non-linear convective terms written through the relative velocities of the solid and the fluid with respect to the velocity of the mixture $v_{m}$ :

$$
T^{\prime}=-\rho_{s} \varphi\left(v-v_{m}\right)\left(v-v_{m}\right)-\rho_{f}(1-\varphi)\left(u-v_{m}\right)\left(u-v_{m}\right) .
$$

Nevertheless, for many geophysical flows one can assume that this term is negligible, by assuming in particular that the relative velocity of the fluid with respect to the solid is small compared to the solid velocity (see p. 540 of [16] for details.). Thus, we can see condition (2.14) as a simplification where the total stress of the mixture at the free surface for our system is defined as the sum of the fluid and solid phase stress tensors.

\subsubsection{At the bottom}

The conditions at the bottom are classically the non-penetration conditions

$$
u \cdot n=0, \quad v \cdot n=0 \quad \text { at the bottom, }
$$

where $n$ is the upward space unit normal (i.e. the normal to the topography).

This must be completed by further conditions for the solid, in particular we consider a Coulomb friction law, following [45]

$$
T_{s} n-\left(\left(T_{s} n\right) \cdot n\right) n=-\tan \delta \operatorname{sign}(v)\left(T_{s} n\right) \cdot n \quad \text { at the bottom, }
$$

where $\delta$ is the intergranular Coulomb friction angle. 
Remark 2.2. The system (2.1a), (2.1b), (2.8a), (2.8b), (2.9) with the boundary conditions (2.13), (2.14), (2.15), (2.16), is formally well-posed. Moreover, we can check that the previous boundary conditions ensure that all the boundary contributions vanish in the energy balance of the model, except the one coming from the Coulomb condition (2.16), which dissipates at the bottom.

The main difference between this system and those considered by Pitman and Le (see [38]) and Pailha and Pouliquen (see [35]) is the definition of the boundary conditions. Instead of considering that the total pressure vanishes at the free surface (Eq. (2.14)), they consider that both the pressure of the solid phase and the pressure of the fluid phase vanish at the free surface. Pitman and Le do not consider any closure equation, consequently we cannot check the well-posedness of this system. Pailha and Pouliquen consider a closure equation in terms of the divergence of the solid phase velocity. Nevertheless, given that the system is overdetermined in this case and they relax the mass conservation of one of the two phases.

\subsection{Assumptions concerning the drag force}

Different empirical relations are proposed in the literature for the drag force. As already mentioned, the drag force expression is assumed to be

$$
f=\tilde{\beta}(u-v) .
$$

The drag coefficient $\tilde{\beta}$ can be defined in different ways:

- Pitman and Le [38] used the drag force proposed by Richardson and Zaki (see [41]):

$$
\tilde{\beta}=\frac{\left(\rho_{s}-\rho_{f}\right) \varphi g}{v_{T}(1-\varphi)^{m-1}},
$$

where $v_{T}$ is the terminal velocity of an isolated representative solid particle falling in the fluid under gravity. This force has been calculated by Richardson and Zaki, based on laboratory experiments measuring $v_{T}$ and $v_{S}$, where $v_{S}$ is the sedimentation velocity of the dispersion of particles in a fluid. Experiments give the empirical law:

$$
v_{S}=(1-\varphi)^{n} v_{T} .
$$

The value of the empirical exponent $n$ lies in the range [2.4, 4.65]. Pitman and Le [38] (Appendix A) show that $m=n-2$, so that $m \in[0.4,2.65]$. Depending on the respective roles of viscous and inertial forces, $v_{S} / v_{T}$ depends or does not depend on the Reynolds number (see [41] for more details).

For example, with the typical values of the experiments done by Iverson [17], the typical Reynolds number is $R_{e}=v_{T} d \rho_{f} / \mu \approx 50$. From Table VI of Richardson and Zacki, this gives $n \approx 3$ and then $m \approx 1$.

- Pailha and Pouliquen [35] use the following definition of the drag coefficient:

$$
\tilde{\beta}=(1-\varphi)^{2} \frac{\mu}{\alpha d^{2}},
$$

$\mu$ being the dynamic viscosity, $d$ the mean grain diameter and

$$
\alpha=\frac{(1-\varphi)^{3}}{150 \varphi^{2}} .
$$

This is derived from the Carman-Kozeny relation for the permeability of the porous media formed by the particles (see $[12,33])$.

Another way to estimate $\tilde{\beta}$ is to assume that the friction between the two phases is similar to the Darcy law. In debris flows, part of the vertical displacements and of the fluctuations of the horizontal displacement are induced by the dilation or the compaction of the granular media. These effects impact on the fluid pressure field that in turn affects the momentum conservation of the solid and fluid phases. The coupling between the fluid and solid phases comes from the drag force (see [35]). This can be understood by considering the deviation 
from the hydrostatic fluid pressure. Let us denote $p_{f}=p_{f}^{h}+p_{f}^{e}$, where $p_{f}^{h}$ corresponds to the hydrostatic fluid pressure, satisfying $\partial_{z} p_{f}^{h}=-\rho_{f} g \cos \theta$, and $p_{f}^{e}$ is the excess pore-fluid pressure. If the right hand side of $(2.8 \mathrm{~b})$ is considered predominant (small inertia), the horizontal variation of $p_{f}^{h}$ is negligible and the gradient of the excess pore-fluid pressure $p_{f}^{e}$ satisfies

$$
\nabla p_{f}^{e}=-\frac{\tilde{\beta}}{(1-\varphi)}(u-v)
$$

This formula has the same structure as the linear Darcian drag formula describing fluid flow within porous media. This law, considered in [10], relates $u-v$ to the gradient of the excess pore-fluid pressure,

$$
\nabla p_{f}^{e}=-\frac{\mu}{\kappa}(1-\varphi)(u-v),
$$

where $\mu$ is the pore-fluid viscosity and $\kappa$ is the intrinsic hydraulic permeability of the granular debris. George and Iverson [10] point out that even if this linear drag formula may oversimplify the effects of complex phaseinteraction forces in debris flows, several research papers, such as $[20,46]$, indicate that it probably provides a suitable first approximation. Comparing (2.20) with the Darcian law (2.21) leads to:

$$
\tilde{\beta}=(1-\varphi)^{2} \frac{\mu}{\kappa},
$$

$\kappa$ being the permeability of the granular media. The value of the effective permeability derived from (2.18) and (2.19), when compared to (2.22) gives respectively:

- For Pitman and Le (2.18):

$$
\kappa=\frac{\mu v_{T}(1-\varphi)^{m+1}}{\left(\rho_{s}-\rho_{f}\right) g \varphi} .
$$

- For Pailha and Pouliquen (2.19):

$$
\kappa=\frac{d^{2}(1-\varphi)^{3}}{150 \varphi^{2}} .
$$

These two different values of permeability derived from (2.23) and (2.24) are compared in Figure 2. For this comparison we set $\mu=0.001 \mathrm{~Pa} \mathrm{~s}, d=10^{-3} \mathrm{~m},\left(\rho_{s}-\rho_{f}\right)=1500 \mathrm{~kg} \mathrm{~m}^{-3}, g=9.81 \mathrm{~m} \mathrm{~s}^{-2}, m=1, v_{T}=$ $0.143 \mathrm{~m} \mathrm{~s}^{-1}$. We observe that both models give relatively close approximations of the permeability for values of $\varphi$ greater than 0.4 .

George and Iverson [10] have simulated the experiments performed in [17]. In these experiments, the value of $\kappa$ was approximately $10^{-12} \mathrm{~m}^{2}$, whereas the numerical simulations where performed with a constant value of $\kappa \approx 10^{-8} \mathrm{~m}^{2}$. Note that, with the definition of $\kappa$ given by (2.23) or $(2.24), \kappa \approx 10^{-8} \mathrm{~m}^{2}$ when $\varphi \approx 0.5$. A value of $\kappa \approx 10^{-12} \mathrm{~m}^{2}$ corresponds to $\varphi \approx 0.9$.

\subsection{Assumptions concerning the stress tensor}

To obtain the final system, a constitutive relation should be stated for the fluid and granular phases.

- Fluid stress tensor $T_{f}$. As mentioned before, we assume that the fluid stress tensor can be expressed by the fluid pressure:

$$
T_{f}^{x y}=T_{f}^{x z}=T_{f}^{y z}=0, \quad T_{f}^{x x}=T_{f}^{y y}=T_{f}^{z z}=p_{f} .
$$

- Solid stress tensor $T_{s}$. We assume that all its components are proportional to the normal stress perpendicular to the topography, i.e. the stress component $T_{s}^{z z}$,

$$
T_{s}^{j k}=\alpha_{j k} T_{s}^{z z}, \quad j, k=x, y, z .
$$




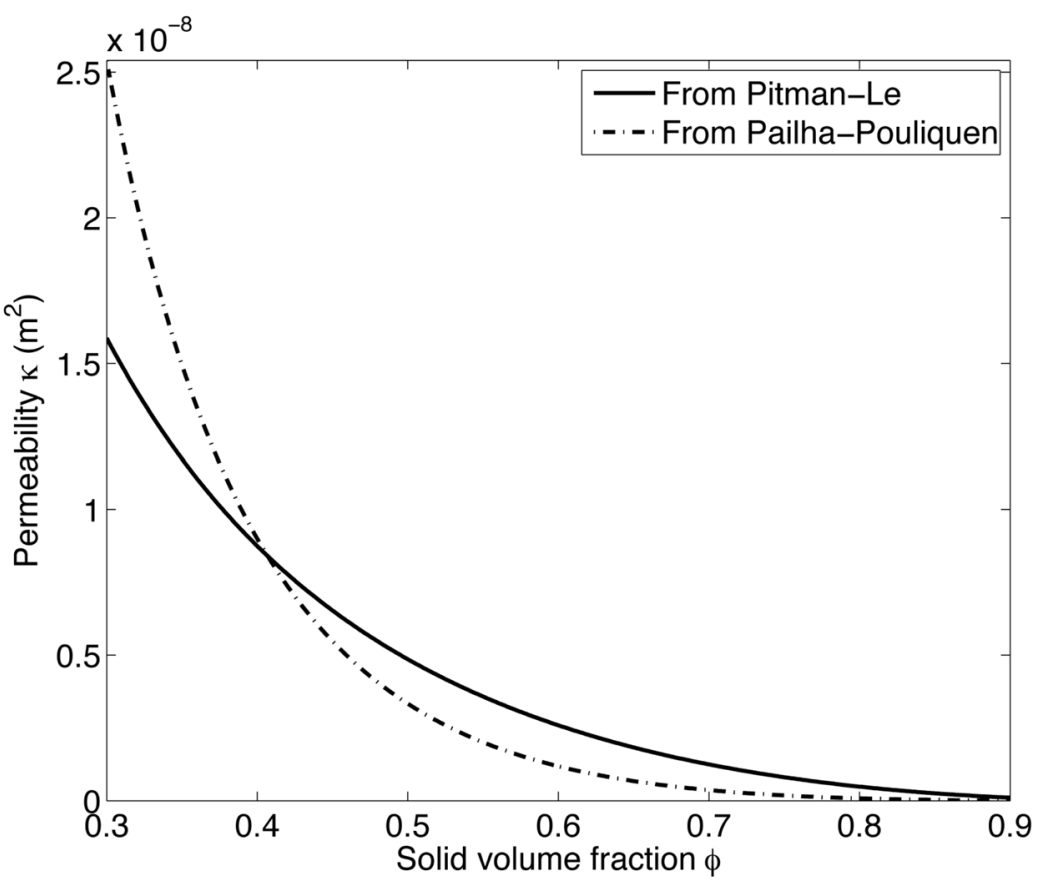

Figure 2. Permeability $\kappa$ as a function of the solid volume fraction $\varphi$. The solid curve corresponds to (2.23) and the dashed curve to (2.24).

The constants $\alpha_{j k}$ are related to the internal and basal Coulomb friction angles, see $[16,38]$ for details. For simplicity, we assume $\alpha_{x x}=\alpha_{y y}=1$, i.e. isotropy of normal stresses $T_{s}^{x x}=T_{s}^{y y}=T_{s}^{z z}$ and we neglect the shear stress in the $x-y$ plane, so that $\alpha_{x y}$ is set to zero.

For the definition of the coefficients $\alpha_{k z}(k=x, y)$, we assume that they satisfy the Mohr-Coulomb plasticity criterion:

$$
\left|\left(T_{s}^{x z}, T_{s}^{y z}\right)\right|=\tan \delta\left|T_{s}^{z z}\right| .
$$

\subsection{Coordinates}

Let us initially write the model in a reference frame related to an inclined plane representative of the mean slope of the topography. More realistic reference frames could be used but such a derivation is beyond the scope of this paper (see for example [5,6]). We consider a fixed slope with constant angle $\theta$ with respect to the horizontal, $-\pi / 2<\theta<\pi / 2$, and the coordinates $(\mathbf{x}, z)$ (assuming $\mathbf{x}=(x, y)$ ) are respectively tangent to and normal to this slope, the $x$ axis being along the steepest direction and the $y$ axis being horizontal. In Figure 3, we represent the coordinates on a cross-section along a vertical plane for clarity. The coordinates of the gravitational force can be written in this reference frame as

$$
\mathbf{g}=(-g \sin \theta, 0,-g \cos \theta)^{t}
$$

We consider a bottom topography $b(\mathbf{x})$ and a thin layer of material over it with thickness $h(t, \mathbf{x})$. The material thus occupies the domain

$$
b(\mathbf{x})<z<b(\mathbf{x})+h(t, \mathbf{x}) .
$$




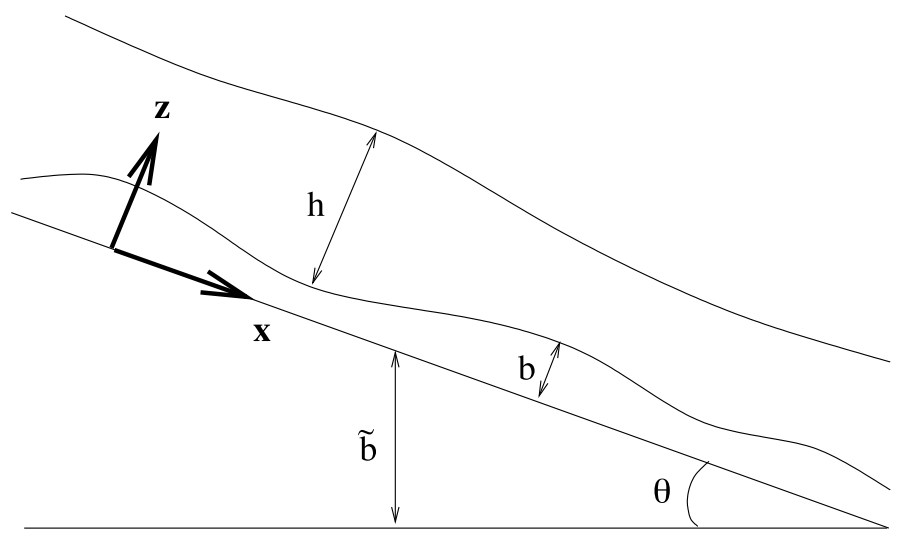

Figure 3. Local coordinates. Here the slope angle $\theta$ is negative.

In this reference frame, the system (2.1a), (2.1b), (2.8a), (2.8b), (2.9) can be written

$$
\begin{gathered}
\partial_{t} \varphi+\nabla_{\mathbf{x}} \cdot\left(\varphi v^{\mathbf{x}}\right)+\partial_{z}\left(\varphi v^{z}\right)=0, \\
\partial_{t}(1-\varphi)+\nabla_{\mathbf{x}} \cdot\left((1-\varphi) u^{\mathbf{x}}\right)+\partial_{z}\left((1-\varphi) u^{z}\right)=0, \\
\rho_{s} \varphi\left(\partial_{t} v^{\mathbf{x}}+v^{\mathbf{x}} \cdot \nabla_{\mathbf{x}} v^{\mathbf{x}}+v^{z} \partial_{z} v^{\mathbf{x}}\right)=-\nabla_{\mathbf{x}} \cdot T_{s}^{\mathbf{x} \mathbf{x}}-\partial_{z} T_{s}^{\mathbf{x} z}-\varphi \nabla_{\mathbf{x}} p_{f}+f_{\mathbf{x}}-g \sin \theta \rho_{s} \varphi(1,0)^{t}, \\
\rho_{s} \varphi\left(\partial_{t} v^{z}+v^{\mathbf{x}} \cdot \nabla_{\mathbf{x}} v^{z}+v^{z} \partial_{z} v^{z}\right)=-\nabla_{\mathbf{x}} \cdot T_{s}^{\mathbf{x} z}-\partial_{z} T_{s}^{z z}-\varphi \partial_{z} p_{f}+f_{z}-g \cos \theta \rho_{s} \varphi, \\
\rho_{f}(1-\varphi)\left(\partial_{t} u^{\mathbf{x}}+u^{\mathbf{x}} \cdot \nabla_{\mathbf{x}} u^{\mathbf{x}}+u^{z} \partial_{z} u^{\mathbf{x}}\right)=-(1-\varphi) \nabla_{\mathbf{x}} p_{f}-f_{\mathbf{x}}-g \sin \theta \rho_{f}(1-\varphi)(1,0)^{t}, \\
\rho_{f}(1-\varphi)\left(\partial_{t} u^{z}+u^{\mathbf{x}} \cdot \nabla_{\mathbf{x}} u^{z}+u^{z} \partial_{z} u^{z}\right)=-(1-\varphi) \partial_{z} p_{f}-f_{z}-g \cos \theta \rho_{f}(1-\varphi), \\
\nabla_{\mathbf{x}} \cdot v^{\mathbf{x}}+\partial_{z} v^{z}=0 .
\end{gathered}
$$

The boundary conditions described in Section 2.2 can be written as follows:

- At the free surface $z=b+h$.

- Free total stress condition

$$
\left(T_{s}+T_{f}\right) N_{X}=0 \quad \text { at } z=b+h,
$$

$N_{X}=\left(-\nabla_{\mathbf{x}}(b+h), 1\right)^{t}$ being the vector normal to the free surface pointing outwards.

- Kinematic condition for each phase

$$
\begin{aligned}
& \partial_{t} h+v^{\mathbf{x}} \cdot \nabla_{\mathbf{x}}(b+h)=v^{z} \quad \text { at } z=b+h, \\
& \partial_{t} h+u^{\mathbf{x}} \cdot \nabla_{\mathbf{x}}(b+h)=u^{z} \quad \text { at } z=b+h,
\end{aligned}
$$

where $u=\left(u^{\mathbf{x}}, u^{z}\right), u^{\mathbf{x}}=\left(u^{x}, u^{y}\right)$ (analogously for $v$ ) and $\nabla_{\mathbf{x}}=\left(\partial_{x}, \partial_{y}\right)$.

- At the bottom $z=b$.

- Non-penetration condition for each phase

$$
\begin{aligned}
& v^{\mathbf{x}} \cdot \nabla_{\mathbf{x}} b=v^{z} \quad \text { at } z=b, \\
& u^{\mathbf{x}} \cdot \nabla_{\mathbf{x}} b=u^{z} \quad \text { at } z=b .
\end{aligned}
$$

- Coulomb friction law

$$
T_{s}^{\mathbf{x} z}=-\tan \delta \operatorname{sign}\left(\mathrm{v}^{\mathbf{x}}\right) \mathrm{T}_{\mathrm{s}}^{\mathrm{zz}} \quad \text { at } \mathrm{z}=\mathrm{b} .
$$




\section{Derivation of the 2D MODEL}

We consider Jackson's system written in inclined coordinates (2.30a) - (2.32b) with closure (2.33), and boundary conditions (2.34)-(2.38). We assume the drag relation (2.17) and consider the stress tensors under the assumptions in Section 2.4. In particular we denote

$$
T_{s}^{z z}=p_{s}, \quad T_{f}=p_{f} \mathrm{Id},
$$

where "Id" is the identity matrix.

$H$ and $L$ are respectively the characteristic width and length of the domain, $T$ a characteristic time and $\epsilon=\frac{H}{L}$, assumed to be small according to the thin layer approximation. We consider the following asymptotic regime in terms of $\epsilon$,

$$
h=\mathcal{O}(\epsilon), \nabla b=\mathcal{O}(\epsilon), \tilde{\beta}=\mathcal{O}(1), T_{s}=\mathcal{O}(\epsilon), p_{f}=\mathcal{O}(\epsilon), u^{\mathbf{x}}=\mathcal{O}(1), v^{\mathbf{x}}=\mathcal{O}(1),
$$

where the reference units are respectively $L, 1, \rho_{f} L / T^{2}, \rho_{s}(L / T)^{2}, \rho_{f}(L / T)^{2}, L / T, L / T$. We shall assume that the unknowns vary at the scales of the domain, which means that formally $\partial_{t}=\mathcal{O}(1), \nabla_{\mathbf{x}}=\mathcal{O}(1)$ and $\partial_{z}=\mathcal{O}(1 / \epsilon)$ with units $1 / T, 1 / L$ and $1 / L$ respectively. For the sake of conciseness, the orders of magnitude will hereafter be expressed without their units. As a first approximation, we also assume that the horizontal velocities, the solid volume fraction $\varphi$ and the friction coefficient $\alpha_{\mathbf{x} z}$ do not depend on $z($ see $[5,6])$,

$$
\begin{aligned}
v^{\mathbf{x}} & =\overline{v^{\mathbf{x}}}(t, \mathbf{x})+\mathcal{O}\left(\epsilon^{2}\right), \\
u^{\mathbf{x}} & =\overline{u^{\mathbf{x}}}(t, \mathbf{x})+\mathcal{O}\left(\epsilon^{2}\right), \\
\varphi & =\bar{\varphi}(t, \mathbf{x})+\mathcal{O}\left(\epsilon^{2}\right), \\
\alpha_{\mathbf{x} z} & =\overline{\alpha_{\mathbf{x} z}}(t, \mathbf{x})+\mathcal{O}\left(\epsilon^{2}\right) .
\end{aligned}
$$

From the divergence condition (2.33), we then obtain that $\partial_{z} v^{z}=-\nabla_{\mathbf{x}} \cdot v^{\mathbf{x}}=\mathcal{O}(1)$, thus integrating with respect to $z$ and using (2.37) we obtain $v^{z}=\mathcal{O}(\epsilon)$. Similarly, the sum of (2.30a) and (2.30b) yields

$$
\nabla_{\mathbf{x}} \cdot\left(\varphi v^{\mathbf{x}}+(1-\varphi) u^{\mathbf{x}}\right)+\partial_{z}\left(\varphi v^{z}+(1-\varphi) u^{z}\right)=0,
$$

giving $\partial_{z}\left(\varphi v^{z}+(1-\varphi) u^{z}\right)=\mathcal{O}(1)$, and with $(2.37)$ and (2.38) we obtain $u^{z}=\mathcal{O}(\epsilon)$.

Note that equations (2.33) and (3.4), with boundary conditions (2.37), (2.38), make it possible to determine $v^{z}, u^{z}$ in terms of $v^{\mathbf{x}}, u^{\mathbf{x}}, \varphi$, without depending on any approximation. Equations (2.31a) and (2.32a) drive the evolution of $v^{\mathrm{x}}, u^{\mathrm{x}}$, and (2.30a) drives the evolution of $\varphi$. Equations (2.31b) and (2.32b) determine $p_{s}$ and $p_{f}$ with boundary condition (2.34). However, there is only one condition (2.34), thus one unknown $p_{s}=-p_{f}$ remains on the free boundary. This extra unknown on the free boundary makes (2.35) and (2.36) solvable. Otherwise, because of the assumption that both phases share the same domain, there would be two equations for only one unknown $h$ on the boundary.

In order to derive the asymptotics, we first focus on the pressure equations. From (2.31b), we obtain

$$
\partial_{z} p_{s}+\bar{\varphi} \partial_{z} p_{f}=-\bar{\varphi} \rho_{s} g \cos \theta+\mathcal{O}(\epsilon),
$$

and from $(2.32 \mathrm{~b})$

$$
(1-\bar{\varphi}) \partial_{z} p_{f}=-(1-\bar{\varphi}) \rho_{f} g \cos \theta+\mathcal{O}(\epsilon)
$$

This yields

$$
\begin{gathered}
p_{f}=p_{f \mid b+h}+\rho_{f} g \cos \theta(b+h-z)+\mathcal{O}\left(\epsilon^{2}\right), \\
p_{s}=p_{s \mid b+h}+\bar{\varphi}\left(\rho_{s}-\rho_{f}\right) g \cos \theta(b+h-z)+\mathcal{O}\left(\epsilon^{2}\right) .
\end{gathered}
$$


However, according to $(2.34),\left(p_{s}+p_{f}\right)_{\mid b+h}=0$. Thus, expressing the previous relations in terms of $p_{f_{\text {bed }}} \equiv$ $p_{f \mid b}=p_{f \mid b+h}+\rho_{f} g h \cos \theta+\mathcal{O}\left(\epsilon^{2}\right)$, we obtain

$$
\begin{gathered}
p_{f}=p_{f_{\mathrm{bed}}}-\rho_{f} g \cos \theta(z-b)+\mathcal{O}\left(\epsilon^{2}\right), \\
p_{s}=-p_{f_{\mathrm{bed}}}+\rho_{f} g h \cos \theta+\bar{\varphi}\left(\rho_{s}-\rho_{f}\right) g \cos \theta(b+h-z)+\mathcal{O}\left(\epsilon^{2}\right) .
\end{gathered}
$$

Note that the unknown $p_{f_{\text {bed }}}$ can be replaced by $p_{s_{\text {bed }}}$ or by $p_{s_{\mid b+h}}=-p_{f_{\mid b+h}}$ via the relations

$$
\begin{aligned}
& p_{f_{\text {bed }}}=-p_{s_{\text {bed }}}+\left(\bar{\varphi} \rho_{s}+(1-\bar{\varphi}) \rho_{f}\right) g h \cos \theta+\mathcal{O}\left(\epsilon^{2}\right), \\
& p_{f_{\text {bed }}}=-p_{s \mid b+h}+\rho_{f} g h \cos \theta+\mathcal{O}\left(\epsilon^{2}\right) .
\end{aligned}
$$

We can then write the tangential components of momentum equations (2.31a) and (2.32a). We assume that

$$
\tilde{\beta}=\bar{\beta}(t, \mathbf{x})+\mathcal{O}\left(\epsilon^{2}\right),
$$

which is the case if $\tilde{\beta}$ is defined by (2.18), with

$$
\bar{\beta}=\frac{\left(\rho_{s}-\rho_{f}\right) \bar{\varphi} g}{v_{T}(1-\bar{\varphi})^{m-1}} .
$$

For the solid phase, this gives

$$
\rho_{s} \bar{\varphi}\left(\partial_{t} \overline{v^{\mathbf{x}}}+\overline{v^{\mathbf{x}}} \cdot \nabla_{\mathbf{x}} \overline{v^{\mathbf{x}}}\right)+\nabla_{\mathbf{x}} p_{s}+\bar{\varphi} \nabla_{\mathbf{x}} p_{f}=-\partial_{z} T_{s}^{\mathbf{x} z}-\bar{\varphi} \rho_{s} g \sin \theta(1,0)^{t}+\bar{\beta}\left(\overline{u^{\mathbf{x}}}-\overline{v^{\mathbf{x}}}\right)+\mathcal{O}\left(\epsilon^{2}\right),
$$

and for the fluid phase

$$
\rho_{f}(1-\bar{\varphi})\left(\partial_{t} \overline{u^{\mathbf{x}}}+\overline{u^{\mathbf{x}}} \cdot \nabla_{\mathbf{x}} \overline{u^{\mathbf{x}}}\right)+(1-\bar{\varphi}) \nabla_{\mathbf{x}} p_{f}=-(1-\bar{\varphi}) \rho_{f} g \sin \theta(1,0)^{t}-\bar{\beta}\left(\overline{u^{\mathbf{x}}}-\overline{v^{\mathbf{x}}}\right)+\mathcal{O}\left(\epsilon^{2}\right) .
$$

To obtain an asymptotic solution to the original system, these two equations must be true for all $z \in(b, b+h)$ and in particular the result must be independent of $z$ up to $\mathcal{O}\left(\epsilon^{2}\right)$, or equivalently its derivative with respect to $z$ must be $\mathcal{O}(\epsilon)$. Taking into account (3.7) and (3.8), we observe that there are two terms that are not independent of $z, \nabla_{\mathbf{x}} p_{s}$ and $\partial_{z} T_{s}^{\mathbf{x} z}$ in (3.13). Since

$$
\begin{aligned}
\nabla_{\mathbf{x}} p_{s}= & -\nabla_{\mathbf{x}} p_{f_{\mathrm{bed}}}+\rho_{f} g \cos \theta \nabla_{\mathbf{x}} h+\bar{\varphi}\left(\rho_{s}-\rho_{f}\right) g \cos \theta \nabla_{\mathbf{x}}(b+h) \\
& +\left(\rho_{s}-\rho_{f}\right) g \cos \theta(b+h-z) \nabla_{\mathbf{x}} \bar{\varphi}+\mathcal{O}\left(\epsilon^{2}\right),
\end{aligned}
$$

the independence with respect to $z$ up to $\mathcal{O}\left(\epsilon^{2}\right)$ in (3.13) is obtained if we suppose

$$
\left(\rho_{s}-\rho_{f}\right) \nabla_{\mathbf{x}} \bar{\varphi}=\mathcal{O}(\epsilon)
$$

and

$$
\partial_{z} T_{s}^{\mathbf{x} z}=\frac{1}{h}\left(T_{s}^{\mathbf{x} z}\left|b+h-T_{s}^{\mathbf{x} z}\right| b \mid+\mathcal{O}\left(\epsilon^{2}\right)\right.
$$

Remark 3.1. Assumptions (3.16) and (3.17) are necessary for the consistency of the asymptotic expansion (3.3), because the $z$ independency in (3.13) justifies that the velocity $v^{\mathbf{x}}$ remains of the form (3.3) for all times. Note that by using (3.27), the assumption (3.16) automatically holds for all times if it does initially.

Nevertheless the model can also be derived without using these two assumptions (3.16) and (3.17), through a classical depth-averaging process (e.g. [38]), or by evaluating the problematic two terms at $z=b+h / 2($ e.g. [5]). For example, if we use the centered second order approximation of the derivative we obtain

$$
\left(\partial_{z} T_{s}^{\mathbf{x} z}\right)_{\mid b+h / 2}=\frac{1}{h}\left(T_{s}^{\mathbf{x} z} \mid b+h-T_{s}^{\mathbf{x} z}{ }_{\mid b}\right)+\mathcal{O}\left(\epsilon^{2}\right)
$$


and the last term in (3.15) becomes

$$
\left(\left(\rho_{s}-\rho_{f}\right) g \cos \theta(b+h-z) \nabla_{\mathbf{x}} \bar{\varphi}\right)_{\mid b+h / 2}=\left(\rho_{s}-\rho_{f}\right) g \cos \theta \frac{h}{2} \nabla_{\mathbf{x}} \bar{\varphi} .
$$

However with this method, the asymptotic expansion of $v^{\mathbf{x}}$ in (3.3) is not well justified. This is why we prefer to state the assumptions (3.16) and (3.17), even if we think that they are not mandatory.

With assumptions (3.16) and (3.17), the last term in (3.15) becomes $\mathcal{O}\left(\epsilon^{2}\right)$, thus negligible. We shall nevertheless keep it under the form of the right-hand side of (3.18), because of the previously discussed possibility of not making assumptions (3.16) and (3.17), and also for getting an energy balance equation, that would not hold without this term.

Taking into account the Coulomb friction law (2.26), (2.39) and (3.3d), we can finally write:

$$
\left(\partial_{z} T_{s}^{\mathbf{x} z}\right)_{\mid b+h / 2}=-\operatorname{sign}\left(\overline{v^{\mathbf{x}}}\right) \tan \delta \frac{1}{h}\left(p_{s \mid b+h}-p_{s \mid b}\right)+\mathcal{O}\left(\epsilon^{2}\right) .
$$

Thus we can write (3.13), (3.14) using (3.7), (3.8), (3.19) and (3.15) evaluated at $z=b+h / 2$, leading to the equations for the horizontal velocities

$$
\begin{aligned}
\rho_{s} \bar{\varphi}\left(\partial_{t} \overline{v^{\mathbf{x}}}+\overline{v^{\mathbf{x}}} \cdot \nabla_{\mathbf{x}} \overline{v^{\mathbf{x}}}\right)= & (1-\bar{\varphi}) \nabla_{\mathbf{x}} p_{f_{\mathrm{bed}}}-(1-\bar{\varphi}) \rho_{f} g \cos \theta \nabla_{\mathbf{x}} h-\bar{\varphi} \rho_{s} g \cos \theta \nabla_{\mathbf{x}}(b+h) \\
& -\left(\rho_{s}-\rho_{f}\right) g \frac{h}{2} \cos \theta \nabla_{\mathbf{x}} \bar{\varphi}-\bar{\varphi} \rho_{s} g \sin \theta(1,0)^{t}+\bar{\beta}\left(\overline{u^{\mathbf{x}}}-\overline{v^{\mathbf{x}}}\right) \\
& -\operatorname{sign}\left(\overline{\mathrm{v}^{\mathbf{x}}}\right) \tan \delta \bar{\varphi}\left(\rho_{\mathrm{s}}-\rho_{\mathrm{f}}\right) g \cos \theta+\mathcal{O}\left(\epsilon^{2}\right) \\
\rho_{f}(1-\bar{\varphi})\left(\partial_{t} \overline{u^{\mathbf{x}}}+\overline{u^{\mathbf{x}}} \cdot \nabla_{\mathbf{x}} \overline{u^{\mathbf{x}}}\right)= & -(1-\bar{\varphi}) \nabla_{\mathbf{x}} p_{f_{\mathrm{bed}}}-(1-\bar{\varphi}) \rho_{f} g \cos \theta \nabla_{\mathbf{x}} b \\
& -(1-\bar{\varphi}) \rho_{f} g \sin \theta(1,0)^{t}-\bar{\beta}\left(\overline{u^{\mathbf{x}}}-\overline{v^{\mathbf{x}}}\right)+\mathcal{O}\left(\epsilon^{2}\right) .
\end{aligned}
$$

Note that the bottom pressure $p_{f_{\text {bed }}}$ is the key additional variable of the model (see Rem. 4.2). We must now deduce equations for $\bar{\varphi}, p_{f_{\text {bed }}}$ and $h$ from (2.30a), (2.35) and (2.36). We first integrate (2.30a) with respect to $z \in(b, b+h)$ and get

$$
\begin{aligned}
\partial_{t} \int_{b}^{b+h} \varphi \mathrm{d} z & +\nabla_{\mathbf{x}} \cdot \int_{b}^{b+h} \varphi v^{\mathbf{x}} \mathrm{d} z \\
& -\varphi_{\mid b+h} \partial_{t} h-\left(\varphi v^{\mathbf{x}}\right)_{\mid b+h} \cdot \nabla_{\mathbf{x}}(b+h)+\left(\varphi v^{\mathbf{x}}\right)_{b} \cdot \nabla_{\mathbf{x}} b+\left(\varphi v^{z}\right)_{\mid b+h}-\left(\varphi v^{z}\right)_{b}=0 .
\end{aligned}
$$

But according to (2.35) and (2.37), the second line vanishes identically. We deduce the transport equation for the solid phase

$$
\partial_{t}(h \bar{\varphi})+\nabla_{\mathbf{x}} \cdot\left(h \bar{\varphi} \overline{v^{\mathbf{x}}}\right)=\mathcal{O}\left(\epsilon^{3}\right) .
$$

A similar computation from the integration of (2.30b) using (2.36) and (2.38) yields the transport equation for the fluid phase

$$
\partial_{t}(h(1-\bar{\varphi}))+\nabla_{\mathbf{x}} \cdot\left(h(1-\bar{\varphi}) \overline{u^{\mathbf{x}}}\right)=\mathcal{O}\left(\epsilon^{3}\right) .
$$

Adding (3.23) and (3.24), we get the transport equation for the mixture,

$$
\partial_{t} h+\nabla_{\mathbf{x}} \cdot\left(h\left(\bar{\varphi} \overline{v^{\mathbf{x}}}+(1-\bar{\varphi}) \overline{u^{\mathbf{x}}}\right)\right)=\mathcal{O}\left(\epsilon^{3}\right) .
$$




\subsection{Closure equation}

We use the closure equation on the incompressibility of the solid velocity (2.33) to write the mass equation for the solid phase $(2.30 \mathrm{a})$ as

$$
\partial_{t} \varphi+v^{\mathbf{x}} \cdot \nabla_{\mathbf{x}} \varphi+v^{z} \partial_{z} \varphi=0
$$

Taking into account the former considerations we get

$$
\partial_{t} \bar{\varphi}+\overline{v^{\mathbf{x}}} \cdot \nabla_{\mathbf{x}} \bar{\varphi}=\mathcal{O}\left(\epsilon^{2}\right) .
$$

Combining this with (3.23) we obtain

$$
\partial_{t} h+\nabla_{\mathbf{x}} \cdot\left(h \overline{v^{\mathbf{x}}}\right)=\mathcal{O}\left(\epsilon^{3}\right) .
$$

Subtracting (3.28) to (3.25) we finally obtain the closure equation

$$
\nabla_{\mathbf{x}} \cdot\left(h(1-\bar{\varphi})\left(\overline{u^{\mathbf{x}}}-\overline{v^{\mathbf{x}}}\right)\right)=\mathcal{O}\left(\epsilon^{3}\right) .
$$

\subsection{Resulting model}

After dropping $\epsilon$, the model is reduced to the following set of equations:

$$
\begin{aligned}
\partial_{t}(h \bar{\varphi})+\nabla_{\mathbf{x}} \cdot\left(h \bar{\varphi} \overline{v^{\mathbf{x}}}\right)= & 0, \\
\partial_{t}(h(1-\bar{\varphi}))+\nabla_{\mathbf{x}} \cdot\left(h(1-\bar{\varphi}) \overline{u^{\mathbf{x}}}\right)= & 0, \\
\rho_{s} \bar{\varphi}\left(\partial_{t} \overline{v^{\mathbf{x}}}+\overline{v^{\mathbf{x}}} \cdot \nabla_{\mathbf{x}} \overline{v^{\mathbf{x}}}\right)= & (1-\bar{\varphi}) \nabla_{\mathbf{x}} p_{f_{\mathrm{bed}}}-(1-\bar{\varphi}) \rho_{f} g \cos \theta \nabla_{\mathbf{x}} h \\
& -\bar{\varphi} \rho_{s} g \cos \theta \nabla_{\mathbf{x}}(b+h)-\frac{1}{2}\left(\rho_{s}-\rho_{f}\right) g h \cos \theta \nabla_{\mathbf{x}} \bar{\varphi} \\
& -\bar{\varphi} \rho_{s} g \sin \theta(1,0)^{t}+\bar{\beta}\left(\overline{u^{\mathbf{x}}}-\overline{v^{\mathbf{x}}}\right) \\
& -\operatorname{sign}\left(\overline{\mathrm{v}^{\mathbf{x}}}\right) \tan \delta \bar{\varphi}\left(\rho_{\mathrm{s}}-\rho_{\mathrm{f}}\right) g \cos \theta, \\
\rho_{f}(1-\bar{\varphi})\left(\partial_{t} \overline{u^{\mathbf{x}}}+\overline{u^{\mathbf{x}}} \cdot \nabla_{\mathbf{x}} \overline{u^{\mathbf{x}}}\right)= & -(1-\bar{\varphi}) \nabla_{\mathbf{x}} p_{f_{\mathrm{bed}}}-(1-\bar{\varphi}) \rho_{f} g \cos \theta \nabla_{\mathbf{x}} b \\
& -(1-\bar{\varphi}) \rho_{f} g \sin \theta(1,0)^{t}-\bar{\beta}\left(\overline{u^{\mathbf{x}}}-\overline{v^{\mathbf{x}}}\right), \\
\nabla_{\mathbf{x}} \cdot\left(h(1-\bar{\varphi})\left(\overline{u^{\mathbf{x}}}-\overline{v^{\mathbf{x}}}\right)\right)= & 0 .
\end{aligned}
$$

In this system, the scalar $p_{f_{\text {bed }}}$ can be viewed as a Lagrange multiplier for the static constraint (3.30e).

\section{Properties of the proposed model}

Consider the model (3.30) in which we omit the "bar" notation for the sake of clarity, and denote the solid and fluid velocities $v$ and $u$ respectively (instead of $v^{\mathbf{x}}$ and $u^{\mathbf{x}}$ ). Thus the system can be expresses as

$$
\begin{aligned}
\partial_{t}(h \varphi)+\operatorname{div}(h \varphi v)= & 0, \\
\partial_{t}(h(1-\varphi))+\operatorname{div}(h(1-\varphi) u)= & 0, \\
\rho_{s} \varphi\left(\partial_{t} v+v \cdot \nabla v\right)= & (1-\varphi) \nabla p_{f_{\text {bed }}}-(1-\varphi) \rho_{f} g \cos \theta \nabla h \\
& -\varphi \rho_{s} g \cos \theta \nabla(b+h)-\frac{1}{2}\left(\rho_{s}-\rho_{f}\right) g h \cos \theta \nabla \varphi \\
& -\varphi \rho_{s} g \sin \theta(1,0)^{t}+\tilde{\beta}(u-v), \\
& -\operatorname{sign}(\mathrm{v}) \tan \delta \varphi\left(\rho_{\mathrm{s}}-\rho_{\mathrm{f}}\right) \mathrm{g} \cos \theta \\
\rho_{f}(1-\varphi)\left(\partial_{t} u+u \cdot \nabla u\right)= & -(1-\varphi) \nabla p_{f_{\mathrm{bed}}}-(1-\varphi) \rho_{f} g \cos \theta \nabla b \\
& -(1-\varphi) \rho_{f} g \sin \theta(1,0)^{t}-\tilde{\beta}(u-v), \\
\operatorname{div}(h(1-\varphi)(u-v))= & 0 .
\end{aligned}
$$


In this section, we will first establish a local energy equation for this model and then describe some of its properties.

\subsection{Local energy}

In the following lines we prove that the model (4.1) is compatible with a dissipative energy balance.

First, from the mass equations (4.1a), (4.1b) we have

$$
\begin{gathered}
\partial_{t} h+\operatorname{div}(h \varphi v+h(1-\varphi) u)=0, \\
\partial_{t}\left(h\left(\rho_{s} \varphi+\rho_{f}(1-\varphi)\right)\right)+\operatorname{div}\left(h\left(\rho_{s} \varphi v+\rho_{f}(1-\varphi) u\right)\right)=0 .
\end{gathered}
$$

We can write $\sin \theta(1,0)^{t}=\cos \theta \nabla \tilde{b}$ with $\tilde{b}=x \tan \theta$, so that the $\sin \theta$ terms in (4.1c) and (4.1d) can be grouped with the $\nabla b$ terms to give $\nabla(b+\tilde{b})$.

Then we multiply equation (4.1c) by $(h v)$ and (4.1d) by $(h u)$ and sum up the results. Using the mass equations to simplify the left-hand side, we obtain

$$
\begin{aligned}
& \partial_{t}\left(\rho_{s} \varphi h \frac{|v|^{2}}{2}+\rho_{f}(1-\varphi) h \frac{|u|^{2}}{2}\right)+\operatorname{div}\left(\rho_{s} \varphi h \frac{|v|^{2}}{2} v+\rho_{f}(1-\varphi) h \frac{|u|^{2}}{2} u\right) \\
= & \underbrace{-(1-\varphi) h(u-v) \cdot \nabla\left(p_{f_{\mathrm{bed}}}-\rho_{f} g h \cos \theta\right)}_{(a)} \underbrace{-g h \cos \theta\left(\rho_{s} \varphi v+\rho_{f}(1-\varphi) u\right) \cdot \nabla(b+\tilde{b}+h)}_{(b)} \\
& \underbrace{-\frac{h^{2}}{2} g \cos \theta\left(\rho_{s}-\rho_{f}\right) v \cdot \nabla \varphi}_{(c)}-\tilde{\beta} h|u-v|^{2}-|v| \tan \delta \varphi\left(\rho_{s}-\rho_{f}\right) g h \cos \theta .
\end{aligned}
$$

Our objective is to compute each term on the right-hand side of the previous equation and try to write it as a time derivative or a divergence of something explicit.

- Term (a). Using (4.1e),

$$
(a)=-\operatorname{div}\left((1-\varphi) h(u-v)\left(p_{f_{\mathrm{bed}}}-\rho_{f} g h \cos \theta\right)\right) .
$$

- Term (b). Taking into account (4.3),

$$
\begin{aligned}
(b)= & -g h \cos \theta \nabla(b+\tilde{b}) \cdot\left(\rho_{s} \varphi v+\rho_{f}(1-\varphi) u\right)-g \cos \theta \nabla\left(\frac{h^{2}}{2}\right) \cdot\left(\rho_{s} \varphi v+\rho_{f}(1-\varphi) u\right) \\
= & -\operatorname{div}\left(g h(b+\tilde{b}) \cos \theta\left(\rho_{s} \varphi v+\rho_{f}(1-\varphi) u\right)\right) \\
& +g(b+\tilde{b}) \cos \theta \operatorname{div}\left(h\left(\rho_{s} \varphi v+\rho_{f}(1-\varphi) u\right)\right) \\
& -\operatorname{div}\left(\frac{1}{2} g h^{2} \cos \theta\left(\rho_{s} \varphi v+\rho_{f}(1-\varphi) u\right)\right)+\frac{1}{2} g h^{2} \cos \theta \operatorname{div}\left(\rho_{s} \varphi v+\rho_{f}(1-\varphi) u\right) \\
= & -\operatorname{div}\left(g h \cos \theta\left(b+\tilde{b}+\frac{h}{2}\right)\left(\rho_{s} \varphi v+\rho_{f}(1-\varphi) u\right)\right) \\
& -\partial_{t}\left(g h(b+\tilde{b}) \cos \theta\left(\rho_{s} \varphi+\rho_{f}(1-\varphi)\right)\right)+\frac{1}{2} g h^{2} \cos \theta \operatorname{div}\left(\rho_{s} \varphi v+\rho_{f}(1-\varphi) u\right) .
\end{aligned}
$$


- Term (c).

$$
(c)=-\operatorname{div}\left(\frac{1}{2} g h^{2} \cos \theta\left(\rho_{s}-\rho_{f}\right) \varphi v\right)+\varphi\left(\rho_{s}-\rho_{f}\right) g \cos \theta \operatorname{div}\left(\frac{h^{2}}{2} v\right) .
$$

Gathering all the terms we get

$$
\begin{aligned}
& \partial_{t}\left(\rho_{s} \varphi h \frac{|v|^{2}}{2}+\rho_{f}(1-\varphi) h \frac{|u|^{2}}{2}+g h(b+\tilde{b}) \cos \theta\left(\rho_{s} \varphi+\rho_{f}(1-\varphi)\right)\right) \\
& \quad+\operatorname{div}\left(\rho_{s} \varphi h \frac{|v|^{2}}{2} v+\rho_{f}(1-\varphi) h \frac{|u|^{2}}{2} u+(1-\varphi) h(u-v)\left(p_{f_{\mathrm{bed}}}-\rho_{f} g h \cos \theta\right)\right. \\
& \left.\quad+g h \cos \theta\left(b+\tilde{b}+\frac{h}{2}\right)\left(\rho_{s} \varphi v+\rho_{f}(1-\varphi) u\right)+\frac{1}{2} g h^{2} \cos \theta\left(\rho_{s}-\rho_{f}\right) \varphi v\right) \\
& =T_{1}
\end{aligned}
$$

where $T_{1}$ can be expressed as

$$
\begin{aligned}
T_{1}= & \frac{1}{2} g h^{2} \cos \theta \operatorname{div}\left(\rho_{s} \varphi v+\rho_{f}(1-\varphi) u\right)+\varphi\left(\rho_{s}-\rho_{f}\right) g \cos \theta \operatorname{div}\left(\frac{h^{2}}{2} v\right) \\
& -\tilde{\beta} h|u-v|^{2}-|v| \tan \delta \varphi\left(\rho_{s}-\rho_{f}\right) g h \cos \theta .
\end{aligned}
$$

The first term can be expressed as

$$
\begin{aligned}
\frac{1}{2} g h^{2} \cos \theta \operatorname{div}\left(\rho_{s} \varphi v+\rho_{f}(1-\varphi) u\right)= & \frac{1}{2} g h \cos \theta \operatorname{div}\left(h\left(\rho_{s} \varphi v+\rho_{f}(1-\varphi) u\right)\right) \\
& -\frac{1}{2} g h \cos \theta\left(\rho_{s} \varphi v+\rho_{f}(1-\varphi) u\right) \cdot \nabla h .
\end{aligned}
$$

However, according to (4.2) and (4.3) we have

$$
\begin{aligned}
\partial_{t}\left(\frac{1}{2} g h^{2} \cos \theta\left(\rho_{s} \varphi+\rho_{f}(1-\varphi)\right)\right)= & -\frac{1}{2} g h \cos \theta \operatorname{div}\left(h\left(\rho_{s} \varphi v+\rho_{f}(1-\varphi) u\right)\right) \\
& -\frac{1}{2} g h \cos \theta\left(\rho_{s} \varphi+\rho_{f}(1-\varphi)\right) \operatorname{div}(h(\varphi v+(1-\varphi) u)),
\end{aligned}
$$

Thus using this in (4.7) we obtain

$$
\begin{aligned}
& \frac{1}{2} g h^{2} \cos \theta \operatorname{div}\left(\rho_{s} \varphi v+\rho_{f}(1-\varphi) u\right) \\
= & -\partial_{t}\left(\frac{1}{2} g h^{2} \cos \theta\left(\rho_{s} \varphi+\rho_{f}(1-\varphi)\right)\right)-\frac{1}{2} g h \cos \theta\left(\rho_{s} \varphi+\rho_{f}(1-\varphi)\right) \operatorname{div}(h v) \\
& -\frac{1}{2} g h \cos \theta\left(\rho_{s} \varphi v+\rho_{f}(1-\varphi) u\right) \cdot \nabla h .
\end{aligned}
$$


Adding the second term in (4.6) yields

$$
\begin{aligned}
& \frac{1}{2} g h^{2} \cos \theta \operatorname{div}\left(\rho_{s} \varphi v+\rho_{f}(1-\varphi) u\right)+\varphi\left(\rho_{s}-\rho_{f}\right) g \cos \theta \operatorname{div}\left(\frac{h^{2}}{2} v\right) \\
= & -\partial_{t}\left(\frac{1}{2} g h^{2} \cos \theta\left(\rho_{s} \varphi+\rho_{f}(1-\varphi)\right)\right)-\frac{1}{2} g h \cos \theta\left(\rho_{s} \varphi+\rho_{f}(1-\varphi)\right) \operatorname{div}(h v) \\
& -\frac{1}{2} g h \cos \theta\left(\rho_{s} \varphi v+\rho_{f}(1-\varphi) u\right) \cdot \nabla h+\varphi\left(\rho_{s}-\rho_{f}\right) g \cos \theta \operatorname{div}\left(\frac{h^{2}}{2} v\right) \\
= & -\partial_{t}\left(\frac{1}{2} g h^{2} \cos \theta\left(\rho_{s} \varphi+\rho_{f}(1-\varphi)\right)\right)-\frac{1}{2} \rho_{f} g h \cos \theta(\operatorname{div}(h v)+(\varphi v+(1-\varphi) u) \cdot \nabla h) .
\end{aligned}
$$

Then we can compute

$$
\begin{aligned}
& -\frac{1}{2} \rho_{f} g h \cos \theta(\operatorname{div}(h v)+(\varphi v+(1-\varphi) u) \cdot \nabla h) \\
= & -\frac{1}{2} \rho_{f} g h \cos \theta \operatorname{div}(h v)-\operatorname{div}\left(\frac{1}{2} \rho_{f} g h^{2} \cos \theta(\varphi v+(1-\varphi) u)\right) \\
& +\frac{1}{2} \rho_{f} g h \cos \theta \operatorname{div}(h(\varphi v+(1-\varphi) u)) \\
= & -\operatorname{div}\left(\frac{1}{2} \rho_{f} g h^{2} \cos \theta(\varphi v+(1-\varphi) u)\right)+\frac{1}{2} \rho_{f} g h \cos \theta \operatorname{div}(h(1-\varphi)(u-v)) .
\end{aligned}
$$

Plugging this into (4.8) and (4.6), we obtain

$$
\begin{aligned}
T_{1}= & -\partial_{t}\left(\frac{1}{2} g h^{2} \cos \theta\left(\rho_{s} \varphi+\rho_{f}(1-\varphi)\right)\right)-\operatorname{div}\left(\frac{1}{2} \rho_{f} g h^{2} \cos \theta(\varphi v+(1-\varphi) u)\right) \\
& -\tilde{\beta} h|u-v|^{2}-|v| \tan \delta \varphi\left(\rho_{s}-\rho_{f}\right) g h \cos \theta .
\end{aligned}
$$

Using this result in (4.5) finally yields the energy identity

$$
\begin{aligned}
& \partial_{t}\left(\rho_{s} \varphi h \frac{|v|^{2}}{2}+\rho_{f}(1-\varphi) h \frac{|u|^{2}}{2}+g h \cos \theta\left(b+\tilde{b}+\frac{h}{2}\right)\left(\rho_{s} \varphi+\rho_{f}(1-\varphi)\right)\right) \\
& +\operatorname{div}\left(\rho_{s} \varphi h \frac{|v|^{2}}{2} v+\rho_{f}(1-\varphi) h \frac{|u|^{2}}{2} u+(1-\varphi) h(u-v) p_{f_{\mathrm{bed}}}-\rho_{f}(1-\varphi) g h^{2} \cos \theta(u-v)\right. \\
& +g h \cos \theta\left(b+\tilde{b}+\frac{h}{2}\right)\left(\rho_{s} \varphi v+\rho_{f}(1-\varphi) u\right)+\frac{1}{2} \varphi g h^{2} \cos \theta\left(\rho_{s}-\rho_{f}\right) v \\
& \left.+\frac{1}{2} \rho_{f} g h^{2} \cos \theta(\varphi v+(1-\varphi) u)\right)=R_{e},
\end{aligned}
$$

with

$$
R_{e}=-\tilde{\beta} h|u-v|^{2}-|v| \tan \delta \varphi\left(\rho_{s}-\rho_{f}\right) g h \cos \theta .
$$

Therefore the model (4.1) has a locally dissipative energy balance, since the residual $R_{e}$ is non-positive. 
Remark 4.1. Identity (4.9) can be obtained (up to $\mathcal{O}\left(\epsilon^{3}\right)$ ) by integration of (2.11) with respect to $z$. This shows that the left-hand side contains the physically relevant energy and energy flux.

Remark 4.2. Let us recall that the Pitman-Le model [38] does not use any closure equation (4.1e). Instead, the Pitman-Le model, in the form proposed by Pelanti et al. [36], can be seen as (4.1) where we set $p_{f_{\text {bed }}}=\rho_{f} g h \cos \theta$ (or equivalently $p_{s \mid b+h}=0$ according to (3.10)). Consequently, the energy equation satisfied by the Pitman-Le model is (4.9) with a right-hand side $R_{e}$ that is not always non-positive. The residual term for the Pitman-Le model is

$$
R_{e}=-\frac{1}{2} \varphi\left(\rho_{s}-\rho_{f}\right) g h \cos \theta \operatorname{div}(h(1-\varphi)(u-v))-\tilde{\beta} h|u-v|^{2}-|v| \tan \delta \varphi\left(\rho_{s}-\rho_{f}\right) g h \cos \theta
$$

and has no fixed sign (we will study this term in Test 1 presented in Sect. 5.2.1). The intrinsic reason why the Pitman-Le model has a physically irrelevant energy equation is that it is derived from a 3D model that does not have an energy dissipation principle (see Eq. (2.11)).

\subsection{Other properties}

Model (4.1) is a balance law type system with non-local terms related to $p_{f_{\text {bed }}}$. Note first that it is possible to eliminate $p_{f_{\text {bed }}}$ from the system. Indeed, $p_{f_{\text {bed }}}$ appears only in $(4.1 \mathrm{c})$ and $(4.1 \mathrm{~d})$. We can thus retain the sum of (4.1c) and (4.1d) and if we express $\nabla p_{f_{\text {bed }}}$ from (4.1c) for example and write that the curl of the result vanishes, we obtain the missing relation.

Proposition 4.3. System (4.1) has the following properties.

(i) The two mass equations are conservative. The momentum equations take the quasi-conservative form

$$
\begin{aligned}
\rho_{s}\left(\partial_{t}(h \varphi v)+\operatorname{div}(h \varphi v \otimes v)\right)= & h\left((1-\varphi) \nabla p_{f_{\mathrm{bed}}}-(1-\varphi) \rho_{f} g \cos \theta \nabla h\right. \\
& -\varphi \rho_{s} g \cos \theta \nabla(b+h)-\frac{1}{2}\left(\rho_{s}-\rho_{f}\right) g h \cos \theta \nabla \varphi \\
& -\varphi \rho_{s} g \sin \theta(1,0)^{t}+\tilde{\beta}(u-v) \\
& \left.-\operatorname{sign}(\mathrm{v}) \tan \delta \varphi\left(\rho_{\mathrm{s}}-\rho_{\mathrm{f}}\right) \mathrm{g} \cos \theta\right), \\
& \\
\rho_{f}\left(\partial_{t}(h(1-\varphi) u)+\operatorname{div}(h(1-\varphi) u \otimes u)\right)= & h\left(-(1-\varphi) \nabla p_{f_{\mathrm{bed}}}-(1-\varphi) \rho_{f} g \cos \theta \nabla b\right. \\
& \left.-(1-\varphi) \rho_{f} g \sin \theta(1,0)^{t}-\tilde{\beta}(u-v)\right) .
\end{aligned}
$$

The total momentum takes the conservative form

$$
\begin{aligned}
& \rho_{s}\left(\partial_{t}(h \varphi v)+\operatorname{div}(h \varphi v \otimes v)\right)+\rho_{f}\left(\partial_{t}(h(1-\varphi) u)+\operatorname{div}(h(1-\varphi) u \otimes u)\right) \\
& =-\nabla\left(\left(\rho_{s} \varphi+\rho_{f}(1-\varphi)\right) g \frac{h^{2}}{2} \cos \theta\right)-g \cos \theta\left(\rho_{s} \varphi+\rho_{f}(1-\varphi)\right) h \nabla(b+\tilde{b}) \\
& \quad-\operatorname{sign}(\mathrm{v}) \tan \delta \varphi\left(\rho_{\mathrm{s}}-\rho_{\mathrm{f}}\right) \operatorname{gh} \cos \theta .
\end{aligned}
$$

(ii) The thickness $h$ remains non-negative, and $0 \leq \varphi \leq 1$. 
(iii) Special solutions are the steady states at rest, characterized by

$$
u=v=0, \quad b+\tilde{b}+h=C s t, \quad \varphi=C s t,
$$

where we recall that $\tilde{b}=x \tan \theta$. Indeed this is a solution to our system with $p_{f_{\mathrm{bed}}}=\rho_{f} g h \cos \theta$.

(iv) The classical single fluid shallow water system is obtained when $u=v, \rho_{f}=\rho_{s}=\rho$ and $p_{f_{\mathrm{bed}}}=\rho g h \cos \theta$, and either $\varphi=1$ in equation (4.11a) or $\varphi=0$ in equation (4.11b).

\section{NumERICAL APPROXIMATION}

In this section we describe a numerical method to approximate the proposed two-phase model (4.1) in one dimension. Then we perform different tests, including a comparison with the solution provided by the Pitman-Le model.

We focus on the one-dimensional situation. As pointed out previously, the model can be rewritten in terms of the solid pressure at the free surface $p_{s \mid b+h}$, the fluid pressure at the free surface $p_{f \mid b+h}=-p_{s \mid b+h}$ or the solid pressure at the bed $p_{s \text { bed }}$, instead of the fluid pressure at the bed $p_{f_{\text {bed }}}$, via relations (3.9) and (3.10). In this section we consider the formulation in terms of the solid pressure at the free surface $p_{s \mid b+h}$, which can be written

$$
\begin{aligned}
\partial_{t}(h \varphi)+\partial_{x}(h \varphi v)= & 0, \\
\partial_{t}(h(1-\varphi))+\partial_{x}(h(1-\varphi) u)= & 0, \\
\partial_{t}(h \varphi v)+\partial_{x}\left(h \varphi v^{2}\right)= & -h(1-\varphi) \partial_{x} \psi-\varphi g h \cos \theta \partial_{x}(b+h) \\
& -\frac{1}{2}(1-r) g h^{2} \cos \theta \partial_{x} \varphi \\
& -\varphi g h \sin \theta+\hat{\beta} h(u-v), \\
& -\operatorname{sign}(\mathrm{v}) \tan \delta \mathrm{g} \cos \theta(1-\mathrm{r}) \mathrm{h} \varphi, \\
\partial_{t}(h(1-\varphi) u)+\partial_{x}\left(h(1-\varphi) u^{2}\right)= & \frac{h}{r}(1-\varphi) \partial_{x} \psi-(1-\varphi) g h \cos \theta \partial_{x}(b+h) \\
& -(1-\varphi) g h \sin \theta-\frac{1}{r} \tilde{\beta} h(u-v), \\
\partial_{x}(h(1-\varphi)(u-v))= & 0,
\end{aligned}
$$

where

$$
r=\rho_{f} / \rho_{s}, \quad \hat{\beta}=\frac{\tilde{\beta}}{\rho_{s}}, \quad \psi=\frac{p_{s_{\mid b+h}}}{\rho_{s}}=\frac{\rho_{f} g h \cos \theta-p_{f_{\mathrm{bed}}}}{\rho_{s}} .
$$

If we consider that the drag coefficient $\tilde{\beta}$ is defined by (3.12), then

$$
\hat{\beta}=\frac{(1-r) \varphi g}{v_{T}(1-\varphi)^{m-1}} .
$$

\subsection{Numerical method}

We apply a splitting algorithm, similar to the Teman-Chorin method for incompressible Euler equations, in order to impose the constraint (5.1e). We observe that at the first step, when we neglect the extra unknown $\psi$ (which can be seen as a Lagrange multiplier associated with the constraint) in (5.1a)-(5.1d), we obtain the Pitman-Le model in the form proposed in [36], which is hyperbolic whenever $u-v$ is not too large.

We consider the space domain $[0, L]$ divided in cells $I_{i}=\left(x_{i-1 / 2}, x_{i+1 / 2}\right)$. For simplicity, we assume that these cells have a constant size $\Delta x$. We define $x_{i+\frac{1}{2}}=i \Delta x$ and $x_{i}=(i-1 / 2) \Delta x$, the center of the cell $I_{i}$. Let $\Delta t$ be the time step and define $t^{n+1}=t^{n}+\Delta t . W$ is the vector of the following unknowns of the problem,

$$
W=[h \varphi, h(1-\varphi), h \varphi v, h(1-\varphi) u] .
$$


Therefore $W_{i}^{n}$ denotes the approximation provided by the numerical scheme of the cell averages of the solution,

$$
W_{i}^{n} \cong \frac{1}{\Delta x} \int_{x_{i-1 / 2}}^{x_{i+1 / 2}} W\left(t^{n}, x\right) \mathrm{d} x
$$

and by $\psi_{i+1 / 2}^{n}$, an approximation of $\psi\left(t^{n}, x_{i+1 / 2}\right)$.

Assuming that the values of $W_{i}^{n}$ are known, the system can be discretized in two steps.

- First step. We compute the state $W^{*}=\left[h^{*} \varphi^{*}, h^{*}\left(1-\varphi^{*}\right), h^{*} \varphi^{*} v^{*}, h^{*}\left(1-\varphi^{*}\right) u^{*}\right]$ by a semi-implicit discretization for the drag

$$
\begin{aligned}
W_{i}^{*}= & W_{i}^{n}-\frac{\Delta t}{\Delta x} \mathcal{L}\left(W_{i-1}^{n}, W_{i}^{n}, W_{i+1}^{n}, \Delta(b+\tilde{b})_{i-1 / 2}, \Delta(b+\tilde{b})_{i+1 / 2}\right) \\
& +\left(0,0, \Delta t \hat{\beta}_{i}^{*} h_{i}^{*}\left(u_{i}^{*}-v_{i}^{*}\right),-\Delta t \hat{\beta}_{i}^{*} \frac{h_{i}^{*}}{r}\left(u_{i}^{*}-v_{i}^{*}\right)\right)
\end{aligned}
$$

where $\mathcal{L}\left(W_{i-1}^{n}, W_{i}^{n}, W_{i+1}^{n}, \Delta(b+\tilde{b})_{i-1 / 2}, \Delta(b+\tilde{b})_{i+1 / 2}\right)$ defines the space discretization operator applied to model (5.1a) $-(5.1 \mathrm{~d})$ with $\psi=0$ and $\hat{\beta}=0$. In this work, we have considered the generalized Roe method proposed in [34]. Another possibility would be to use the relaxation solver proposed by Pelanti et al. [37].

- Second step. In order to enforce the constraint, we set $h_{i}^{n+1}=h_{i}^{*}$ and $\varphi_{i}^{n+1}=\varphi_{i}^{*}$ and $v_{i}^{n+1}, u_{i}^{n+1}$ and $\psi_{i+1 / 2}^{n+1}$ are solutions to the following coupled system,

$$
\left\{\begin{array}{l}
(h \varphi v)_{i}^{n+1}=(h \varphi v)_{i}^{*}-\frac{\Delta t}{\Delta x}\left(1-\varphi_{i}^{*}\right) h_{i}^{*}\left(\psi_{i+1 / 2}^{n+1}-\psi_{i-1 / 2}^{n+1}\right), \\
(h(1-\varphi) u)_{i}^{n+1}=(h(1-\varphi) u)_{i}^{*}+\frac{\Delta t}{\Delta x}\left(1-\varphi_{i}^{*}\right) \frac{h_{i}^{*}}{r}\left(\psi_{i+1 / 2}^{n+1}-\psi_{i-1 / 2}^{n+1}\right), \\
(h(1-\varphi)(u-v))_{i+1}^{n+1}-(h(1-\varphi)(u-v))_{i}^{n+1}=0 .
\end{array}\right.
$$

By extracting $v_{i}^{n+1}$ and $u_{i}^{n+1}$ from the two first equations of (5.7) and by substitution in the third equation, we obtain the following system with unknowns $\left\{\psi_{i+1 / 2}^{n+1}\right\}_{i}$,

$$
-a_{i+1}^{*} \psi_{i+3 / 2}^{n+1}+\left(a_{i}^{*}+a_{i+1}^{*}\right) \psi_{i+1 / 2}^{n+1}-a_{i}^{*} \psi_{i-1 / 2}^{n+1}=(h(1-\varphi)(u-v))_{i+1}^{*}-(h(1-\varphi)(u-v))_{i}^{*},
$$

with

$$
a_{i}^{*}=\frac{\Delta t}{\Delta x} h_{i}^{*}\left(1-\varphi_{i}^{*}\right)\left(\frac{1}{r}+\frac{1-\varphi_{i}^{*}}{\varphi_{i}^{*}}\right) .
$$

Thus, in this second step, we must solve system (5.8) (with Dirichlet boundary conditions, $\psi=0$ ) to obtain $\left\{\psi_{i+1 / 2}^{n+1}\right\}_{i}$ and use these values to update $v_{i}^{n+1}$ and $u_{i}^{n+1}$ by the two first equations of (5.7).

The obtained scheme is obviously well-balanced with respect to the steady states at rest (4.13) if the hyperbolic solver $\mathcal{L}$ is well-balanced, and preserves the natural bounds $h \geq 0$ and $0 \leq \varphi \leq 1$.

\subsection{Numerical tests}

We will now present some numerical tests in order to compare the solution of the proposed model (5.1a) - (5.1e) with the solution of the modified Pitman and Le problem proposed in [36] (with the same drag coefficient $\tilde{\beta}$ ).

We simulate the collapse of a column made of a mixture of grains and water first over a horizontal plane and then over an inclined plane, a situation widely investigated for dry granular flows (see for example $[8,25,27]$ ). First, we simulate the flow of the mixture over a horizontal bed for the two different drag forces given by $(2.18)$ and (2.19). In the second test, we simulate the flow of the mixture over an inclined bed of constant slope for a fixed choice of these parameters. 
As general considerations, we fix the CFL number as 0.8 , acceleration due to gravity $g=9.81 \mathrm{~m} \mathrm{~s}^{-2}$ and the material densities $\rho_{f}=1000 \mathrm{~kg} \mathrm{~m}^{-3}$ and $\rho_{s}=2500 \mathrm{~kg} \mathrm{~m}^{-3}$, respectively. Therefore the ratio of densities is $r=0.4$.

\subsubsection{Test 1: Flat bottom}

In this experiment, the space domain is $\Omega=[0,10] \mathrm{m}$ and we consider 200 points. At time $t=5 \mathrm{~s}$, the solid phase is stopped and some small velocities appear in the fluid phase. The initial conditions are defined as follows

$$
h(t=0 \mathrm{~s})=\left\{\begin{array}{l}
0.5 \mathrm{~m} 4 \mathrm{~m} \leq x \leq 6 \mathrm{~m} \\
0.1 \mathrm{~m} \text { otherwise }
\end{array} ; \quad u(t=0 \mathrm{~s})=v(t=0 \mathrm{~s})=0 \mathrm{~m} \mathrm{~s}^{-1} ; \quad \varphi(t=0 \mathrm{~s})=\varphi_{0}\right.
$$

For the initial solid volume fraction, we consider two values of $\varphi_{0}(0.3$ and 0.6$)$ to see the effect the initial state of mixture fluidization. We consider the intergranular Coulomb angle to be $\delta=18^{\circ}$. The objective of this test is to check the influence of the drag force and initial solid volume fraction on the flow and deposit. Remember that the drag friction laws used here (see Sect. 2.3) are given by:

$$
f=\tilde{\beta}(u-v),
$$

where the drag coefficient $\tilde{\beta}$ can be set according to:

- Richardson and Zaki [41]:

$$
\tilde{\beta}=\frac{\left(\rho_{s}-\rho_{f}\right) \varphi g}{v_{T}(1-\varphi)^{m-1}}, \quad \text { with } \quad m \in[0.4,2.65] .
$$

- Pailha and Pouliquen [35]:

$$
\tilde{\beta}=\frac{150 \mu \varphi^{2}}{d^{2}(1-\varphi)} .
$$

We also set

$$
v_{T}=0.143 \mathrm{~m} \mathrm{~s}^{-1}, \mu=10^{-3} \mathrm{~Pa} \mathrm{~s}, d=10^{-3} \mathrm{~m}
$$

and we vary the coefficient $m$, using the values

$$
m=0.4,1 \text { and } 2.65 \text {. }
$$

In the following, we will refer to "RZ" and "PP" for the Richardson and Zaki and for the Pailha and Poliquen drag forces respectively.

\section{Influence of the drag force.}

Figures 4 and 5 show the thickness of the mass (i.e. at time $t=5 \mathrm{~s}$ ) and the associated volume fraction simulated with different drag forces both with the Pitman-Le (PL) model and with the new model proposed here. At that time, the solid phase has completely stopped. In Figure 4 we also compare these two-phase flow models with the results obtained with the Savage-Hutter model, where the fluid phase is not considered (i.e. dry granular flows). Even for $\varphi_{0}=0.6$, Figure 4 show the strong influence of the fluid phase on the avalanche thickness profile.

The first observation is that the PL model and our model have the same qualitative behaviour. However, for $\varphi_{0}=0.3$, the PL model is more sensitive to the different drag forces introduced in the model (Figs. 4a and 5a). In particular, the final volume fraction is higher for the PP drag force than for the RZ drag force at a centered mass interval, $x \in(2 m, 8 m)$. They coincides just in the center of mass $(x=5 m)$ for the case $m=0.4$, reaching up to $\varphi=0.65$. For the RZ drag forces, the variation of the volume fraction in space is smoother than for the PP drag force. For our model, the drag force only slightly affects the results for $\varphi_{0}=0.3$. For $\varphi_{0}=0.6$, the sensitivity of the two models to the different drag forces is qualitatively similar even though the volume fraction calculated with the PL model is still more sensitive than that calculated with our model (Figs. 5b,d). For $\varphi_{0}=0.6$, the volume fraction at the center of the column reaches very high values with the PL model 


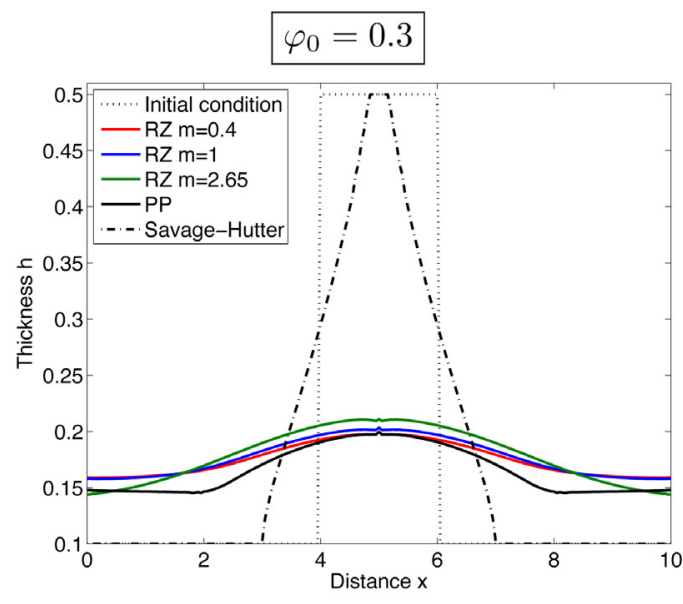

(a) $h(t=5 \mathrm{~s})$. Pitman-Le model.

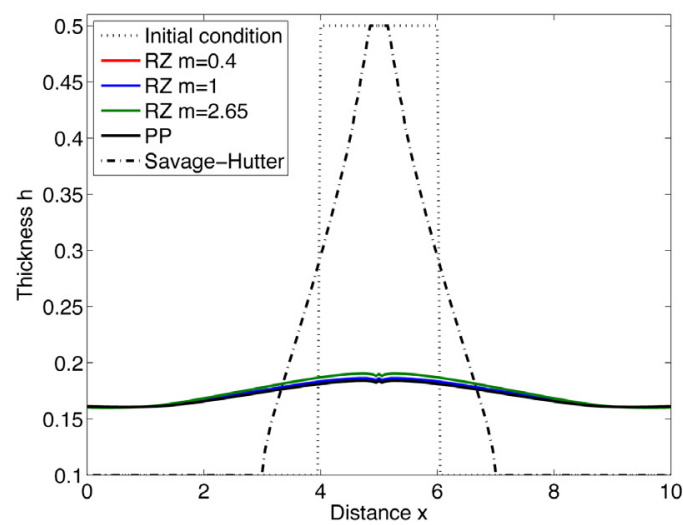

(c) $h(t=5 \mathrm{~s})$. Proposed model.

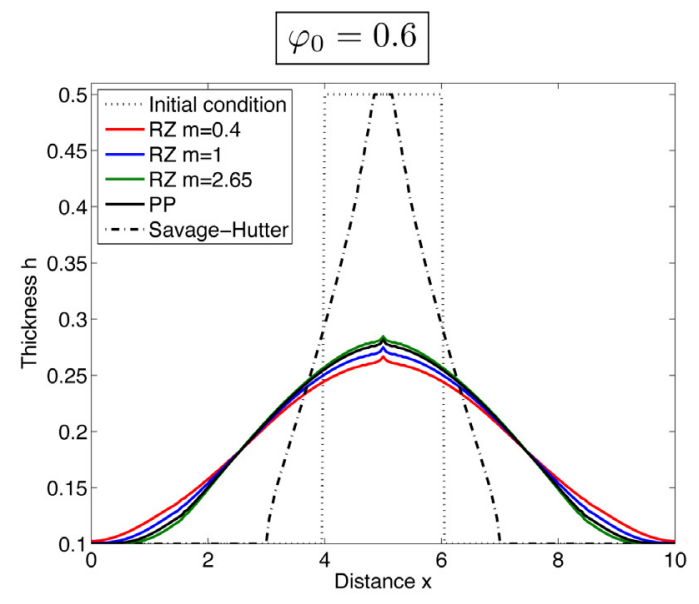

(b) $h(t=5 \mathrm{~s})$. Pitman-Le model.

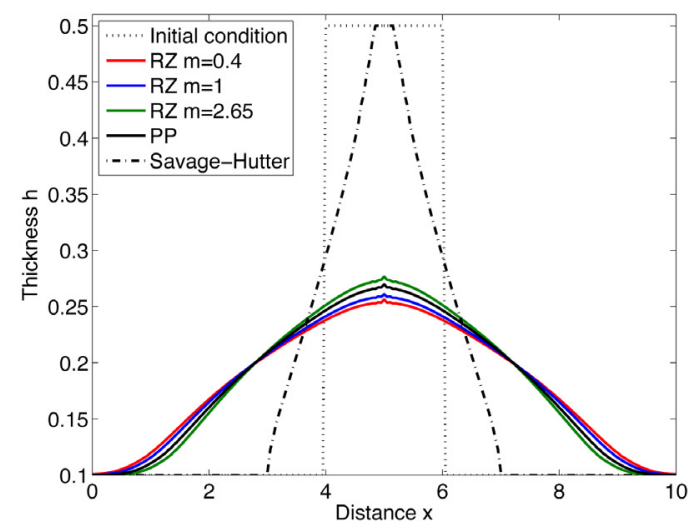

(d) $h(t=5 \mathrm{~s})$. Proposed model.

Figure 4. Test 1: thickness profile of the mass $h(\mathrm{~m})$ as a function of the distance $x(\mathrm{~m})$ at time $t=5 \mathrm{~s}$, when the granular phase has already stopped, for the collapse of a rectangular granular mixture over a horizontal layer made of the same mixture, simulated with different friction laws ("RZ" refers to the Richardson and Zaki and "PP" to the Pailha and Pouliquen drag forces). The initial volume fractions are: (left) $\varphi_{0}=0.3$; (right) $\varphi_{0}=0.6$. The thickness profile obtained using the dry granular flow model of Savage and Hutter (obtained by setting all the terms related to the fluid phase equal to zero) is also represented for comparison.

(about 0.9 ), while $\varphi<0.75$ with our model. The overall lower sensitivity of our model to the different drag forces suggests that the difference between the velocities of the two phases is lower with our model than with the PL model, as shown in Figure 8.

\section{Comparison of the two models.}

Let us now compare the two models for a given friction law, i.e., the Richardson and Zaki law with $m=1$ (corresponding to the data used in [10]). We also consider two different values for the Coulomb friction angle: $\delta=18^{\circ}$ and $\delta=28^{\circ}$.

For both the PL model and our model, the higher the initial fluidization, the larger the spreading of the material and the smaller the aspect ratio of the deposit (Fig. 6). Furthermore, the volume fraction is highest at the center of mass and decreases toward the front, leading to $\varphi<\varphi_{0}$ at the front. 


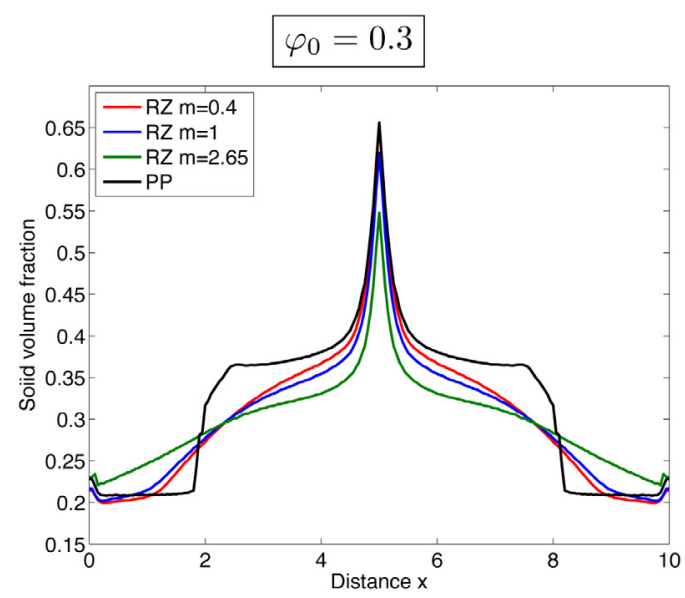

(a) $\varphi(t=5 \mathrm{~s})$. Pitman-Le model.

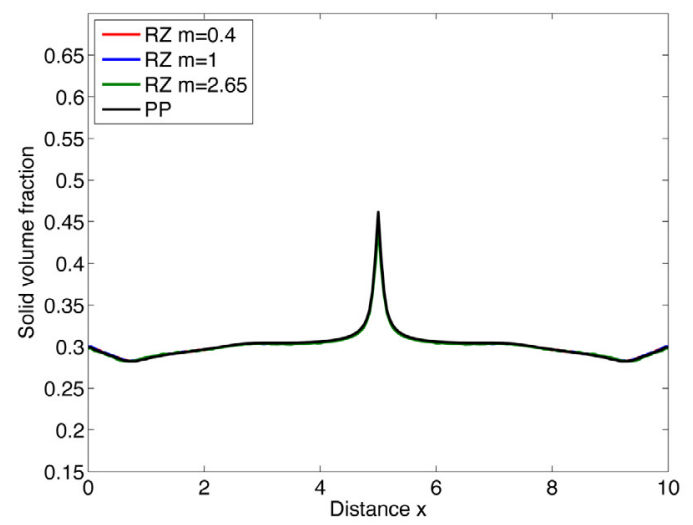

(c) $\varphi(t=5 \mathrm{~s})$. Proposed model.

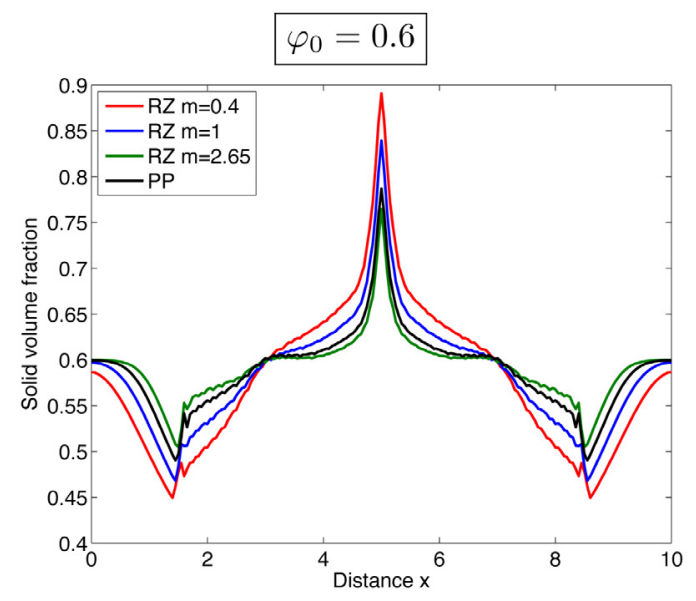

(b) $\varphi(t=5 \mathrm{~s})$. Pitman-Le model.

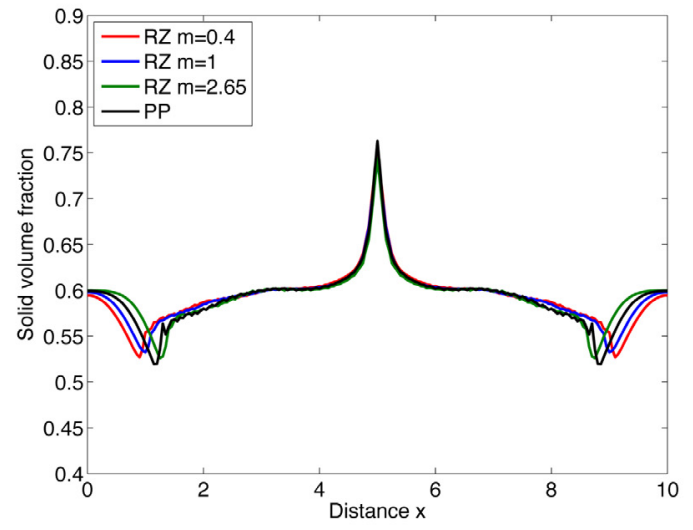

(d) $\varphi(t=5 \mathrm{~s})$. Proposed model.

Figure 5. Test 1: solid volume fraction of the mass $\varphi$ as a function of the distance $x(\mathrm{~m})$ at time $t=5 \mathrm{~s}$, when the granular phase has already stopped, for the collapse of a rectangular granular mixture over a horizontal layer made of the same mixture, simulated with different friction laws ("RZ" refers to the Richardson and Zaki and "PP" to the Pailha and Pouliquen drag forces). The initial volume fractions are: (left) $\varphi_{0}=0.3$; (right) $\varphi_{0}=0.6$.

At time $t=5 \mathrm{~s}$, when the solid phase is already at rest, the thickness of the mass is very similar in both models, even though the maximum thickness is slightly smaller with our model (Fig. 6). However, the distribution of the phases (i.e. volume fraction) is different. As observed previously, the volume fraction is more uniformly distributed in the simulations with our model. The peak of high volume fraction at the center of mass is higher for the PL model and the decrease in volume fraction toward the front of the mass is larger than in our model (Fig. 6c).

The velocity of the fluid phase at an intermediate time $t=1.5 \mathrm{~s}$, when the granular phase is still flowing, is slightly higher with our model for $\delta=18^{\circ}$ for both $\varphi_{0}=0.3$ and $\varphi_{0}=0.6$ (see Figs. $7 \mathrm{a}$ and $7 \mathrm{~b}$ ). For $\delta=28^{\circ}$, the fluid velocities are almost the same in the two models towards the front and the fluid velocity with our model is lower around the center than that calculated with the PL model. The difference between the two models is greater for the solid velocity for both $\varphi_{0}=0.3$ and $\varphi_{0}=0.6$, (see Figs. 7c and 7d). For the proposed model, the solid phase moves faster than for the PL model. We also observe that for $\delta=18^{\circ}$, the velocities of both phases are greater than for $\delta=28^{\circ}$. Moreover, for larger values of $\delta$, the difference between the velocities of the 


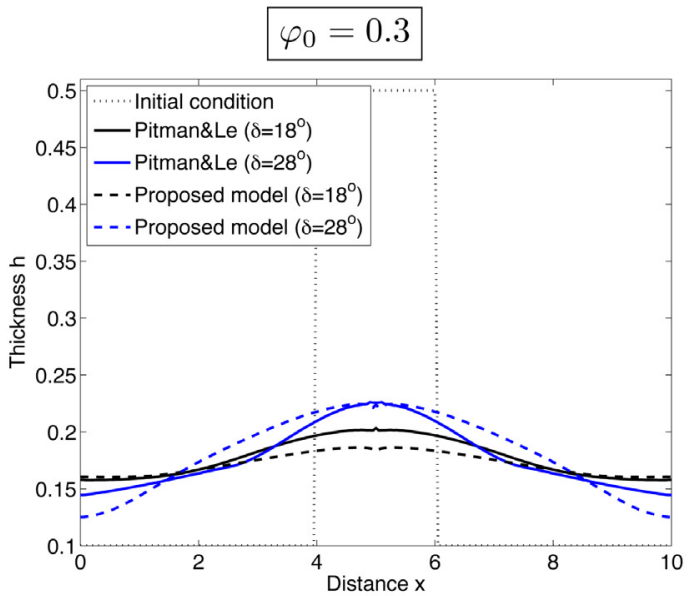

(a) $h(t=5 \mathrm{~s})$.

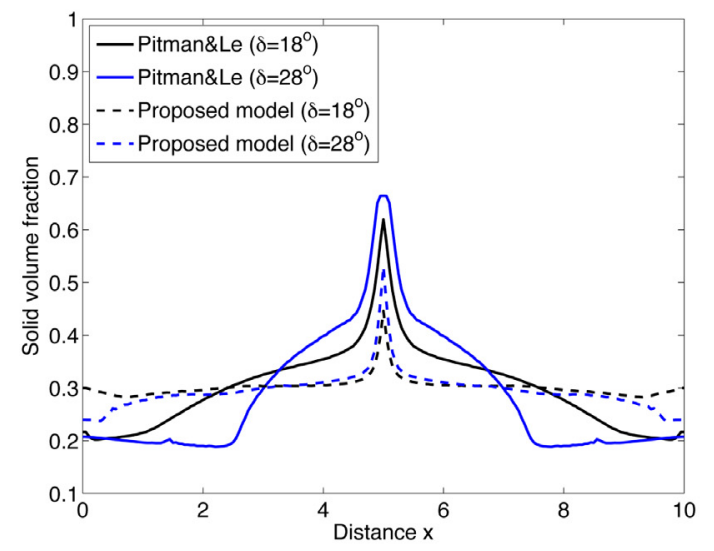

(c) $\varphi(t=5 \mathrm{~s})$.

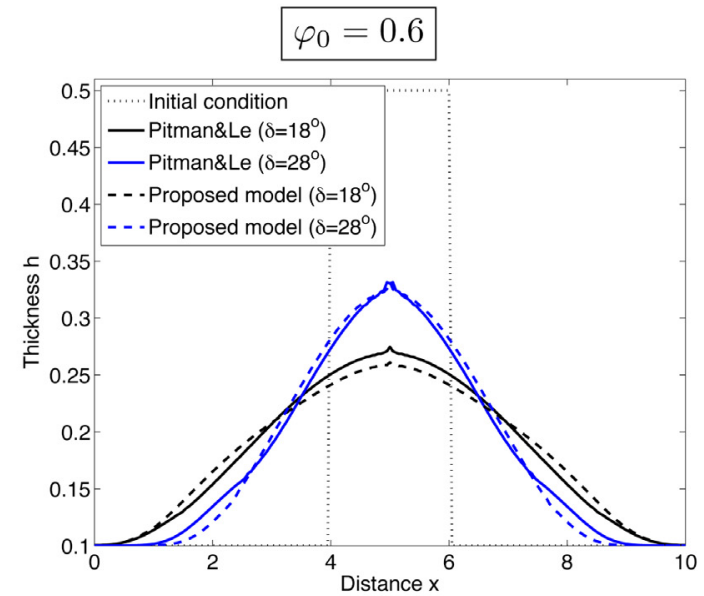

(b) $h(t=5 \mathrm{~s})$.

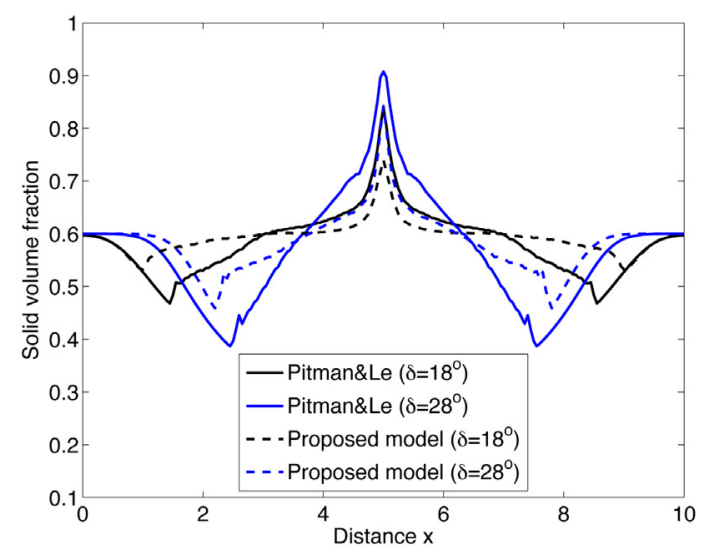

(d) $\varphi(t=5 \mathrm{~s})$.

Figure 6 . Test 1: comparison between the solutions obtained with the Pitman-Le and proposed models for the thickness of the mass $h(\mathrm{~m})$ and the volume fraction $\varphi$ as functions of the distance $x(\mathrm{~m})$ at time $t=5 \mathrm{~s}$ when the solid phase has already stopped, for the collapse of a rectangular granular mixture over a horizontal layer made of the same mixture. The initial volume fractions are: (left) $\varphi_{0}=0.3$; (right) $\varphi_{0}=0.6$. Here the friction law is the Richardson and Zaki drag force (RZ) with $m=1$.

two phases is greater (see Fig. 8). This difference is much larger in the PL model than in our model, leading to higher drag forces in the PL model. This explain why our model is less sensitive to the definition of the drag force (see Fig. 4).

\section{Evolution in time.}

Let us now look at the changes of the different quantities with time (Figs. 9-13 in which times $t=1,2,3$ and $5 \mathrm{~s}$ are represented with different colours). For these simulations, we use the Richardson and Zaki friction law with $m=1$ and the Coulomb friction angle $\delta=18^{\circ}$.

Note that even though the mass profiles change with time in a similar way for the two models (Fig. 9), there is strong difference between the two models for the changes of the volume fraction with time (Fig. 10), 


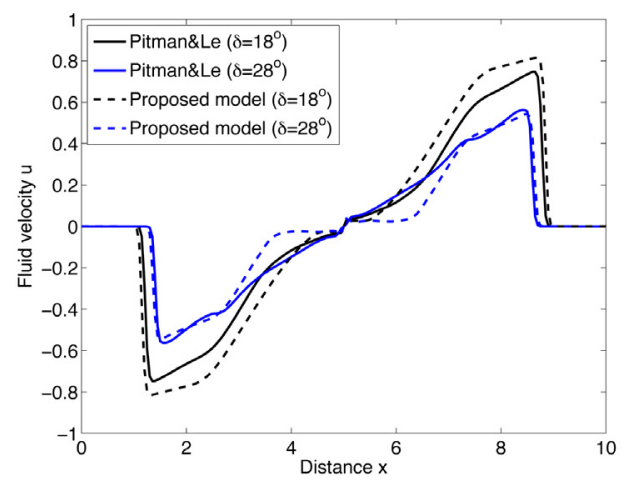

(a) $u(t=1.5 \mathrm{~s})$.

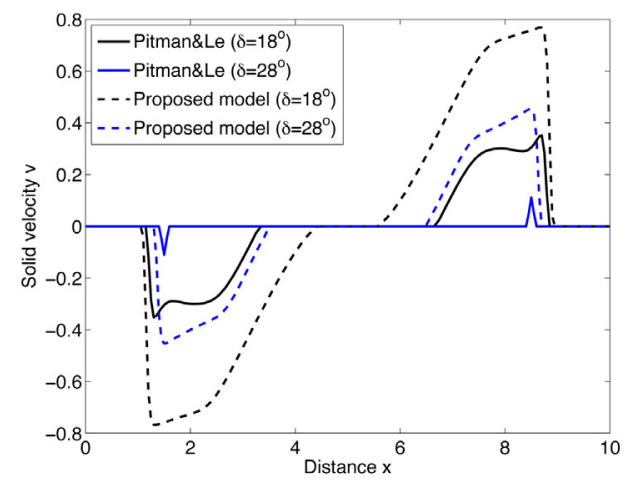

(c) $v(t=1.5 \mathrm{~s})$.

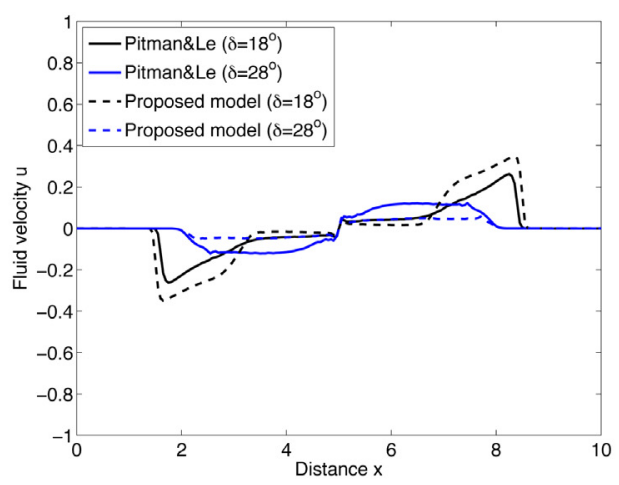

(b) $u(t=1.5 \mathrm{~s})$.

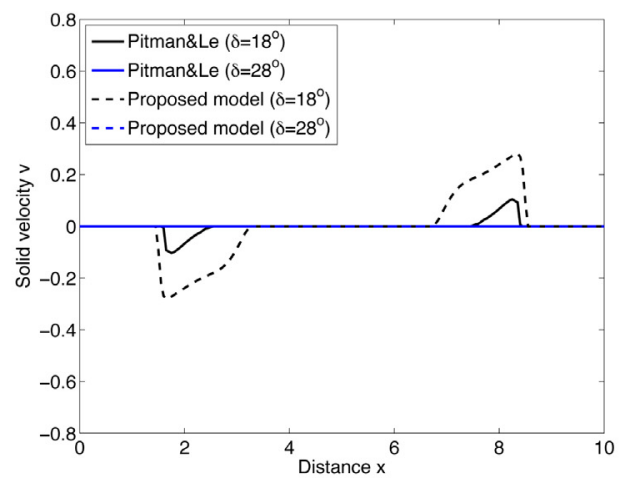

(d) $v(t=1.5 \mathrm{~s})$.

Figure 7. Test 1: comparison of the fluid and solid velocities $u\left(\mathrm{~m} \mathrm{~s}^{-1}\right)$ and $v\left(\mathrm{~m} \mathrm{~s}^{-1}\right)$ as functions of the distance $x(\mathrm{~m})$ for the Pitman-Le and proposed models at time $t=1.5 \mathrm{~s}$ for the collapse of a rectangular granular mixture over a horizontal layer made of the same mixture. The initial volume fractions are: (left) $\varphi_{0}=0.3$; (right) $\varphi_{0}=0.6$. Here the friction law is the Richardson and Zaki drag force (RZ) with $m=1$.

especially for $\varphi_{0}=0.3$. The volume fraction changes are much larger with the PL model. The fluid velocities are quite similar for the two models, whereas the solid velocity is higher with our model (Figs. 11 and 12). Finally Figure 13 shows the evolution of the new variable $\psi=\frac{p_{s_{\mid b+h}}}{\rho_{s}}$ (see Eq. (5.2)). As already mentioned, this variable is equal to zero in the PL model (where $p_{s_{\mid b+h}}=0$ is imposed as a boundary condition). For $\varphi_{0}=0.3$, the surface pressure of the solid phase increases until $t=1.4 \mathrm{~s}$ where it reaches its maximum value before decreasing. A similar behaviour is observed for $\varphi_{0}=0.6$. The final peak surface pressure is higher for $\varphi_{0}=0.6$. Note that the absolute value of the slope of $\psi$ is greater for $\varphi=0.6$ at all times. Moreover, the gradient of $\psi$ has the same sign as the gradient of $h$. This implies that the pressure gradient is larger for the solid phase and smaller for the fluid phase (see Eqs. (5.1c) and (5.1d)). Nevertheless, the gradient of $\psi$ is much smaller than the gradient of $g h$, corresponding to the gradient of the hydrostatic pressure.

\section{Residual energy term.}

In Section 4.1, we proved that the proposed model is compatible with a dissipative energy balance since the residual term $R_{e}$ in equation (4.10) is non-positive. We also argued in Remark 4.2 that this may not be true for 


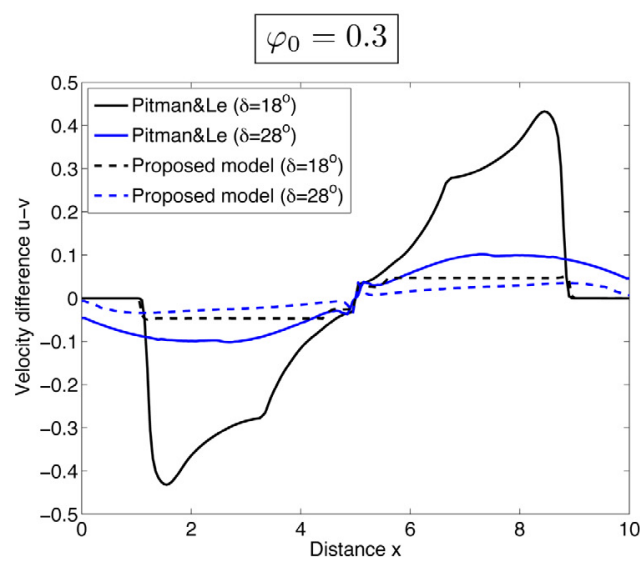

(a) $(u-v)(t=0.1 \mathrm{~s})$.

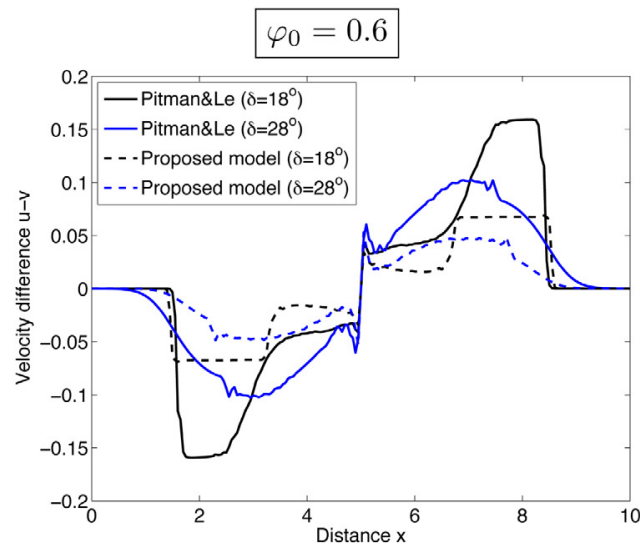

(b) $(u-v)(t=0.1 \mathrm{~s})$.

Figure 8 . Test 1: comparison of the difference of the fluid and solid velocities $(u-v)\left(\mathrm{m} \mathrm{s}^{-1}\right)$ for the Pitman-Le and proposed models at time $t=0.1 \mathrm{~s}$ for the collapse of a rectangular granular mixture over a horizontal layer made of the same mixture. The initial volume fractions are: (left) $\varphi_{0}=0.3$; (right) $\varphi_{0}=0.6$. Here the friction law is the Richardson and Zaki drag force (RZ) with $m=1$.

the PL model due to the additional term that has no fixed sign (the first term in the following equation):

$$
\begin{aligned}
R_{e}= & -\frac{1}{2} \varphi\left(\rho_{s}-\rho_{f}\right) g h \cos \theta \operatorname{div}(h(1-\varphi)(u-v)) \\
& -\tilde{\beta} h|u-v|^{2}-|v| \tan \delta \varphi\left(\rho_{s}-\rho_{f}\right) g h \cos \theta .
\end{aligned}
$$

Let us carry out a test on the numerical values of these terms. We chose the values $\varphi_{0}=0.3$ and $m=1$ for the Richardson and Zaki drag force. In Figure 14, we show the three terms involved in $R_{e}$ for times $t=0.5 \mathrm{k}$, $k=1 \mathrm{~s}, \ldots 10 \mathrm{~s}$, which we denote:

$$
\begin{aligned}
& R_{e 1}=-\frac{1}{2} \varphi\left(\rho_{s}-\rho_{f}\right) g h \cos \theta \operatorname{div}(h(1-\varphi)(u-v)) \\
& R_{e 2}=-\tilde{\beta} h|u-v|^{2} \\
& R_{e 3}=-|v| \tan \delta \varphi\left(\rho_{s}-\rho_{f}\right) g h \cos \theta .
\end{aligned}
$$

Note that the absolute value of $R_{e 2}$ is much larger than the values corresponding to $R_{e 1}$ and $R_{e 3}$. Moreover, the additional term $R_{e 1}$ has the same magnitude as the Coulomb friction term $R_{e 3}$, but with opposite sign. However, at the mass front, we observe that $\left|R_{e 1}\right|$ is greater than $\left|R_{e 3}\right|$. In Figure 15, we represent the total residual term $R_{e}=R_{e 1}+R_{e 2}+R_{e 3}$ for the same times. We observe that even if the friction effect between the two phases is large, the PL model provides positive values of the residual term in the mass front, i.e. it does not dissipate the energy.

\subsubsection{Test 2: Constant slope}

We consider here the collapse of a column made of a mixture of grains and fluid over a 20 metre long inclined bed of constant slope $\theta=10^{\circ}$. As in the previous case, we use the Richardson and Zaki drag force with $m=1$ and $v_{T}=0.143 \mathrm{~m} \mathrm{~s}^{-1}$. The Coulomb friction angle is $\delta=25^{\circ}$ and the initial conditions are defined as follows:

$$
h(t=0 \mathrm{~s})=\left\{\begin{array}{l}
0.5 \mathrm{~m} 4 \mathrm{~m} \leq x \leq 6 \mathrm{~m} \\
0.1 \mathrm{~m} \text { otherwise }
\end{array} ; \quad u(t=0 \mathrm{~s})=v(t=0 \mathrm{~s})=0 ; \quad \varphi(t=0 \mathrm{~s})=0.6 .\right.
$$




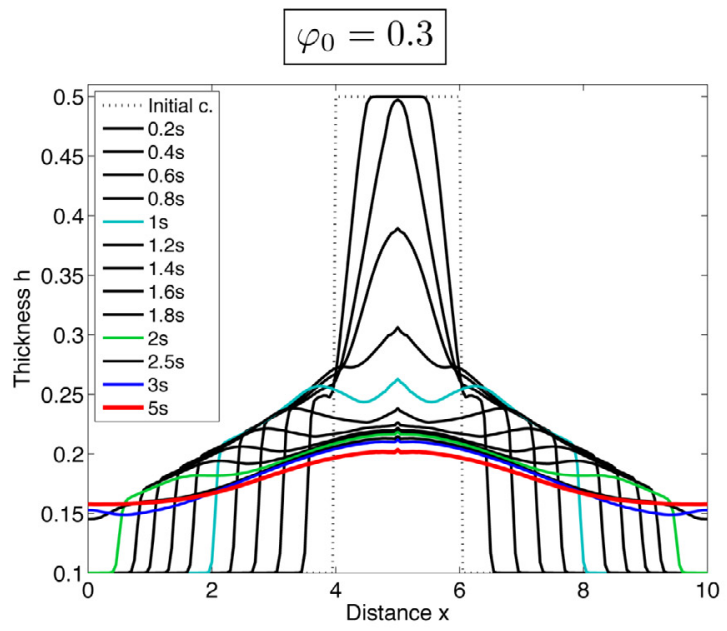

(a) h. Pitman-Le model.

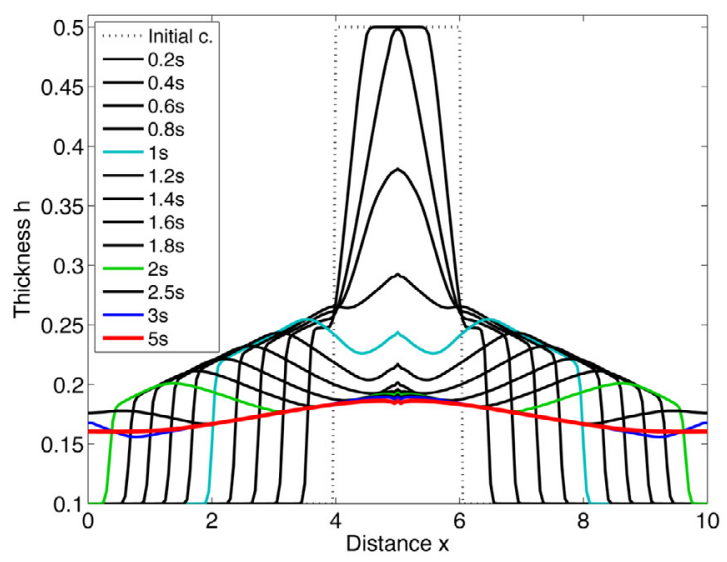

(c) h. Proposed model.

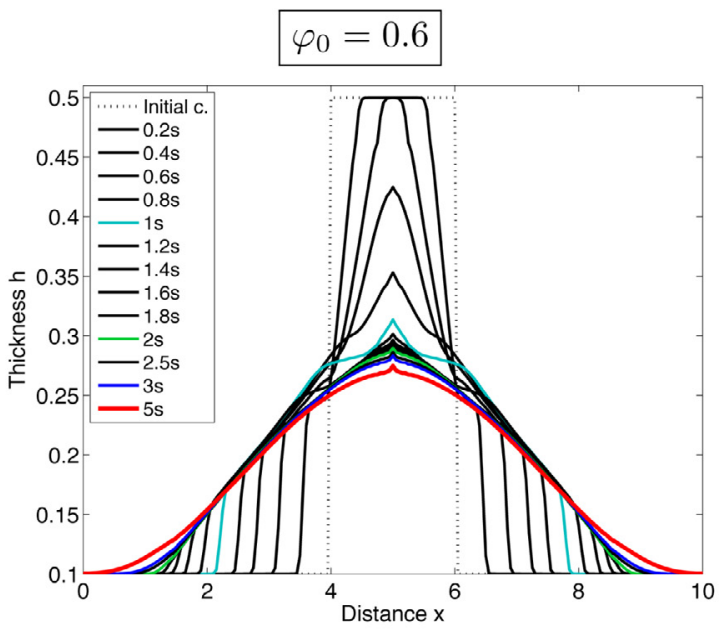

(b) $h$. Pitman-Le model.

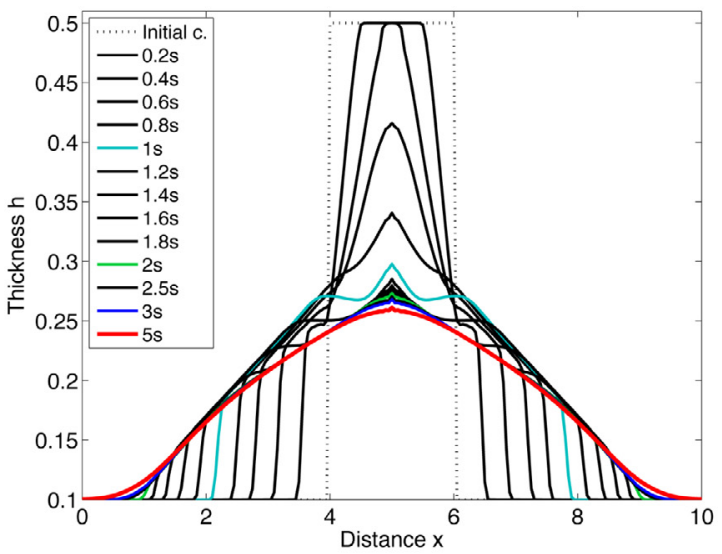

(d) $h$. Proposed model.

Figure 9. Test 1: the mass thickness $h(x, t)$ in meters as a function of the distance $x(\mathrm{~m})$ at different times for the Pitman-Le model (a-b) and for the model proposed here (c-d) for the collapse of a rectangular granular mixture over a horizontal layer made of the same mixture. The initial volume fractions are: (left) $\varphi_{0}=0.3$; (right) $\varphi_{0}=0.6$. Here the friction law is the Richardson and Zaki drag force (RZ) with $m=1$.

We consider open boundary conditions. That implies a constant supply of fluid and granular material at $x=0$. While we obtain a stationary solution with zero velocity for the solid phase, we observe that the velocity is not zero for the fluid phase (see Fig. 22). However, since we have a constant discharge for the fluid phase $(h(1-\varphi) u \approx 0.01)$, we have a stationary profile with a non-zero velocity for the fluid phase, corresponding to a small movement of the fluid between the pores of the grains produced by the continuous supply of the fluid at $x=0$ and by the slope of the bottom. In Figure 20 we make a comparison of the difference between the velocities of the two phases, $u-v$. In our model, this difference is smaller than in the PL model. As a result, the drag forces are smaller explaining the higher mobility of the spreading mass in our model.

Figures 16-19 compare the solutions obtained for the PL model and for the proposed model at different times. At time $(t=10 \mathrm{~s})$, the solid phase has already stopped. 


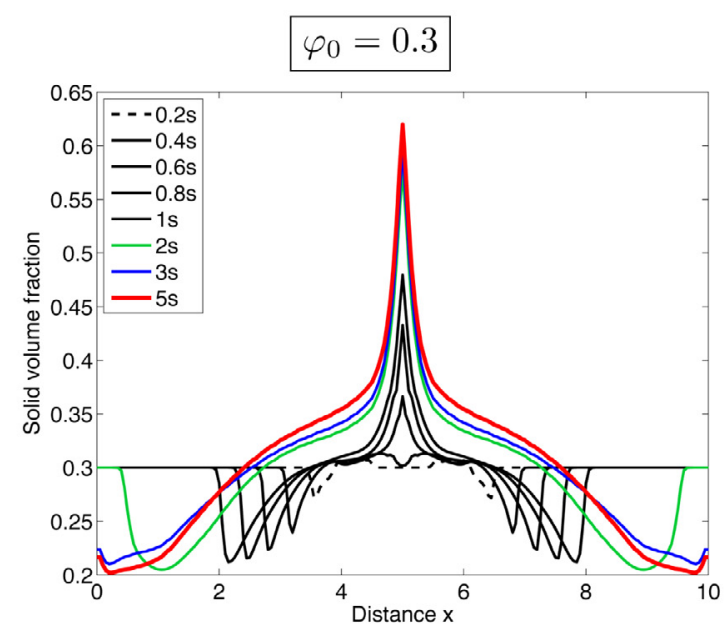

(a) $\varphi$. Pitman-Le model.

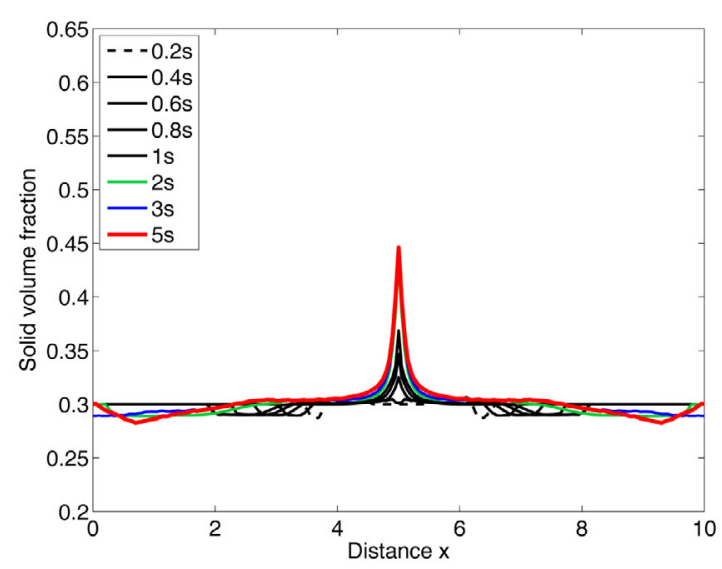

(c) $\varphi$. Proposed model.

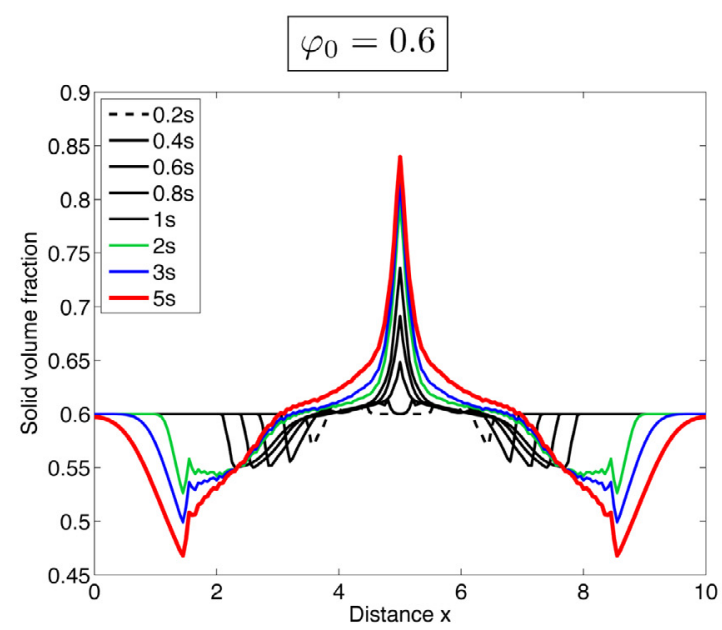

(b) $\varphi$. Pitman-Le model.

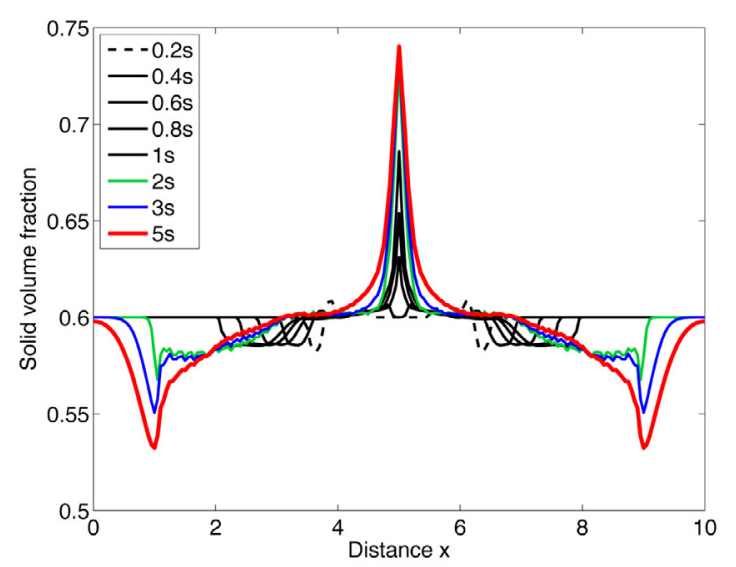

(d) $\varphi$. Proposed model.

Figure 10. Test 1: the solid volume fraction $\varphi(x, t)$ as a function of the distance $x(\mathrm{~m})$ at different times for the Pitman-Le model ( $a-b)$ and for the model proposed here (c-d) for the collapse of a rectangular granular mixture over a horizontal layer made of the same mixture. The initial volume fractions are: (left) $\varphi_{0}=0.3$; (right) $\varphi_{0}=0.6$. Here the friction law is the Richardson and Zaki drag force (RZ) with $m=1$.

At the very beginning of the collapse, the two models give very similar results. This could be related to the predominance of pressure gradient terms and inertial terms compared to the friction terms at the onset of the collapse as observed for example in [24]. However, at later times (e.g.t $=3 \mathrm{~s}$ ), the two models show differences. In particular our model predicts a steeper front that the PL model. The spreading mass and the final mass profiles $(t=10 \mathrm{~s})$ are more extended. The runout distance is about $12 \%$ larger with our model and the deposit is more uniform (Fig. 16). For the solid volume fraction, we observe the same behaviour as in Test 1: (i) the two models behave qualitatively in the same way, (ii) even when the thickness profiles are very similar, as at time $t=1 \mathrm{~s}$, the solid volume fraction $\varphi$ is quite different for the two models (see Fig. 17a), (iii) the volume fraction varies less in our model and the peak values of the volume fraction are smaller. The global trend is that the tail and the front of the mass are more fluidized (dropping to $\varphi=0.4$ ) than the central part of the mass that has a 


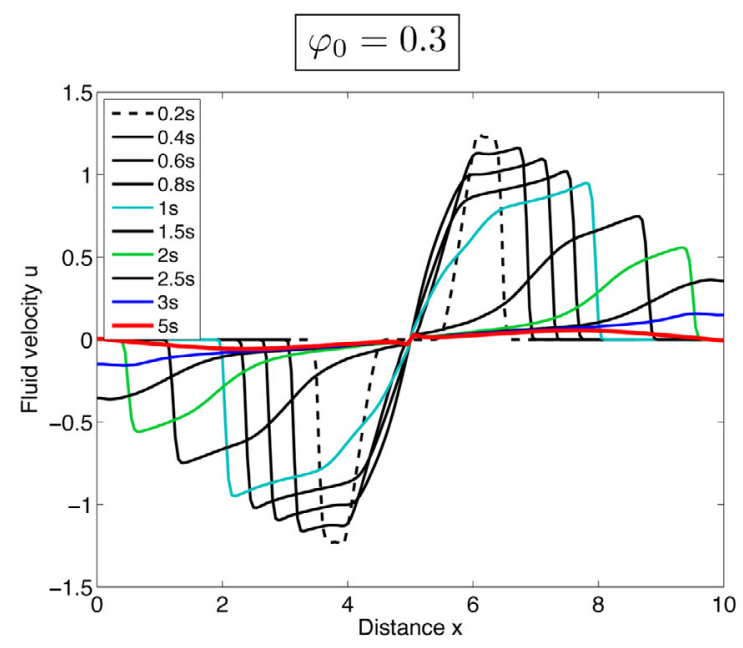

(a) $u$. Pitman-Le model.

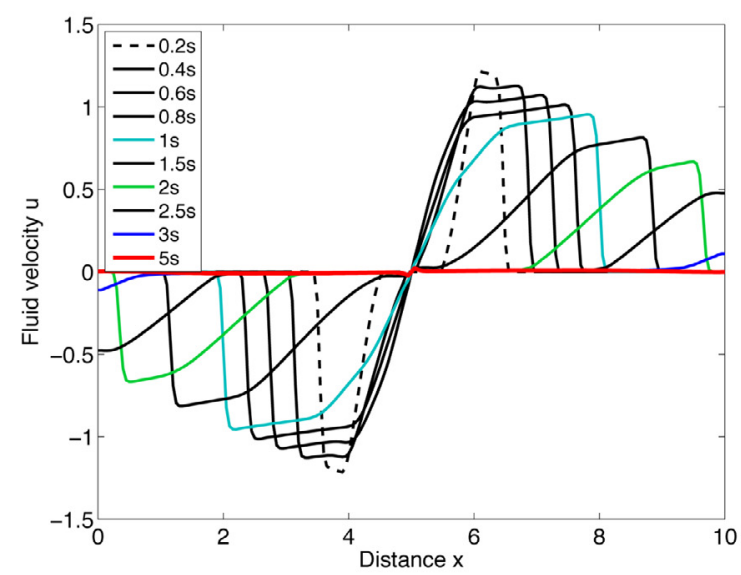

(c) $u$. Proposed model.

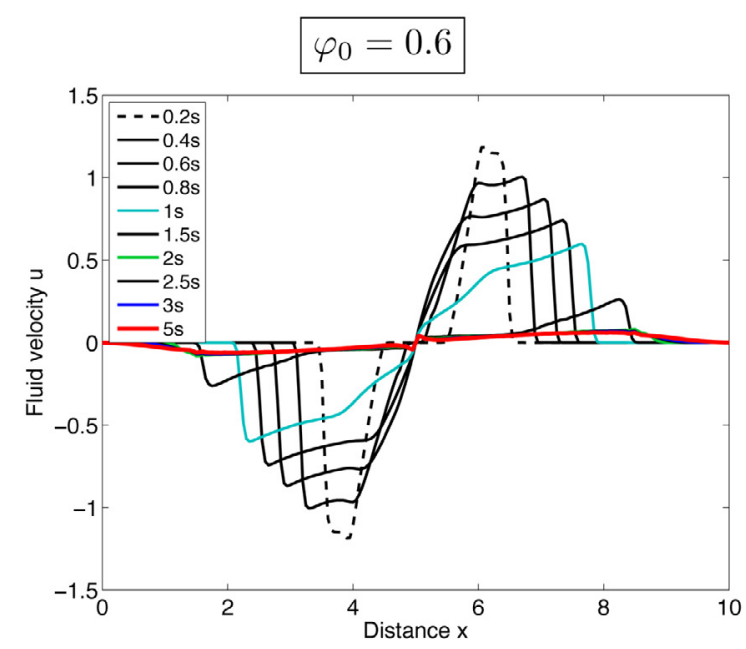

(b) u. Pitman-Le model.

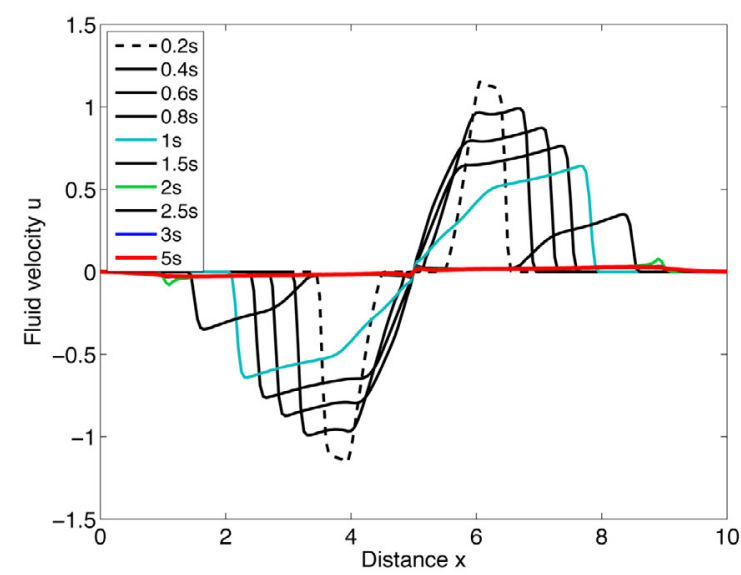

(d) $u$. Proposed model.

Figure 11. Test 1: the fluid velocity $u(x, t)$ in $\mathrm{m} \mathrm{s}^{-1}$ as a function of the distance $x(\mathrm{~m})$ at different times for the Pitman-Le model (a-b) and for the model proposed here (c-d) for the collapse of a rectangular granular mixture over a horizontal layer made of the same mixture. The initial volume fractions are: (left) $\varphi_{0}=0.3$; (right) $\varphi_{0}=0.6$. Here the friction law is the Richardson and Zaki drag force (RZ) with $m=1$.

volume fraction reaching up to $\varphi \approx 0.8$. Figures 18 and 19 show that the velocities of both phases are generally higher for the proposed model (more than $20 \%$ is some cases). While the solid phase is completely stopped at time $t=7 \mathrm{~s}$ for the PL model, the solid phase continues to move until $t=8 \mathrm{~s}$ for the proposed model.

Finally, let us look at the change of all the solutions of the problem with time (Figs. 21-23). The main features are the smaller variation of the volume fraction with our model, the higher velocities of the solid phase and the fact that the solid phase stops later than with the PL model. Note that with the PL model, the blue line corresponding to time $t=7 \mathrm{~s}$ is very close to the final time $t=10 \mathrm{~s}$ for the fluid phase and identical for the solid phase solutions, while they remain different for the proposed model (Fig. 22). The sign of the surface pressure of the solid phase changes along the mass profile, going from positive values at the tail to negative 


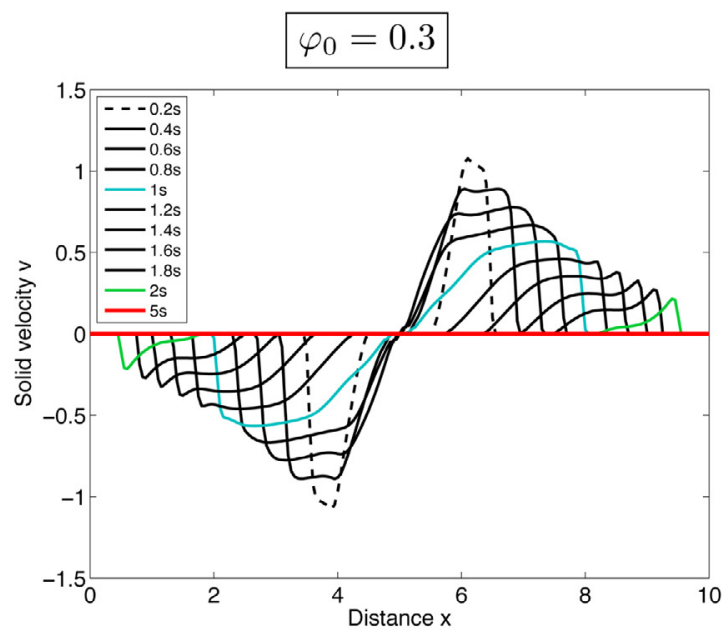

(a) v. Pitman-Le model.

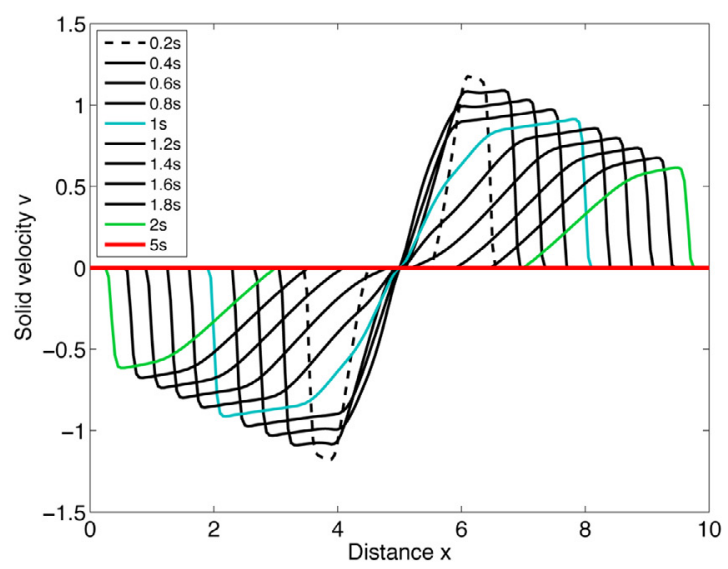

(c) v. Proposed model.

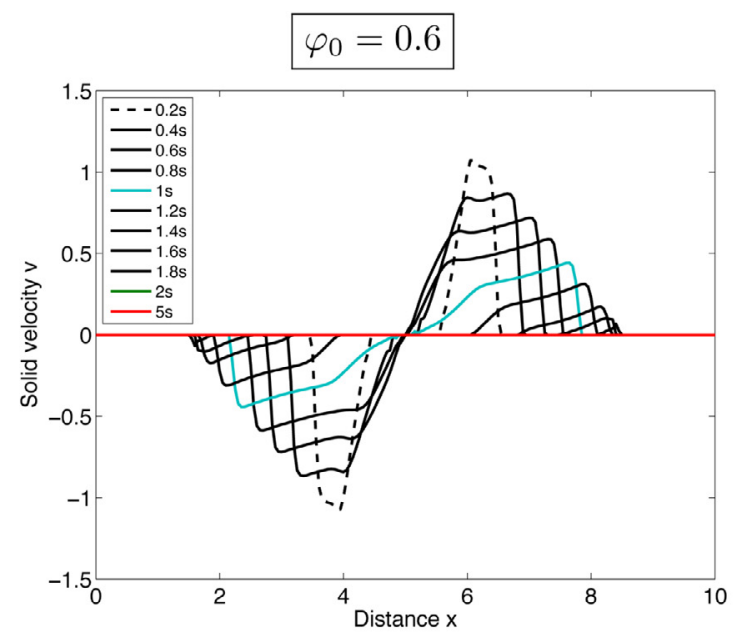

(b) v. Pitman-Le model.

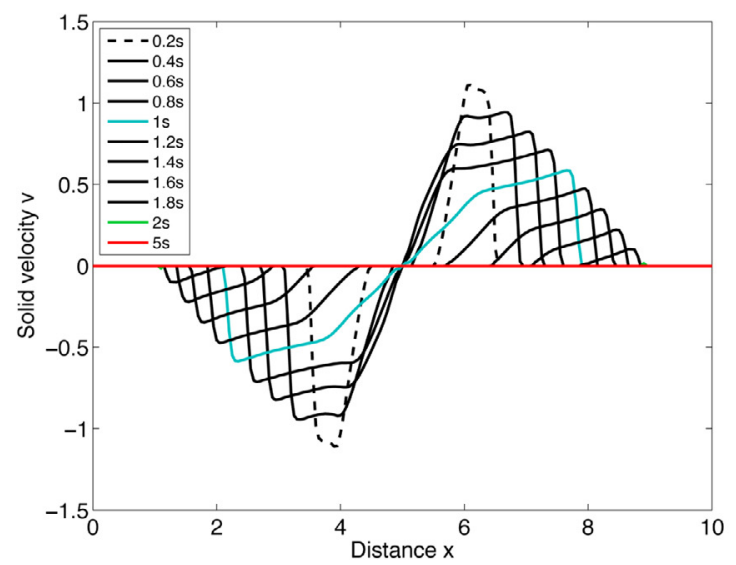

(d) v. Proposed model.

Figure 12. Test 1: the solid velocity $v(x, t)$ in $\mathrm{m} \mathrm{s}^{-1}$ as a function of the distance $x(\mathrm{~m})$ at different times for the Pitman-Le model (a-b) and for the model proposed here (c-d) for the collapse of a rectangular granular mixture over a horizontal layer made of the same mixture. The initial volume fractions are: (left) $\varphi_{0}=0.3$; (right) $\varphi_{0}=0.6$. Here the friction law is the Richardson and Zaki drag force (RZ) with $m=1$.

values behind the front (Fig. 23a). This would suggest compression of the solid phase near the tail and dilation behind the front. Finally, note that the magnitude of $\psi$ is small compared to the hydrostatic pressure gradient. In Figure 23b, we compare the value of $\psi$ with $g(x \tan \theta+h)$ for $t=10 \mathrm{~s}$.

\section{Conclusion}

In this paper, we propose a simplified thin layer (i.e. shallow) model to study hydrostatic two-phase avalanche problems that is compatible with a dissipative energy balance. It is deduced from Jackson's model. Analysis of this 3D model reveals that it contains more unknowns than equations. Therefore a closure equation must be 


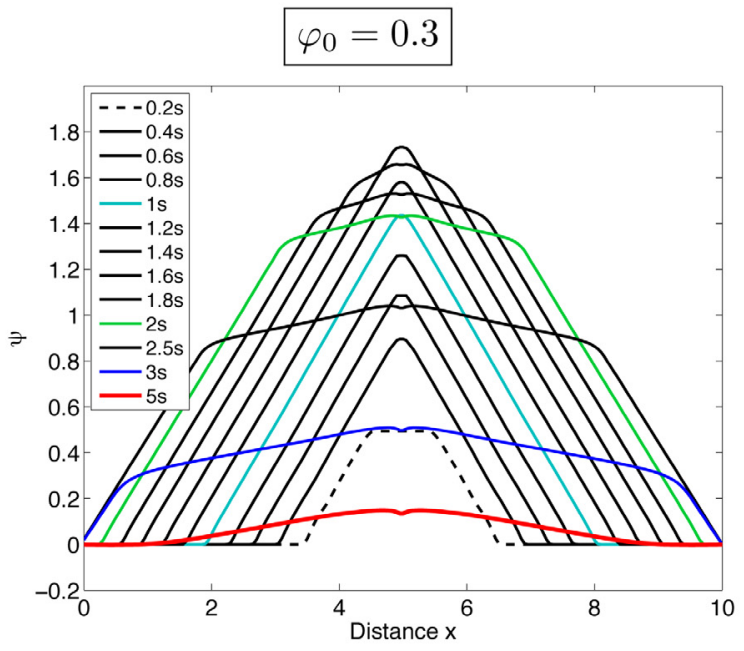

(a) $\psi(x, t)$.

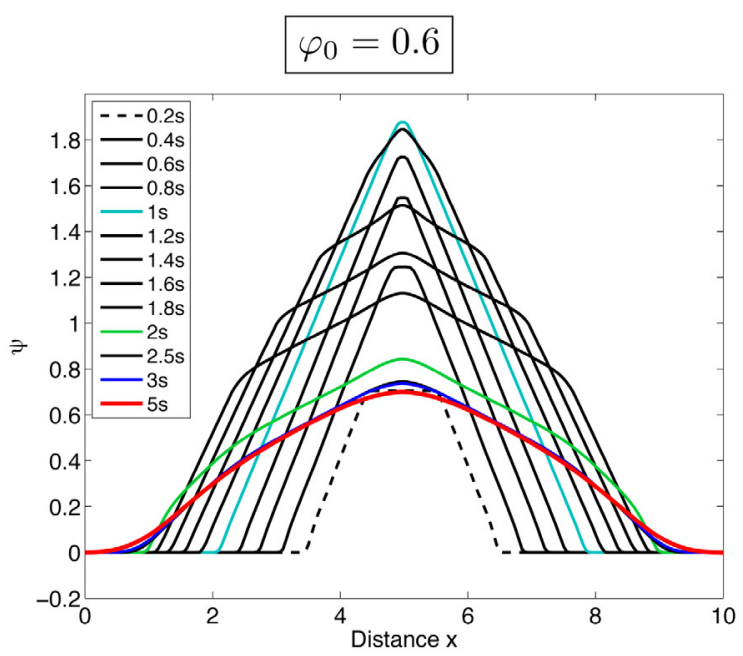

(b) $\psi(x, t)$.

Figure 13. Test 1: the variable $\psi(x, t)$ in $\mathrm{m}^{2} \mathrm{~s}^{-2}$ in the proposed model at different times for the collapse of a rectangular granular mixture over a horizontal layer made of the same mixture. The initial volume fractions are: (left) $\varphi_{0}=0.3 ;$ (right) $\varphi_{0}=0.6$. Here the friction law is the Richardson and Zaki drag force (RZ) with $m=1$.

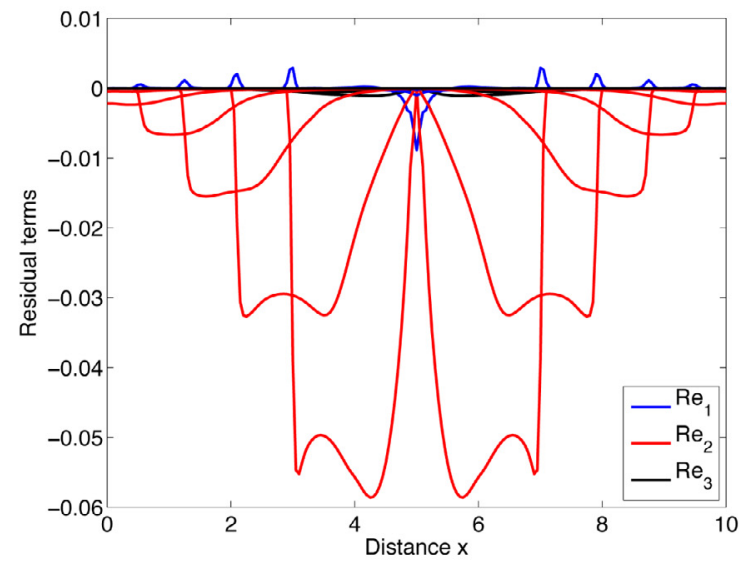

(a) Residual terms.

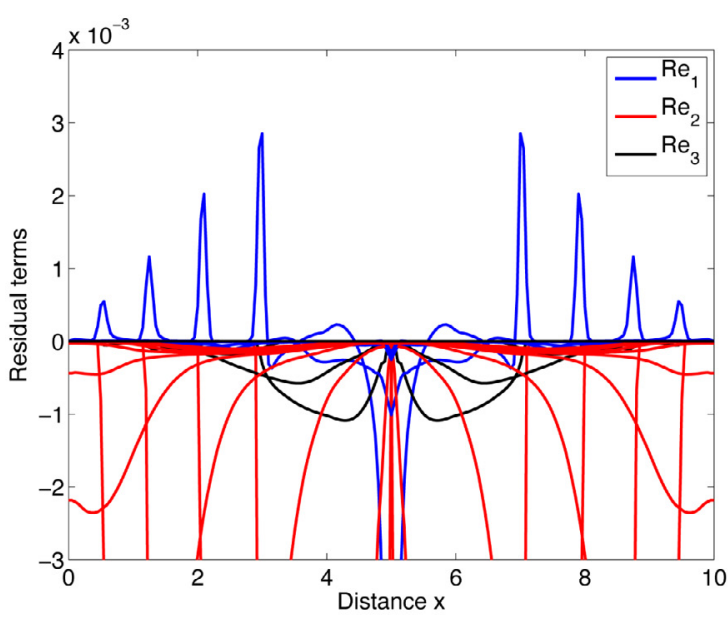

(b) Residual terms (zoom).

Figure 14. Test 1: values of the terms involved in the residual energy (see Eq. (5.10)) as a function of the distance $x(\mathrm{~m})$, for the collapse of a rectangular granular mixture over a horizontal layer made of the same mixture at the different times $t=0.5 \mathrm{k}$ with $k=1 \mathrm{~s}, \ldots 10 \mathrm{~s}$. The initial volume fraction is $\varphi_{0}=0.3$ and the friction law is the Richardson and Zaki drag force (RZ) with $m=1$. 


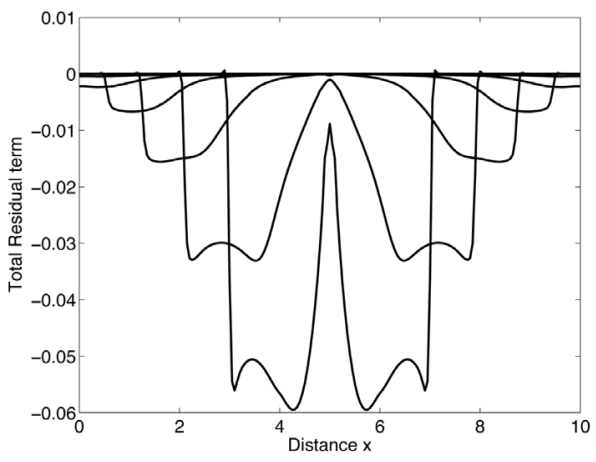

(a) Total residual term $R_{e}$.

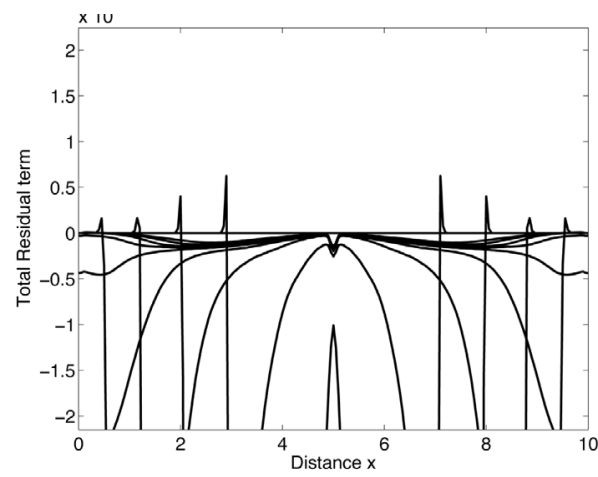

(b) Total residual term $R_{e}$ (zoom).

Figure 15. Test 1: values of the total residual term as a function of the distance $x(\mathrm{~m})$ for the collapse of a rectangular granular mixture over a horizontal layer made of the same mixture at the different times $t=0.5 \mathrm{k}$ with $k=1 \mathrm{~s}, \ldots 10 \mathrm{~s}$. The initial volume fraction is $\varphi_{0}=0.3$ and the friction law is the Richardson and Zaki drag force (RZ) with $m=1$.

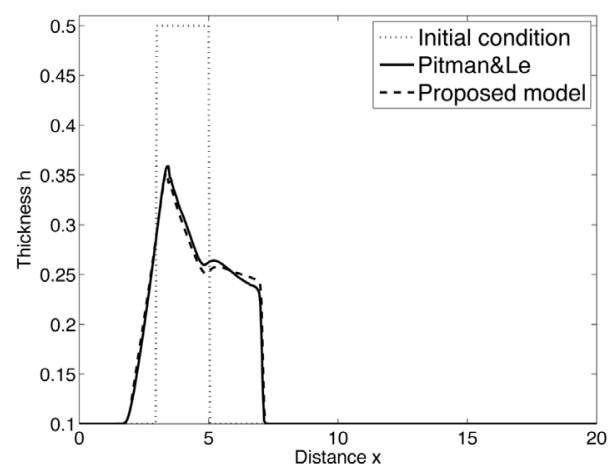

(a) $h(t=1 \mathrm{~s})$.

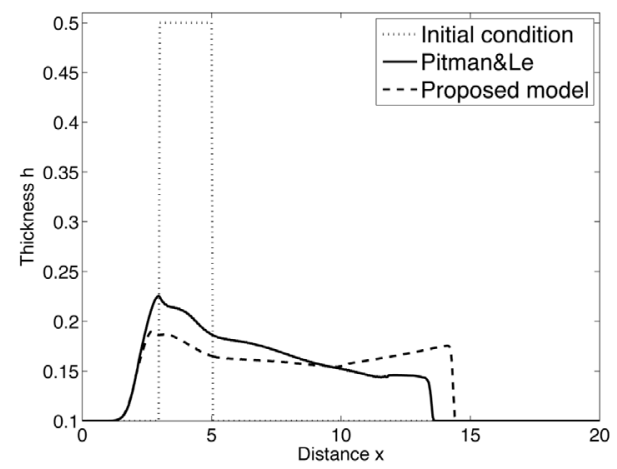

(c) $h(t=5 \mathrm{~s})$.

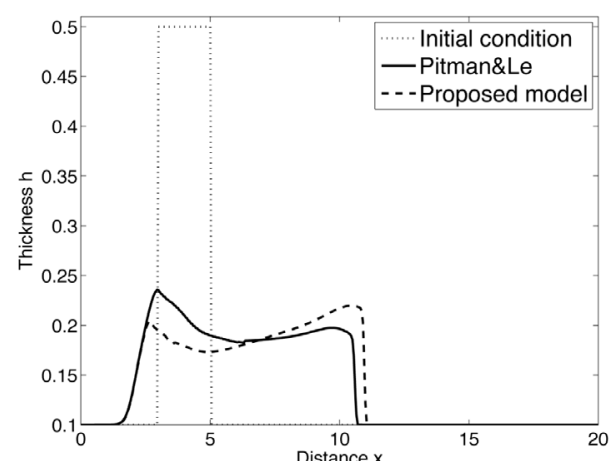

(b) $h(t=3 \mathrm{~s})$.

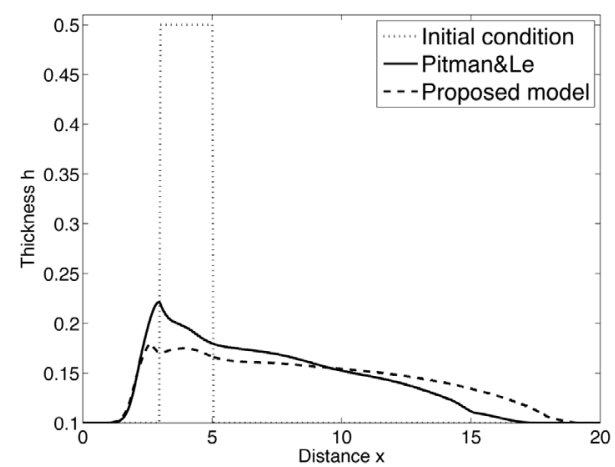

(d) $h(t=10 \mathrm{~s})$.

Figure 16. Test 2: comparison of the thickness profiles of the mass $h(x, t)$ in meters as a function of the distance $x(\mathrm{~m})$ for the Pitman-Le and proposed models, at time $t=1,3,5,10 \mathrm{~s}$ (at $t=10 \mathrm{~s}$ the solid phase has already stopped), for the collapse of a rectangular granular mixture over an inclined layer $\left(\theta=10^{\circ}\right)$ made of the same mixture. The initial volume fraction is $\varphi_{0}=0.6$. Here the friction law is the Richardson and Zaki drag force (RZ) with $m=1$. 


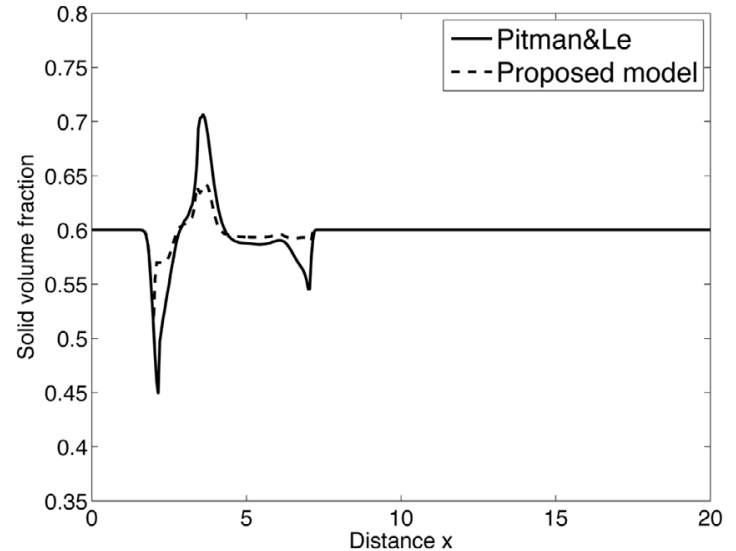

(a) $\varphi(t=1 \mathrm{~s})$.

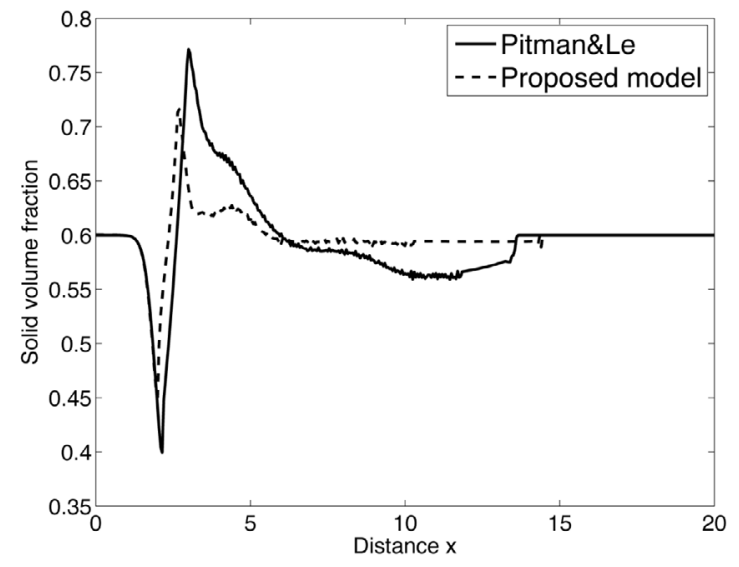

(c) $\varphi(t=5 \mathrm{~s})$.

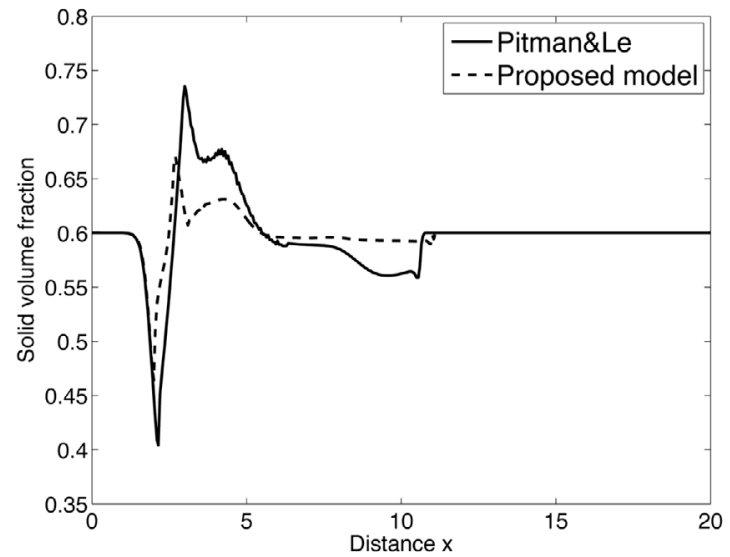

(b) $\varphi(t=3 \mathrm{~s})$.

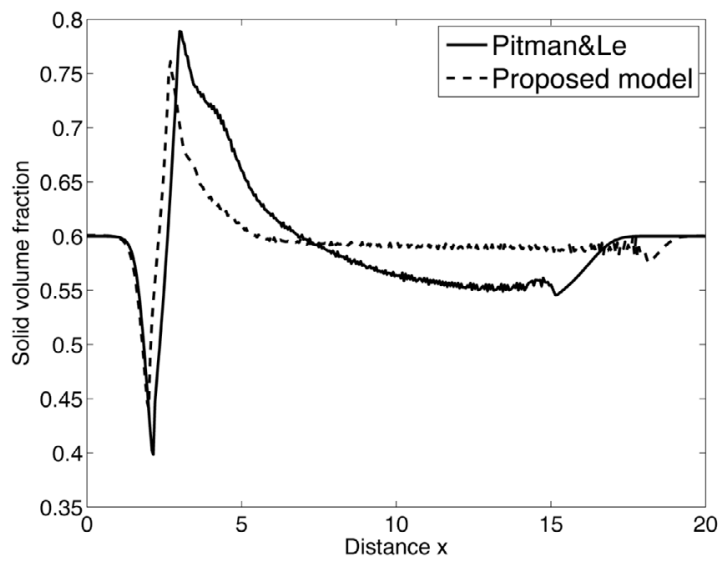

(d) $\varphi(t=10 \mathrm{~s})$.

FiguRE 17. Test 2: comparison of the solid volume fraction of the mixture $\varphi(x, t)$ as a function of the distance $x(\mathrm{~m})$ for the Pitman-Le and proposed models, at time $t=1,3,5,10 \mathrm{~s}$ (at $t=10 \mathrm{~s}$ the solid phase has already stopped), for the collapse of a rectangular granular mixture over an inclined layer $\left(\theta=10^{\circ}\right)$ made of the same mixture. The initial volume fraction is $\varphi_{0}=0.6$. Here the friction law is the Richardson and Zaki drag force (RZ) with $m=1$.

added to Jackson's model to obtain a well-posed system. This may not be apparent with a depth-averaged model with hydrostatic pressure, such as the one proposed by Pitman and Le [38]. Indeed, if we assume hydrostatic pressure for both phases, they are related by their boundary condition. In this case, imposing zero atmospheric pressure for both phases can be seen as the corresponding closure equation. Nevertheless, the model that is deduced does not have a dissipative energy balance. The main difference between the model that we propose in this paper and the Pitman-Le model comes from the boundary condition on the free surface. In the proposed model, we only impose that the sum of the pressures of the two phases is zero and not each of them. This introduces new unknown in the simplified model. As a closure equation for the 3D system, we consider incompressibility of the solid phase. This closure relation is consistent with the hydrostatic pressure assumption. The numerical tests presented here show that, overall, the changes of the profiles of the flowing mass with time are similar for the Pitman-Le model and the model proposed here. The qualitative behaviour of the solid volume fraction and 


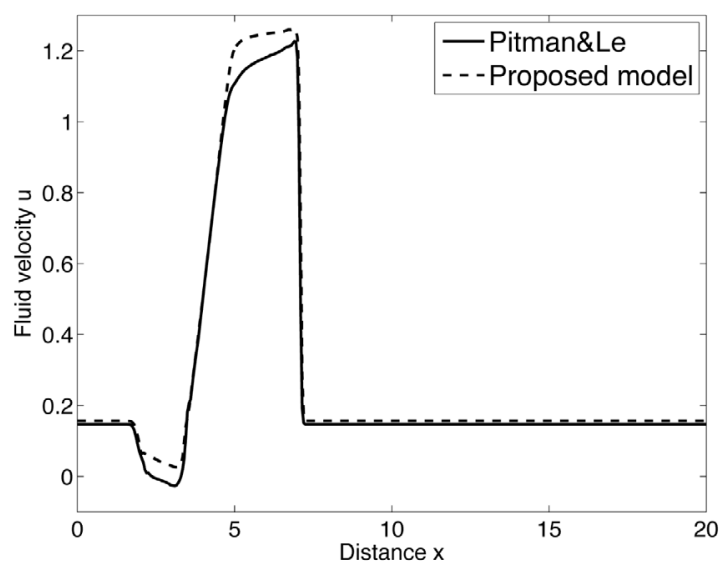

(a) $u(t=1 \mathrm{~s})$.

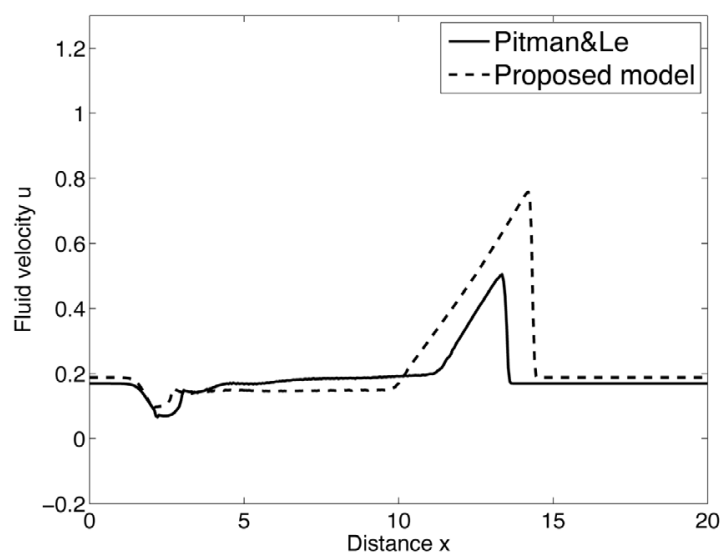

(c) $u(t=5 \mathrm{~s})$.

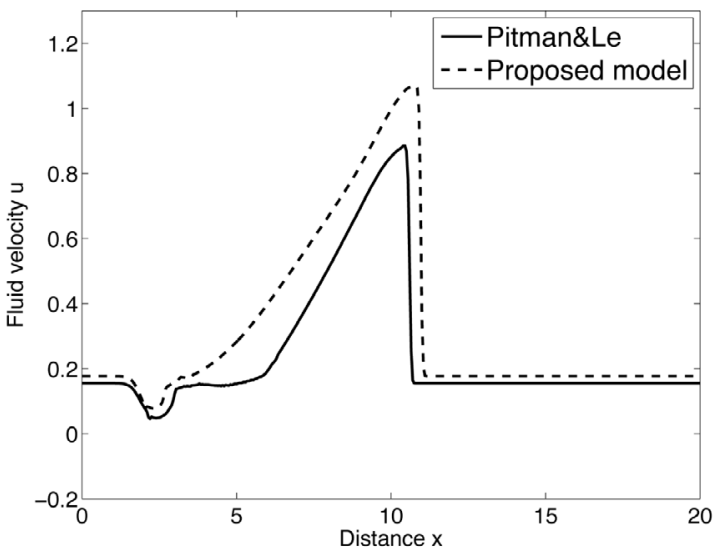

(b) $u(t=3 \mathrm{~s})$.

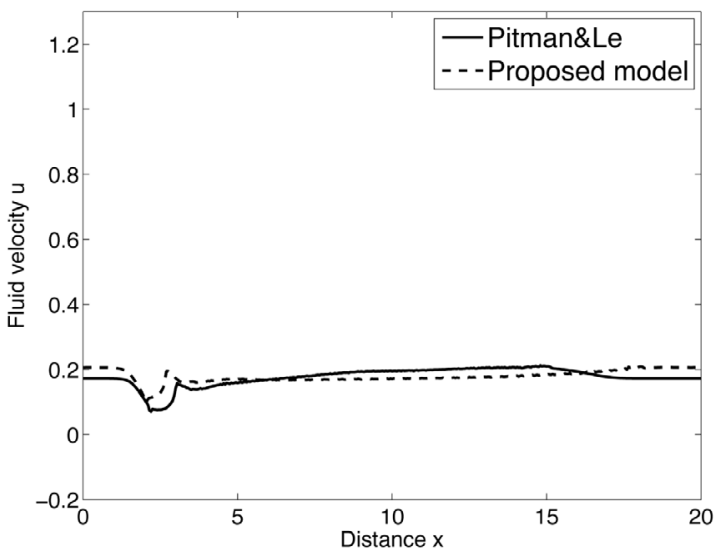

(d) $u(t=10 \mathrm{~s})$.

Figure 18. Test 2: comparison of the velocity of the fluid phase $u(x, t)$ in $\mathrm{m} \mathrm{s}^{-1}$ as a function of the distance $x(\mathrm{~m})$ for the Pitman-Le and proposed models, at time $t=1,3,5,10 \mathrm{~s}$ (at $t=10 \mathrm{~s}$ the solid phase has already stopped), for the collapse of a rectangular granular mixture over an inclined layer $\left(\theta=10^{\circ}\right)$ made of the same mixture. The initial volume fraction is $\varphi_{0}=0.6$. Here the friction law is the Richardson and Zaki drag force (RZ) with $m=1$.

the solid and fluid velocities is the same for both models. However, with the model presented here, the solid volume fraction varies less, the solid phase velocity is generally higher and the difference between the velocities of the two phases is smaller, leading to smaller drag forces between the two phases. This induces significant differences in the profile of the spreading mass and of the deposit with runout distances more than $10 \%$ larger and velocities that could be more than $20 \%$ heigher in the simple test of granular collapse over inclined plane performed here. While it is quite difficult to measure experimentally or in the field the fluid and solid velocities, the use of seismic waves generated by debris flows or avalanches may be a new way to discriminate between these two models $([9,31])$.

An advantage of our model is that the closure equation (i.e. incompressibility of the solid phase) is explicitly imposed, making it possible to derive physical interpretation of our results while in the PL model, the behavior is dictated by the imposed zero-pressure at the surface of each phase, without any description of the mechanical 


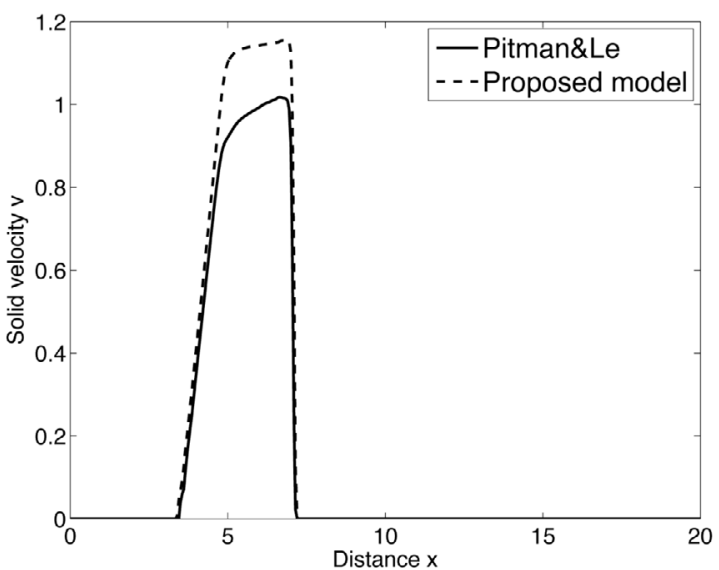

(a) $v(t=1 \mathrm{~s})$.

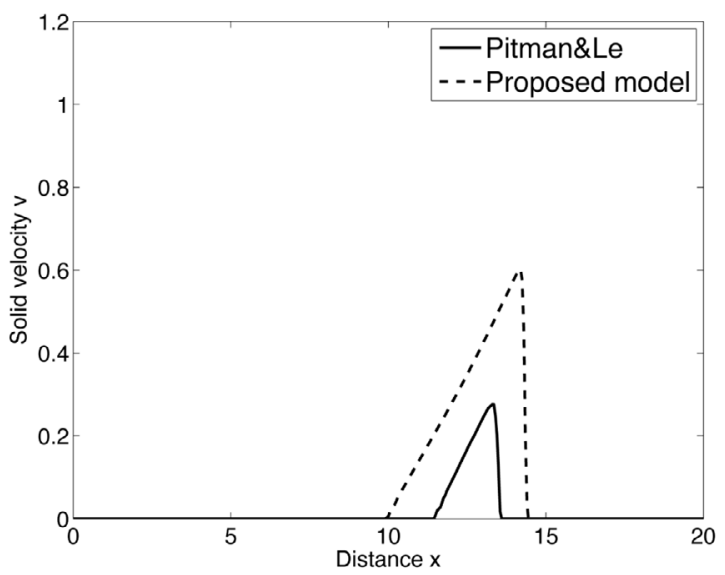

(c) $v(t=5 \mathrm{~s})$.

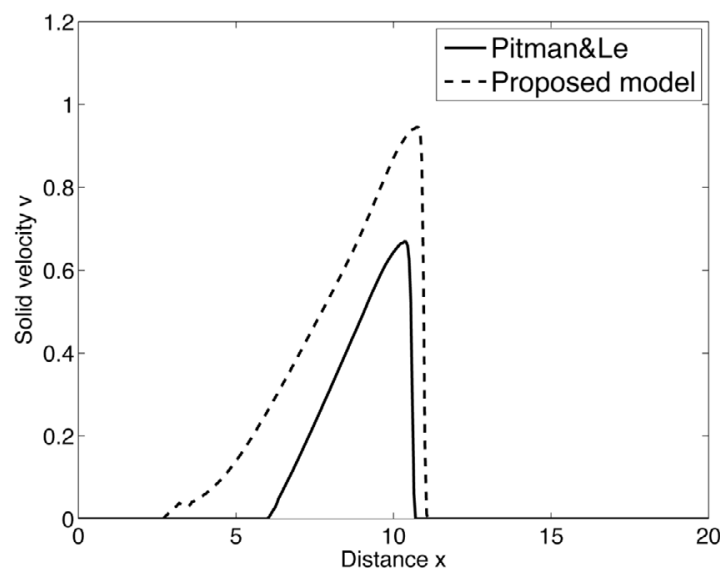

(b) $v(t=3 \mathrm{~s})$.

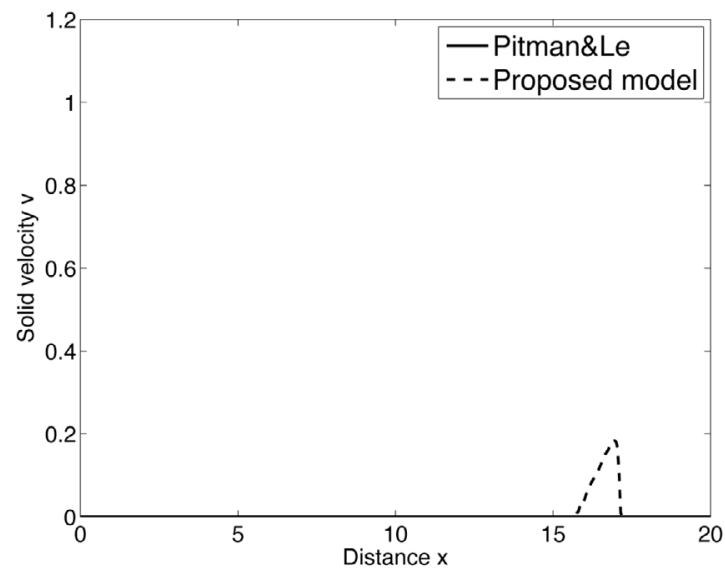

(d) $v(t=7 \mathrm{~s})$.

Figure 19. Test 2: comparison of the velocity of the solid phase $v(x, t)$ in $\mathrm{m} \mathrm{s}^{-1}$ as a function of the distance $x(\mathrm{~m})$ for the Pitman-Le and proposed models, at time $t=1,3,5,7 \mathrm{~s}($ at $t=10 \mathrm{~s}$ the solid phase has already stopped), for the collapse of a rectangular granular mixture over an inclined layer $\left(\theta=10^{\circ}\right)$ made of the same mixture. The initial volume fraction is $\varphi_{0}=0.6$. Here the friction law is the Richardson and Zaki drag force (RZ) with $m=1$.

properties of the solid phase. It is interesting to see the significant differences between the two models even though the surface pressure of each phase is very small in our model.

This analysis is largely driven by the kinematic boundary conditions that imposes the two phases to fill the same domain. However in debris flows, the fluid phase surface can be higher or lower than the solid phase surface, due to the relative motion between these two phases. This is expected to be significant in particular when compression/dilation of the solid phase occur. Because the models seem to be very sensitive to what happens at the surface, even small variations of the fluid and solid surfaces could have a strong impact on the results. Further analysis of the equations should be performed with relaxation of these boundary conditions and including a more realistic closure relation related to the compression/dilation of the granular phase. 


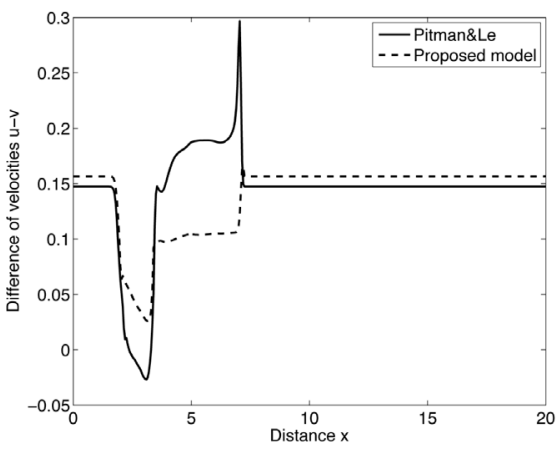

(a) $u-v(t=1 \mathrm{~s})$.

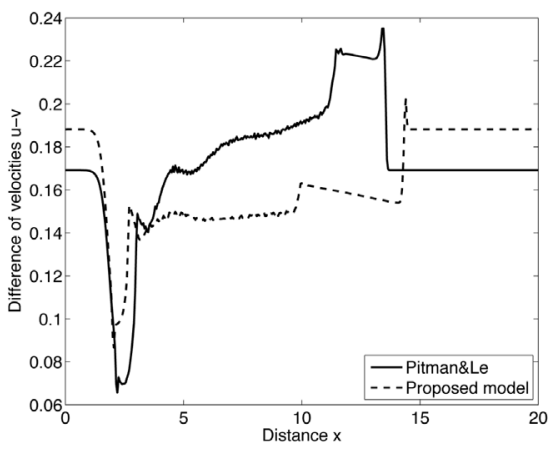

(b) $u-v(t=5 \mathrm{~s})$.

Figure 20. Test 2: comparison of the velocity difference of the fluid $u(x, t)$ and solid phase $v(x, t)$ in $\mathrm{m} \mathrm{s}^{-1}$ as functions of the distance $x(\mathrm{~m})$ for the Pitman-Le and proposed models, at time $t=1$ and $5 \mathrm{~s}$, for the collapse of a rectangular granular mixture over an inclined layer $\left(\theta=10^{\circ}\right)$ made of the same mixture. The initial volume fraction is $\varphi_{0}=0.6$. Here the friction law is the Richardson and Zaki drag force (RZ) with $m=1$.

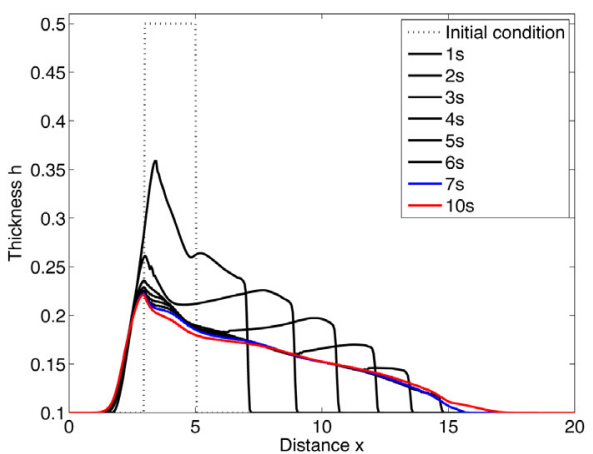

(a) $h$. Pitman-Le model.

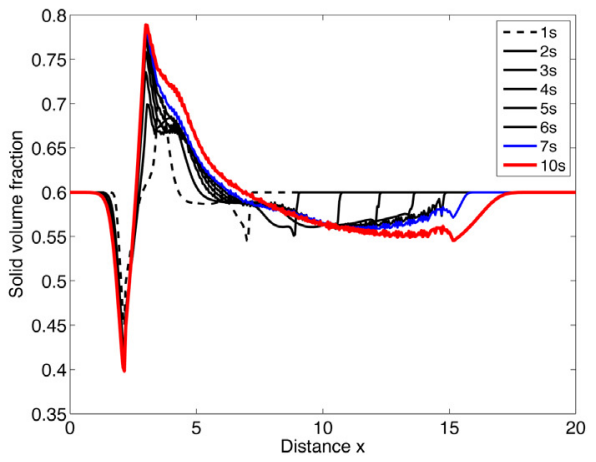

(c) $\varphi$. Pitman-Le model.

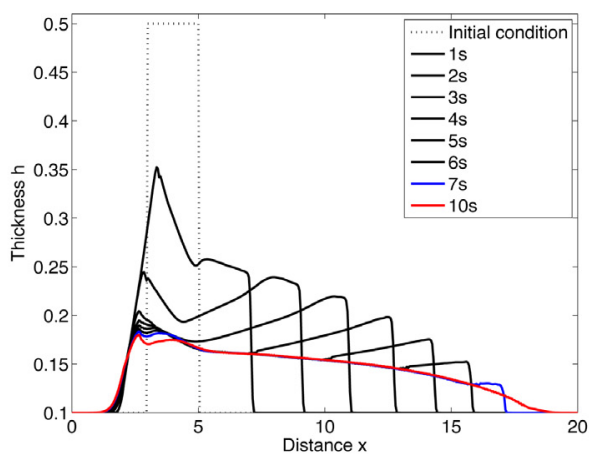

(b) h. Proposed model.

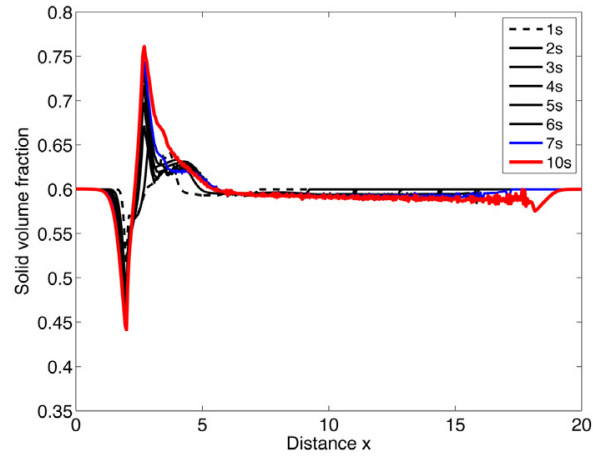

(d) $\varphi$. Proposed model.

Figure 21. Test 2: the mass thickness $h(x, t)$ in meters as a function of the distance $x(\mathrm{~m})(\mathrm{a}-\mathrm{b})$ and of the solid volume fraction $\varphi(x, t)$ as a function of the distance $x(\mathrm{~m})(\mathrm{c}-\mathrm{d})$ at different times for the collapse of a rectangular granular mixture over an inclined layer $\left(\theta=10^{\circ}\right)$ made of the same mixture. The initial volume fraction is $\varphi_{0}=0.6$. Here the friction law is the Richardson and Zaki drag force (RZ) with $m=1$. 


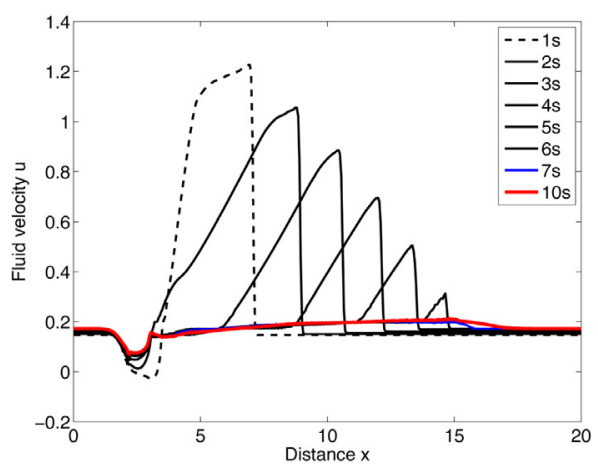

(a) $u$. Pitman-Le model.

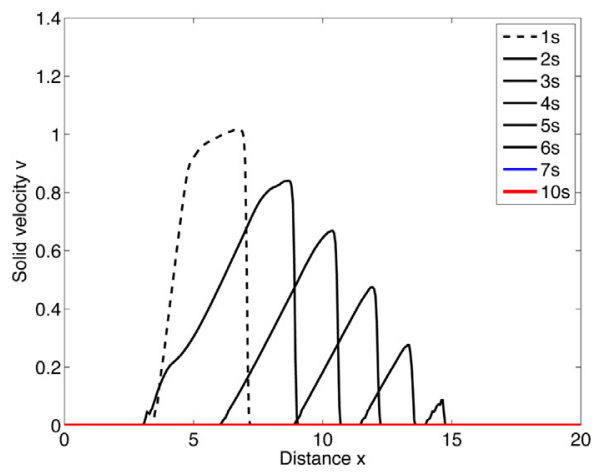

(c) v. Pitman-Le model.

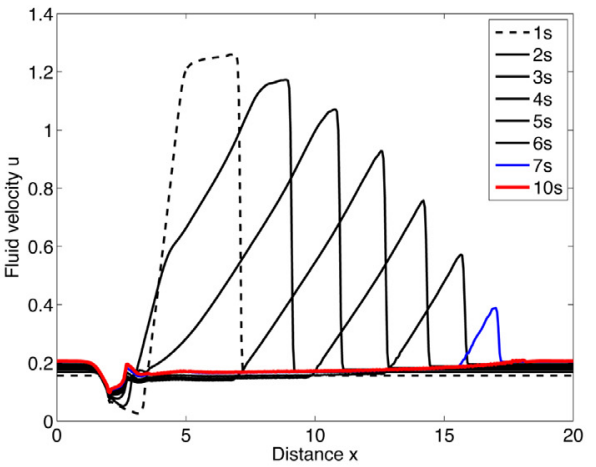

(b) $u$. Proposed model.

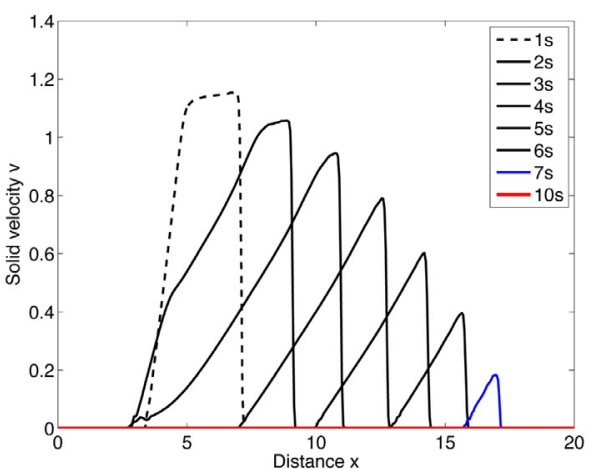

(d) v. Proposed model.

Figure 22. Test 2: the velocity of the fluid phase $u(x, t)$ in $\mathrm{m} \mathrm{s}^{-1}$ as a function of the distance $x(\mathrm{~m})(\mathrm{a}-\mathrm{b})$ and of the solid phase $v(x, t)$ in $\mathrm{m} \mathrm{s}^{-1}$ as a function of the distance $x(\mathrm{~m})(\mathrm{c}-\mathrm{d})$ at different times for the collapse of a rectangular granular mixture over an inclined layer $\left(\theta=10^{\circ}\right)$ made of the same mixture. The initial volume fraction is $\varphi_{0}=0.6$. Here the friction law is the Richardson and Zaki drag force (RZ) with $m=1$.

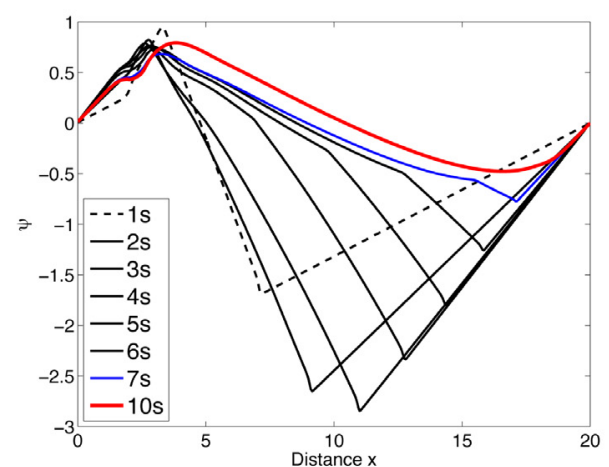

(a) $\psi$.

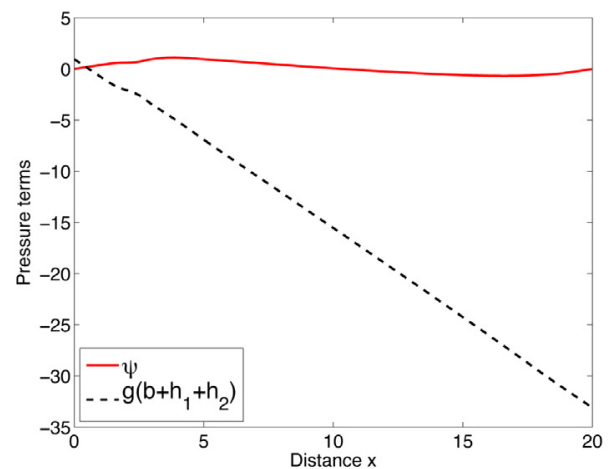

(b) $\psi$ and $b\left(g+h_{1}+h_{2}\right)$.

Figure 23. Test 2: left: the surface pressure of the solid phase $\psi(x, t)$ in $\mathrm{m}^{2} \mathrm{~s}^{-2}$ as a function of the distance $x(\mathrm{~m})$ at different times for the collapse of a rectangular granular mixture over an inclined layer $\left(\theta=10^{\circ}\right)$ made of the same mixture. Right: comparison between $\psi$ and $g\left(b+h_{1}+h_{2}\right)$ at $t=10 \mathrm{~s}$. 
Acknowledgements. We thank Farhang Radjai, Renaud Toussaint and Olivier Pouliquen for interesting discussions during this work. We thank Nicolas Mangold and Oldrich Hungr for leading the field trips in which we took the pictures in Figure 1. The work of E.D. Fernández-Nieto and G. Narbona-Reina was partially supported by the Spanish Government and FEDER through the Research projects MTM2009-07719 and MTM2012-38383-C02-02 and by the Andalusian Government through the project P11- RNM7069. Anne Mangeney and Francois Bouchut were funded by the ANR PLANETEROS and LANDQUAKES programmes.

\section{REFERENCES}

[1] T.B. Anderson and R. Jackson, A fluid mechanical description of fluidized beds. Ind. Eng. Chem. Fundam. 6 (1967) 527-539.

[2] K. Anderson, S. Sundaresan and R. Jackson, Instabilities and the formation of bubbles in fluidized beds. J. Fluid Mech. 303 (1995) 327-366.

[3] T. Aste, Circle, sphere, and drop packings. Phys. Rev. E 53 (1996) 2571.

[4] F. Boyer, O. Pouliquen and E. Guazzelli, Dense suspensions in rotating-rod flows: normal stresses and particle migration. J. Fluid Mech. 686 (2011) 5-25.

[5] F. Bouchut and M. Westdickenberg, Gravity driven shallow water models for arbitrary topography. Commun. Math. Sci. 2 (2004) 359-389.

[6] F. Bouchut, A. Mangeney-Castelnau, B. Perthame and J.-P. Vilotte, A new model of Saint Venant and Savage-Hutter type for gravity driven shallow water flows. C.R. Acad. Sci. Paris, Sér. I 336 (2003) 531-536.

[7] F. Bouchut, E. Fernandez-Nieto, A. Mangeney and P.-Y. Lagrée, On new erosion models of Savage-Hutter type for avalanches. Acta Mech. 199 (2008) 181-208.

[8] M. Farin, A. Mangeney and O. Roche, Dynamics, deposit and erosion processes in granular collapse over sloping beds. J. Geophys. Res. 119 (2013) 504-532.

[9] P. Favreau, A. Mangeney, A. Lucas, G. Crosta and F. Bouchut, Numerical modeling of landquakes. Geophys. Res. Lett. 37 (2010) L15305.

[10] D.L. George and R.M. Iverson, A two-phase debris-flow model that includes coupled evolution of volume fractions, granular dilatancy, and pore-fluid pressure. Italian J. Engrg. Geol. Environ. (2011) DOI:10.4408/IJEGE.2011-03.B-047.

[11] M. Goodman and S. Cowin, Two problems in the gravity flow of granular materials. J. Fluid Mech. 45 (1971) $321-339$.

[12] J. Happel and H. Brenner, Low Reynolds Number Hydrodynamics. Kluwer Academic Publishers (1983).

[13] H.J. Herrmann, R. Mahmoodi Baram and M. Wackenhut, Searching for the perfect packing. Phys. A 330 (2003) $77-82$.

[14] O. Hungr and S.G. Evans, Entrainment of debris in rock avalanches: An analysis of a long run-out mechanism. Bull. Geol. Soc. Am. 116 (2004) 1240-1252.

[15] R.M. Iverson, The physics of debris flows, Rev. Geophys. 35 (1997) 245-296.

[16] R.M. Iverson, R.P. Denlinger, Flow of variably fluidized granular masses across three-dimensional terrain 1: Coulomb mixture theory. J. Geophys. Res. 106 (2001) 537-552.

[17] R.M. Iverson, M. Logan, R.G. LaHusen and M. Berti, The perfect debris flow? aggregated results from 28 large-scale experiments. J. Geophys. Res. 115 (2010) F03005. DOI:10.1029/2009JF001514.

[18] R.M. Iverson, M.E. Reid, M. Logan, R.G. LaHusen, J.W. Godt and J.P. Griswold, Positive feedback and momentum growth during debris-flow entrainment of wet bed sediment. Nature Geoscience 4 (2011) 116-121.

[19] R. Jackson, The Dynamics of Fluidized Particles. Cambridges Monographs on Mechanics (2000).

[20] G. Jhonson, M. Massoudi and K.R. Rajagopal, A review of interaction mechanisms in fluidsolid flows. Technical Report. DOE/PETC/TR-90/9, U.S. Dep. of Energy, Pittsburgh Energy Tech. Ctr., Pittsburgh, USA (1990).

[21] F. Legros, The mobility of long-runout landslides. Eng. Geol. 63 (2002) 301-331.

[22] A. Lucas and A. Mangeney, Mobility and topographic effects for large Valles Marineris landslides on Mars. Geophys. Res. Lett. 34 (2007) L10201.

[23] A. Lucas, A. Mangeney and J.P. Ampuero, Frictional weakening in landslides on Earth and on other planetary bodies. Nature Commun. 5 (2014) 3417.

[24] A. Mangeney-Castelnau, J.P. Vilotte, M.O. Bristeau, B. Perthame, F. Bouchut, C. Simeoni and S. Yernini, Numerical modeling of avalanches based on Saint-Venant equations using a kinetic scheme. J. Geophys. Res. 108 (2003) 2527.

[25] A. Mangeney-Castelnau, F. Bouchut, J.P. Vilotte, E. Lajeunesse, A. Aubertin and M. Pirulli, On the use of Saint-Venant equations for simulating the spreading of a granular mass. J. Geophys. Res. 110 (2005) B09103.

[26] A. Mangeney, L. S. Tsimring, D. Volfson, I.S. Aranson and F. Bouchut, Avalanche mobility induced by the presence of an erodible bed and associated entrainment. Geophys. Res. Lett. 34 (2007) L22401.

[27] A. Mangeney, O. Roche, O. Hungr, N. Mangold, G. Faccanoni and A. Lucas, Erosion and mobility in granular collapse over sloping beds. J. Geophys. Res.-Earth Surf. 115 (2010) F03040.

[28] A. Mangeney, Landslide boost from entrainment. Nature Geosci. 4 (2011) 77-78.

[29] N. Mangold, A. Mangeney, V. Migeon, V. Ansan, A. Lucas, D. Baratoux and F. Bouchut, Sinuous gullies on Mars: Frequency, distribution, and implications for flow properties. J. Geophys. Res. Planets 115 (2010) E11001.

[30] C. Meruane, A. Tamburrino, O. Roche, On the role of the ambient fluid on gravitational granular flow dynamics. J. Fluid. Mech. 648 (2010) 381-404. 
[31] L. Moretti, A. Mangeney, Y. Capdeville, E. Stutzmann, C. Christian Huggel, D. Schneider and F. Francois Bouchut, Numerical modeling of the Mount Steller landslide flow history and of the generated long period seismic waves. Geophys. Res. Lett. 39 (2012) L16402.

[32] M.J. Niebling, E.G. Flekkoy, K.J. Mâloy and R. Toussaint, Mixing of a granular layer falling through a fluid. Phys. Rev. E $82(2010) 011301$.

[33] M. Ouriemi, P. Aussillous and E. Guazzelli, Sediment dynamics. Part I: Bed-load transport by shearing flows. J. Fluid Mech. 636 (2009) 295-319.

[34] C. Parés and M.J. Castro, On the well-balance property of Roe's method for nonconservative hyperbolic systems. Applications to shallow-water systems. ESAIM: M2AN 38 (2004) 821-852.

[35] M. Pailha and O. Pouliquen, A two-phase flow description of the initiation of underwater granular avalanches. J. Fluid Mech. 633 (2009) 115-135.

[36] M. Pelanti, F. Bouchut and A. Mangeney, A Roe-type scheme for two-phase shallow granular flows over variable topography. ESAIM: M2AN 42 (2008) 851-885.

[37] M. Pelanti, F. Bouchut and A. Mangeney, A Riemann solver for single-phase and two-phase shallow flow models based on relaxation. Relations with Roe and VFRoe solvers. J. Comput. Phys. 230 (2011) 515-550.

[38] E.B. Pitman and L. Le, A two-fluid model for avalanche and debris flows. Philos. Trans. R. Soc. A 363 (2005) $1573-1601$.

[39] S.P. Pudasaini, Y. Wang and K. Hutter, Modelling debris flows down general channels. Natural Hazards Earth System Sci. 5 (2005) 799-819.

[40] L. Rondon, O. Pouliquen and P. Aussillous, Granular collapse in a fluid: role of the initial volume fraction. Phys. Fluids 23 (2011) 073301.

[41] J.F. Richardson and W.N. Zaki, Sedimentation and Fluuidization: part I. Trans. Inst. Chem. Eng. 32 (1954) 35-53.

[42] O. Roche, M. Attali, A. Mangeney and A. Lucas, On the run out distance of geophysical gravitational flows: insight from fluidized granular collapse experiments. Earth Planet. Sci. Lett. 311 (2011) 375-385.

[43] O. Roche, Y. Niño, A. Mangeney, B. Brand, N. Pollock and G.A. Valentine, Dynamic pore pressure variations induce substrate erosion by pyroclastic flows, Geology 41 (2013) 1107-1110.

[44] S. Roux and F. Radjai, Texture-dependent rigid plastic behavior. In Proc. of Physics of Dry Granular Media 1997. Edited by H.J. Herrmann. Kluwer. Cargèse, France (1998) 305-311.

[45] S.B. Savage and K. Hutter, The motion of a finite mass of granular material down a rough incline. J. Fluid Mach. 199 (1989) $177-215$.

[46] U.E. Shamy and M. Zhegal, Coupled continuum discrete model for saturated granular materials. J. Engrg. Mech. 131 (2005) $413-426$.

[47] V. Topin, F. Dubois, Y. Monerie, F. Perales, A. Wachs: Micro-rheology of dense particulate flows: application to immersed avalanches. J. Non-Newtonian Fluid 166 (2011) 63-72.

[48] C. Voivret, F. Radjai and J.Y. Delenne, M.S. El Youssoufi, Space-filling properties of polydisperse granular media. Phys. Rev. E 76 (2007) 021301. 